\title{
Towards 20,20-difluorinated bryostatin: synthesis and biological evaluation of C17,C27-fragments
}

DOI:

10.1039/C8OB03152E

Document Version

Accepted author manuscript

Link to publication record in Manchester Research Explorer

\section{Citation for published version (APA):}

Mears, P. R., Hoekman, S., Rye, C. E., Bailey, F. P., Byrne, D. P., Eyers, P. A., \& Thomas, E. J. (2019). Towards 20,20-difluorinated bryostatin: synthesis and biological evaluation of C17,C27-fragments. Organic \& biomolecular chemistry, 17(6), 1487-1505. https://doi.org/10.1039/C8OB03152E

\section{Published in:}

Organic \& biomolecular chemistry

\section{Citing this paper}

Please note that where the full-text provided on Manchester Research Explorer is the Author Accepted Manuscript or Proof version this may differ from the final Published version. If citing, it is advised that you check and use the publisher's definitive version.

\section{General rights}

Copyright and moral rights for the publications made accessible in the Research Explorer are retained by the authors and/or other copyright owners and it is a condition of accessing publications that users recognise and abide by the legal requirements associated with these rights.

\section{Takedown policy}

If you believe that this document breaches copyright please refer to the University of Manchester's Takedown Procedures [http://man.ac.uk/04Y6Bo] or contact uml.scholarlycommunications@manchester.ac.uk providing relevant details, so we can investigate your claim.

\section{OPEN ACCESS}




\title{
Journal Name
}

\section{ARTICLE}

\section{Towards 20,20-difluorinated bryostatin}

z-CampillosReceived 00th January 20xx,

Accepted 00th January 20xx

DOI: $10.1039 / \times 0 \times x 00000 x$

www.rsc.org/

\section{Paul R. Mears ${ }^{a}$, Steven Hoekman ${ }^{a}$, Claire E. Rye, ${ }^{a}$ Fiona P. Bailey, ${ }^{b}$ Dominic P. Byrne, ${ }^{b}$ Patrick A.} Eyers $^{\mathrm{b}}$ and Eric J. Thomas ${ }^{\mathrm{a}^{*}}$

\begin{abstract}
Bryostatins with modified C17-C27 fragments have not been widely studied. The synthesis of 20,20-difluorinated analogues was therefore investigated. Such substitution would inhibit dehydration involving the C19-hydroxyl group and stabilise the ring-closed hemiacetal tautomers. Following preliminary studies, allyldifluorination was used to prepare difluorinated alkenols. Oxidation followed by stereoselective Wittig reactions of the resulting $\alpha, \alpha$-difluorinated ketones gave $(E)$ - $\alpha, \beta$-unsaturated esters that were taken through to complete syntheses of the 20,20-difluorinated C17-C27 fragments of bryostatin. These compounds showed modest binding to PKCs. Attempts were also undertaken to synthesise macrocyclic 20,20-difluorinated analogues. During preliminary studies, allyldifluorination was carried out using a 2-alkyl-3bromo-1,1-difluoropropene.
\end{abstract}

\section{Introduction}

Bryostatins, see bryostatin 1 (1) and 10 (2) in Fig. 1, are complex marine macrolides that have been intensively investigated because of their potent biological activities. ${ }^{1}$ The anticancer activity of bryostatin 1 in particular has been thoroughly studied and other useful clinical indications have been established. ${ }^{2}$
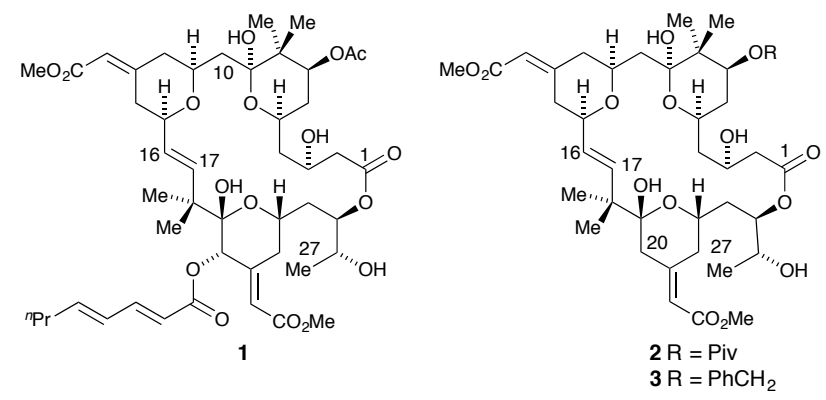

Fig. 1 Structures of representative bryostatins

Unfortunately the isolation of bryostatin from natural sources is still very challenging and so the chemical synthesis of bryostatins has been of considerable interest. ${ }^{3}$ Many approaches to their synthesis have been reported in the literature over the years ${ }^{4}$ and several total syntheses have now been completed. ${ }^{5}$ These equate to outstanding contributions to the chemistry of natural products and highlight the very latest techniques and methodogies of organic synthesis. The early

\footnotetext{
a. The School of Chemistry, The University of Manchester, Manchester, M13 9PL, $U K ;{ }^{b}$ The Department of Biochemistry, IIB, The University of Liverpool, Liverpool, L69 7ZB, UK.

* Corresponding author; e-mail: e.j.thomas@manchester.ac.uk.

Electronic Supplementary Information (ESI) includes copies of all the ${ }^{1} \mathrm{H},{ }^{13} \mathrm{C}$ and ${ }^{19} \mathrm{~F}$ NMR spectra reported in this paper. See DOI: $10.1039 / x 0 \times x 00000 x$
}

syntheses of these complex targets were substantial achievements in themselves, but they involved many steps with lengthy linear routes, and did not really deliver useful quantities of bryostatins. Recently, however, this situation has changed with a synthesis of bryostatin 1 on a multigramme scale. ${ }^{6}$ This development may meet clinical needs and illustrates the power of modern synthetic organic chemistry.

Bryostatins are believed to effect tumour inhibition by binding to the regulatory $\mathrm{C} 1$ domain of protein kinase $\mathrm{C}$ isotherms (PKCs). Interestingly phorbol esters bind to the same binding site of PKCs yet are tumour promoters. ${ }^{7}$ From molecular modelling studies using diacylglycerols (the natural PKC ligands), bryostatin 1 and phorbol esters, Wender postulated that the binding of bryostatin to PKCs involves the C1-carbonyl group together with the C19 and C26 hydroxyl groups with the C3 hydroxyl group helping to maintain the required conformation by forming an intramolecular hydrogen bond. ${ }^{8}$ This analysis led to the synthesis of macrocyclic analogues of bryostatins that possessed the $\mathrm{C} 17-\mathrm{C} 27$ fragment of bryostatin 1 so retaining the nanomolar binding to PKCs of the natural products. ${ }^{9,10}$ It was then discovered that analogues with different C1-C15 fragments could act as either tumour inhibitors or a tumour promoters so mimicking either bryostatin or the phorbol esters. ${ }^{10}$ Our work in this area culminated in a synthesis of the 7-benzyl ether $\mathbf{3}$ corresponding to bryostatin $10 \mathbf{2}$, the first synthesis of a bryostatin with no oxygenation at $\mathrm{C} 20{ }^{11}$

Most of the synthetic analogues with nanomolar binding are complex macrolides with $\mathrm{C} 17-\mathrm{C} 27$ fragments corresponding to those found in the natural products. Despite this feature that leads to potent PKC binding, we chose to investigate the synthesis of analogues of bryostatins in which the structure of the C17-C27 fragment had been modified to see whether any beneficial affects could accrue. One objective was to see whether simpler, non-macrocyclic, compounds could be prepared that retained the potent activity of bryostatins although whether such compounds would be tumour inhibitors 
or tumour promoters was difficult to predict. In particular, it was decided to study the synthesis of bryostatins in which two fluorine substituents had been introduced at C20, for example see structure 4 in Fig. 2. These compounds would retain the structural features that are important for binding, i.e the C1 carbonyl group and the hydroxyl groups at C19 and 26. However the electron withdrawing C20 fluorine substituents would help to stabilise the ring-closed hemi-acetal tautomer. They would also make the 21-methoxycarbonylmethylene group more electrophilic and lead to an increase in the pKa of the anomeric C19 hydroxyl group by about three pKa units. Perhaps most importantly, they would prevent dehydration, i.e. deactivation, involving loss of the C19 hydroxyl group. Fluorinated 2hydroxytetrahydropyrans have been prepared as surrogates of monosaccharides, ${ }^{12,13}$ and analogues of bryostatin with fluorinated B-rings have been synthesized for NMR studies. ${ }^{14}$

We here report full details on our work in this area and preliminary biological data. Some aspects of this work were disclosed in preliminary communications. ${ }^{15,16}$

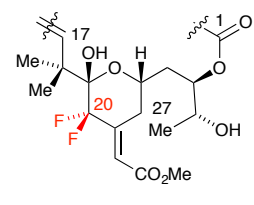

Fig. 2. The 20,20-difluorinated C17-C27 fragment of a bryostatin

\section{Results and discussion}

\section{Preliminary investigations}

Hydroxyketones 6 are the open-chain tautomers of the fluorinated bryostatin C-ring analogues $\mathbf{5}$. The first approach to these compounds was based on elaboration of the $\beta$ hydroxyesters 7 prepared from Reformatsky reactions ${ }^{17,18}$ of ethyl bromodifluoroacetate as shown in Fig. 3.

The Reformatsky reaction of 3-(2-mercatobenzothiazolyl)2,2-dimethylpropanal (8) with ethyl bromodifluoroacetate 11 using activated copper dust gave the hydroxyester 12 . This was protected as its triethylsilyl ether $\mathbf{1 3}$ using triethylsilyl trifluoromethanesulfonate and the ester converted into the corresponding methylketone $\mathbf{1 8}$ directly using methyllithium at $-78{ }^{\circ} \mathrm{C}$, Scheme 1. An excess of methyllithium at $\mathrm{rt}$ gave the corresponding tertiary alcohol (see experimental).

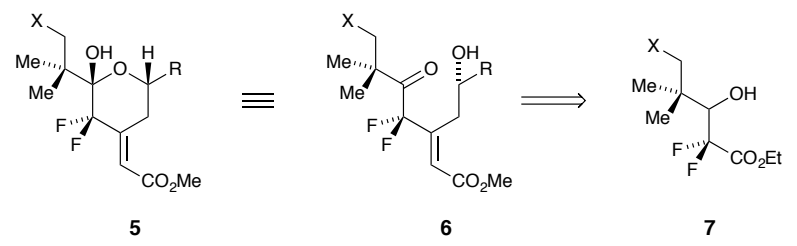

Fig. 3 Outline of the first approach to difluorinated intermediates

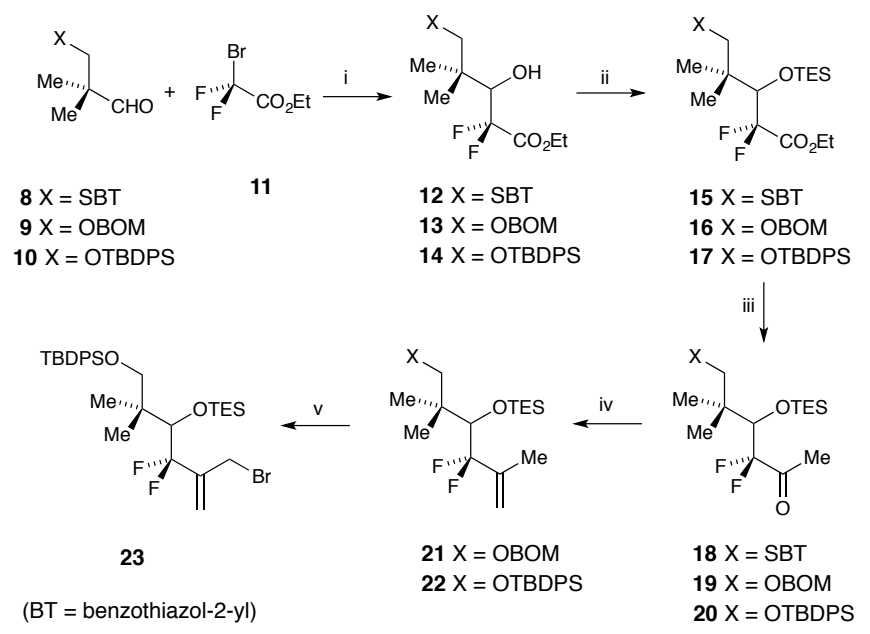

Scheme 1 Use of intermediates prepared by Reformatsky reactions of ethyl bromodifluoroacetate Reagents and conditions i, Zn powder, $\mathrm{CuCl}, \mathrm{THF}, \mathrm{rt}, 30 \mathrm{~min}$, add 11, $10 \mathrm{~min}$, add the aldehyde in THF, heat under reflux, 16-36 h (12, 83\%; 13, 75\%; 14, 78\%); ii, 2,6-lut., TESOTf, DCM, rt, 1-10 d (15, 75\%; 16, 85\%; 17, 94\%); iii, MeLi, THF, $-78{ }^{\circ} \mathrm{C}, 5.5$ h (18, 77\%; 19, $86 \%$; 20, 55\%); iv, $\mathrm{Ph}_{3} \mathrm{PMeBr}$, KHMDS, tol., $0{ }^{\circ} \mathrm{C}, 30 \mathrm{~min}$, add the ketone, rt, $48 \mathrm{~h}(\mathbf{2 1}, 75 \% ; 22,73 \%) ; \mathrm{v}, \mathrm{NBS}, \mathrm{CHCl}_{3}, \mathrm{hv}, 10 \mathrm{~min}$, heat under reflux $24 \mathrm{~h}(23,<72 \%)$.

The intention was to develop the methyl ketone 18 to introduce the required side-chain, see proposed intermediate 6 . However, an attempted aldol condensation with $(E)$ crotonaldehyde gave unchanged starting material and an attempted Wittig reaction to convert the ketone into the corresponding alkene was also unsuccessful. Since these difficulties may have been due to the 2-mercaptobenzothiazole, 3-(benzyloxymethoxy)-2,2-dimethylpropanal (9) was taken through to the methylketone 19 . The Wittig reaction with methylene(triphenyl)phosphorane now gave a good yield of the alkene $\mathbf{2 1}$ and the same sequence was successful starting with the 3-tert-butyldiphenylsilyloxypropanal $10^{19,20}$ giving the alkene 22. This was then converted into the allylic bromide $\mathbf{2 3}$ under free-radical conditions although on scale-up this reaction was somewhat capricious. The intention had been to use a reaction of the allylic bromide $\mathbf{2 3}$ with an aldehyde to assemble intermediates analogous to the target compounds 6 . Although modifications to this synthesis could be envisaged, the relative inaccessability of bromide $\mathbf{2 3}$ led to this approach being discontinued, see Scheme 1.

Copper catalysed coupling reactions of bromo- and iododifluoroacetates with aryl and alkenyl iodides have been reported. ${ }^{21,22}$ This chemistry could lead to a flexible synthesis of the unsaturated difluoroesters $\mathbf{2 4}$ from the alkenyl iodides 26, see Fig. 4. Ozonolysis followed by an (E)-selective HornerWadsworth-Emmons reaction would introduce the required $(E)$ methoxycarbonylmethylene group. Modification of the ethyl ester should provide the required ketone. Indeed HornerWadsworth-Emmons reactions of $\alpha, \alpha$-difluorinated ketones are known to be $(E)$-selective. ${ }^{23}$ 


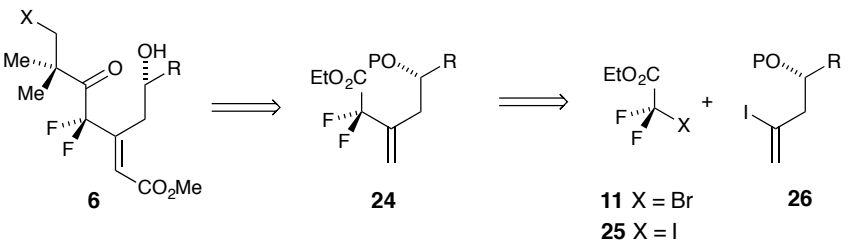

Fig. 4 The proposed use of unsaturated difluorinated esters

To access the intermediate $\mathbf{2 4}$ where $\mathrm{R}=$ ethyl, the alkenyl iodide $\mathbf{2 9}$ was prepared from the alkyne $\mathbf{2 7}$ by addition of hydrogen iodide generated in $\operatorname{situ}^{24}$ followed by silylation. However, in our hands, the copper catalysed coupling of this with the iododifluoroester $\mathbf{2 5}$ gave only a low yield of the difluorinated alkene $\mathbf{3 0}$. The analogous coupling of the alkenyl iodides $\mathbf{3 1}$ and $\mathbf{3 2}$ with the iododifluoroester $\mathbf{2 5}$ also gave low yields of the products 33 and $\mathbf{3 4}$, Scheme 2, and so this approach was not taken any further.

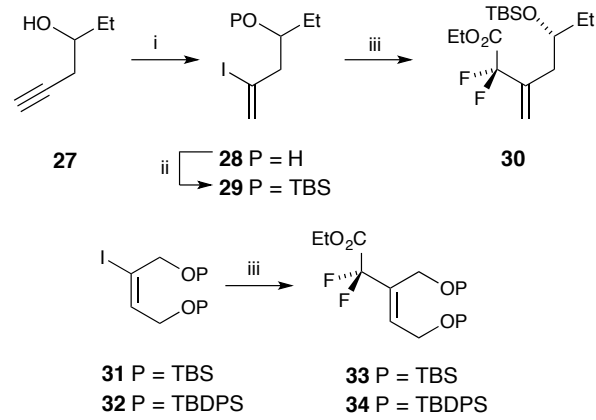

Scheme 2 Attempted coupling of alkenyl iodides and ethyl iododifluoroacetate Reagents and conditions i, Nal, TMSCl, $\mathrm{MeCN}, \mathrm{H}_{2} \mathrm{O}$, rt, 20 min, add 27, rt, 4 h (80\%); ii, TBSCl, imid., DCM, rt, 24 h (75\%); iii, 25, Cu, DMSO, rt, 3 h, add alkenyl iodide, DMSO, rt, 1.25 h (30, 8\%; 33, 12\%; 34, 14\%).

Allyldifluorination is well known for the introduction of geminal difluorine containing fragments ${ }^{25}$ and so the homoallylic difluoroalcohols $\mathbf{3 6}$ should be readily accessible from the bromodifluoropropene $\mathbf{3 7}$ and aldehydes $\mathbf{3 8}$ as shown in Fig. 5. Oxidation and an (E)-selective Horner-WadsworthEmmons reaction would then provide the unsaturated esters $\mathbf{3 5}$ that could be converted into the hydroxyketones 6 by selective modification of the terminal double bond.

The indium powder mediated reaction ${ }^{25}$ of 3-bromo-3,3difluoropropene (37) with 3-tert-butyldiphenylsilyloxypropanal (39) gave a good yield of the difluorinated alkene $\mathbf{4 0}$ that was protected as the bis-silyl ether $\mathbf{4 1}$, see Scheme 3. Ozonolysis and

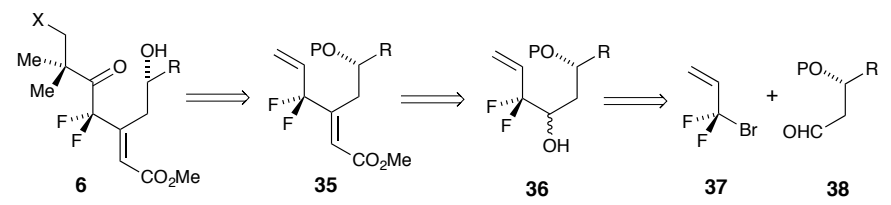

Fig. 5 Use of difluoroallylation in a synthesis of the hydroxyketones 6

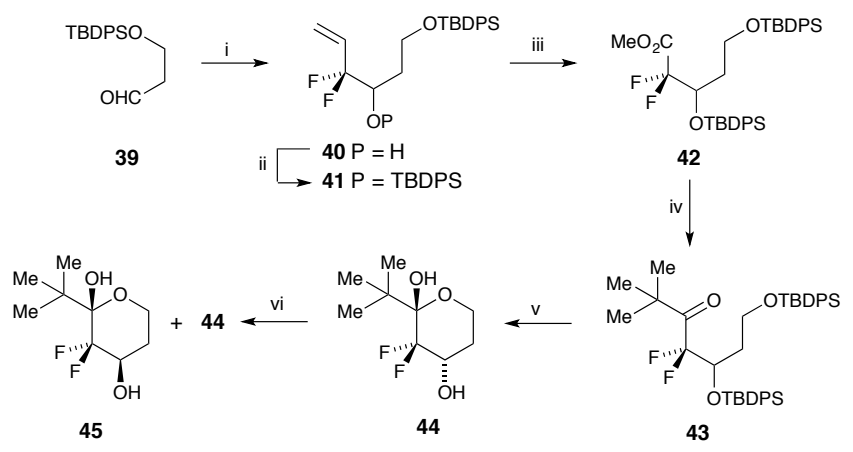

Scheme 3 Preparation of the 2-hydroxytetrahydropyran 44 Reagents and conditions i, In powder, Lil, $\mathrm{H}_{2} \mathrm{O}, 37, \mathrm{rt}, 10 \mathrm{~min}$, add 39, THF, rt, 5 h (97\%); ii, crude 40, TBDPSCl, imid., DCM, rt, $48 \mathrm{~h}$ (72\% from 37); iii, (a) $\mathrm{O}_{3}, \mathrm{DCM},-78{ }^{\circ} \mathrm{C}, 5 \mathrm{~min}$, add DMS, rt, $3 \mathrm{~h}$ (b) ${ }^{t} \mathrm{BuOH}, 2$-methylbut-2-ene, THF, $\mathrm{NaClO}_{2}, \mathrm{Na}_{2} \mathrm{H}_{2} \mathrm{PO}_{4} \cdot 2 \mathrm{H}_{2} \mathrm{O}$, rt, 16 h (c) $\mathrm{TMSCHN}_{2}, \mathrm{MeOH}$, tol., rt, $48 \mathrm{~h}$ (80\% from 41); iv, ${ }^{t}$ BuLi, pentane, THF, $-78{ }^{\circ} \mathrm{C}, 5 \mathrm{~h}(52 \%) ; \mathrm{v}, \mathrm{TBAF}, \mathrm{THF}, 0{ }^{\circ} \mathrm{C}$ to rt, $15 \mathrm{~h}\left(72 \%\right.$ after cryst.); vi, $\mathrm{C}_{6} \mathrm{D}_{6}, \mathrm{rt}, 48 \mathrm{~h}(44: 45=40: 60)$.

further oxidation of the resulting aldehyde gave the corresponding acid that was converted into the ester $\mathbf{4 2}$ using trimethylsilyldiazomethane. The conversion of ester $\mathbf{4 2}$ into the ketone $\mathbf{4 3}$ was achieved in one step using tert-butyllithium ${ }^{26}$ and desilylation using tetrabutylammonium fluoride gave a mixture of two inseparable compounds. On cooling a single product crystallised out from this mixture and was identified as the 3,3difluoro-2,4-dihydroxytetrahydropyran $\mathbf{4 4}$ using $X$-ray crystallography, see Fig. 6 . On standing in solution in deuteriated chloroform (not base-washed), this hemiacetal gave a 40:60 mixture in favour of the second component after $10 \mathrm{~min}$ at rt. In benzene- $d_{6}$ or in base-washed chloroform, the equilibration took place more slowly but gave a similar mixture of the two compounds, typically within $48 \mathrm{~h}$. Attempts to isolate the second component were unsuccessful as the initially isolated compound $\mathbf{4 4}$ tended to crystallise out. However, the IR spectrum of a mixture did not show a carbonyl peak and the ${ }^{1} \mathrm{H}$ NMR spectrum of the mixture was consistent with the second component being the epimer 45. It would appear that the two hemiacetals equilibrate despite the presence of the geminal fluorine substituents. However, the open chain, hydroxyketone tautomer was not a significant component of the mixture at equilibrium. In the crystalline tautomer $\mathbf{4 4}$ the tert-butyl and 4hydroxyl groups are equatorial whereas in the slightly more stable epimer $\mathbf{4 5}$, both hydroxyl groups are axial.

The reaction of prenylmagnesium chloride with the ester $\mathbf{4 3}$ gave the ketone $\mathbf{4 6}$ that on deprotection gave a mixture of products from which the 2,4-dihydroxytetrahydropyran 47 was isolated by repeated trituration, see Scheme 4 . As before in

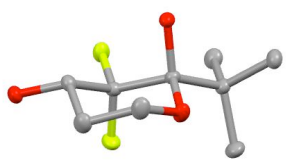

Fig. 6 The structure of the crystalline dihydroxytetrahydropyran 44 as established by X-ray crystallography. 

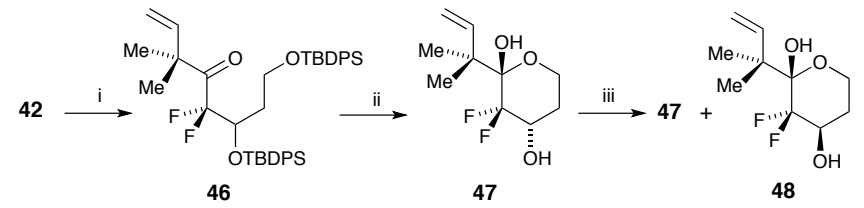

Scheme 4 Synthesis of the 2-hydroxytetrahydropyran 47 Reagents and conditions i, 3-methylbut-2-enylmagnesium chloride, THF, $-10{ }^{\circ} \mathrm{C}, 12 \mathrm{~h}(66 \%) ; \mathrm{ii}, \mathrm{TBAF}, \mathrm{THF}, 0{ }^{\circ} \mathrm{C}$ to $\mathrm{rt}, 15 \mathrm{~h}$ (82\% afer repeated trituration); iii, $\mathrm{C}_{6} \mathrm{D}_{6}, \mathrm{rt}, 48 \mathrm{~h}(47: 48=60: 40)$.

benzene- $d_{6}$, this product was found to equilibrate with a second component identified as the epimeric hemiacetal 48 . In this case, 47:48 $=60: 40$, at equilibrium. Structures were assigned to these products by analogy with the 2,4hydroxytetrahydropyrans $\mathbf{4 4}$ and $\mathbf{4 5}$. In both cases the doublets attributed to the fluorines were significantly more widely separated in their ${ }^{19} \mathrm{~F}$ NMR spectra for the crystalline epimers 44 and $\mathbf{4 7}$ (see experimental).

It was recognised that 2-substituted 3-bromo-3,3difluoropropenes 49 could react with aldehydes to give difluoroalkenes 50, see Fig. 7, and that these might be incorporated into C17-C27 fragments of bryostatin more easily than the less functionalised intermediates 36, cf. Fig. 5. 2Substituted 3-bromo-3,3-difluoropropenes have been used in palladium-catalysed reactions with aryl boronic acids ${ }^{27}$ but their indium mediated reactions with aldehydes have not been investigated.

1-Chloro-1,1-difluorodecan-2-one (52) was prepared from from methyl chlorodifluoroacetate (51) according to the literature procedure. ${ }^{28}$ Conversion into the epoxide $\mathbf{5 3}$ was then achieved using di-iodomethane and methyllithium ${ }^{29}$ and the epoxide was taken through to the allylic alcohol $\mathbf{5 4}$ by treatment with tert-butyllithium. This allylic alcohol was converted into 2bromomethyl-1,1-difluorodecene $\mathbf{5 5}$ using $N$-bromosuccinimide and dimethyl sulfide. On heating this allylic bromide in toluene under reflux, isomerisation gave the required 2(bromodifluoromethyl)decene $\mathbf{5 6}$ but only as a mixture with the starting bromide, $\mathbf{5 5}: \mathbf{5 6}=\mathbf{2 0}: \mathbf{8 0}$, see Scheme 5 . The indium powder mediated reaction of this mixture of allylic bromides with benzaldehyde gave a modest, $50 \%$, yield of the required adduct 57a. However, better yields were obtained using the bromide $\mathbf{5 5}$ and the yield of $\mathbf{5 7 a}$ improved to $84 \%$ in DMF. Indeed the allylic bromide $\mathbf{5 5}$ reacted under these conditions with a range of aldehydes to give products $\mathbf{5 7}$ in reasonable to excellent yields, see Scheme $5 .^{16}$

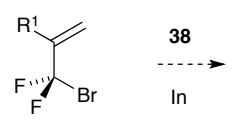

49

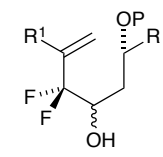

50
Fig. 7 Posssible reactions of 2-substituted 1-bromo-1,1difluoropropenes with aldehydes.

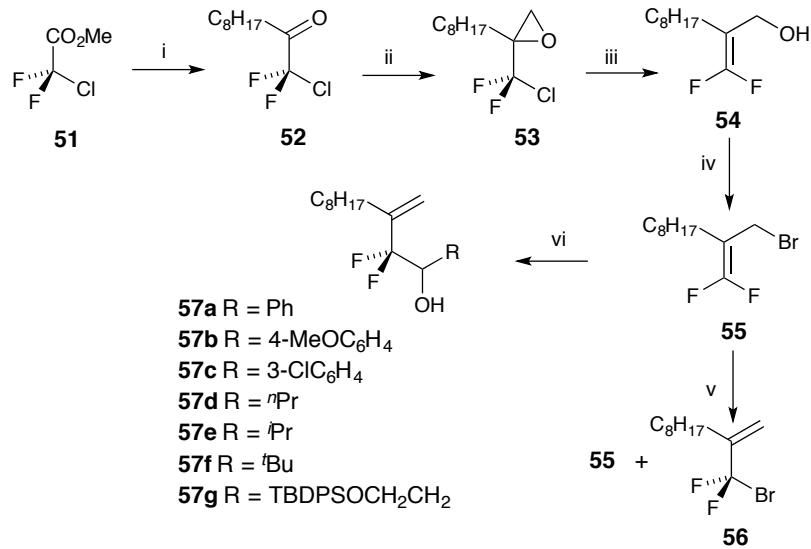

Scheme 5 Chemistry of 2-bromomethyl-1,1-difluorodecene 55 Reagents and conditions i, $\mathrm{C}_{8} \mathrm{H}_{17} \mathrm{MgBr}, \mathrm{Et}_{2} \mathrm{O}$, tol., $-78{ }^{\circ} \mathrm{C}$ to -20 ${ }^{\circ} \mathrm{C}, 10 \mathrm{~min}$ (96\%); ii, $\mathrm{CH}_{2} \mathrm{I}_{2}$, MeLi, THF, $-78{ }^{\circ} \mathrm{C}$ to rt; iii, ${ }^{t} \mathrm{BuLi}$, pentane, THF, $-78{ }^{\circ} \mathrm{C}$ to $\mathrm{rt}, 16 \mathrm{~h}$ (44\% from 52); iv, NBS, $\mathrm{Me}_{2} \mathrm{~S}$, $\mathrm{DCM}, 0{ }^{\circ} \mathrm{C}, 10 \mathrm{~min}$, cool to $-20{ }^{\circ} \mathrm{C}$, add 54, rt, $15 \mathrm{~h} \mathrm{(63 \% );} \mathrm{v,} \mathrm{tol.,}$ heat under reflux, $15 \mathrm{~h}$ (55:56 = 20:80, 89\%); vi, In, DMF, rt, 10 min, add aldehyde, rt, 14 h (57a, 84\%; 57b, 65\%; 57c, 93\%; 57d, 98\%; 57e, 68\%; 57f, 47\%; 57g, 91\%).

Generally Wittig ${ }^{30}$ and Horner-Wadsworth-Emmons reactions ${ }^{23}$ of $\alpha, \alpha$-difluorinated ketones give $\alpha, \beta$-unsaturated esters with useful (E)-stereoselectivity. However, before embarking on a synthesis of a fluorinated C17-C27 fragment of bryostatin, it was decided to check the stereoselectivity of the introduction of the methoxycarbonylmethylene unit in our system, $c f$. Fig. 5 .

Oxidation of the previously prepared alcohols $\mathbf{4 0}$ and $\mathbf{5 7 g}$ gave the ketones $\mathbf{5 8}$ and $\mathbf{6 1}$. The ketoester $\mathbf{6 3}$ was prepared by oxidation of the alcohol prepared by the samarium di-iodide mediated Reformatsky reaction of ethyl bromodifluoroacetate (11) and the protected hydroxypropanal $39 .{ }^{31}$ The Wittig reactions of the ketones $\mathbf{5 8}$ and $\mathbf{6 3}$ with the stabilised ylid, methoxycarbonylmethylene(triphenyl)phosphorane, were usefully $(E)$-selective, $>85: 15$, with the $(E)$-ester 62 being the only product isolated from ketone $\mathbf{6 1}$, see Scheme 6 .

The $(E)$-configuration was assigned to the major products of these reactions using NMR. For example, the vinylic protons in the major and minor products of the reaction with ketone $\mathbf{5 8}$ were at $\delta 6.21$ and $\delta 5.98$, respectively, consistent with the vinylic hydrogen being closer to the fluorine substituents in the major isomer. For the minor product, significant nOe enhancement of a 5-hydrogen was observed on irradiation of the vinylic proton consistent with this being the $(Z)$-isomer $\mathbf{6 0}$. The fluorines in the major and minor products were at $\delta$ -100.52 and $\delta-95.73$ consistent with the literature ${ }^{23,30}$ for analogous difluorinated $(E)$ - and $(Z)$ - $\alpha, \beta$-unsaturated esters. Similar data confirmed the $(E)$-configuration of the major products $\mathbf{6 2}$ and $\mathbf{6 4}$ from the Wittig reactions of ketones $\mathbf{6 1}$ and 63 (see experimental). 


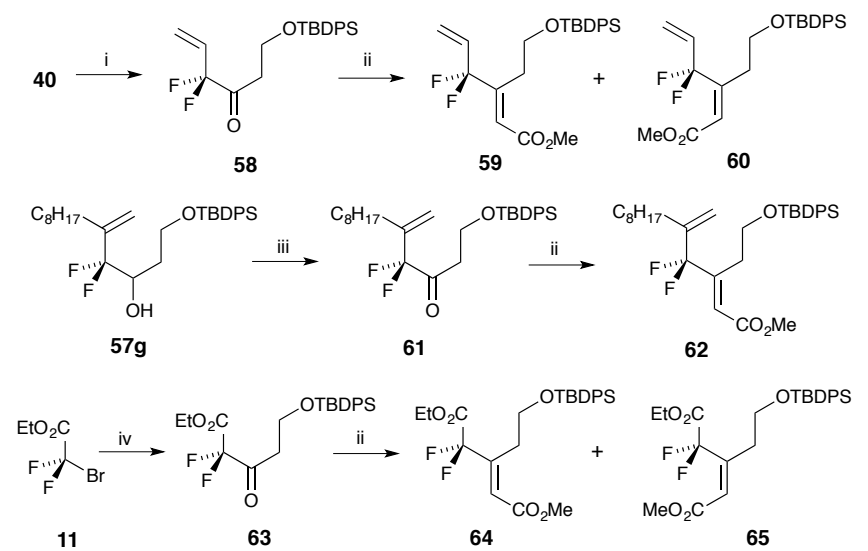

Scheme $6(E)$-Selective Wittig reactions of $\alpha, \alpha$-difluorinated ketones Reagents and conditions i, TPAP, NMO, 4Å sieves, DCM, rt, 16 h (78\%); ii, $\mathrm{Ph}_{3} \mathrm{P}=\mathrm{CHCO}_{2} \mathrm{Me}$, tol., 70-90 ${ }^{\circ} \mathrm{C}, 6-16 \mathrm{~h}$ (59, 84\%; 60, 11\%; 62, 48\%; 64, 82\%; 65, 12\%); iii, DMSO, $(\mathrm{COCl})_{2}$, DCM, $-78{ }^{\circ} \mathrm{C}, 30 \mathrm{~min}$, add $57 \mathrm{~g}, \mathrm{DCM},-78{ }^{\circ} \mathrm{C}, 45 \mathrm{~min}, \mathrm{Et} \mathrm{N}_{3}, \mathrm{rt}, 1 \mathrm{~h}$ (61\%); iv, (a) Sml $, 11,39$, THF, rt, $10 \mathrm{~min}$ (98\%) (b) TPAP, NMO, $4 \AA ̊$ sieves, DCM, rt, $16 \mathrm{~h}$ (63\%).

To incorporate the bis-ester 64 into a synthesis of a 2hydroxytetrahydropyran analogous to the hemiacetal $\mathbf{4 7}$ (see Scheme 4), it would be necessary to develop conditions for the regioselective reaction of prenylmagnesium bromide with the ethoxycarbonyl group. It was decided instead to attempt to prepare the corresponding aldehyde by the regioselective oxidative cleavage of the terminal double bond in the diene $\mathbf{5 9}$ as the selective cleavage of terminal double bonds in dienes using osmium tetroxide is well known. ${ }^{32}$ Although in the case of diene 59, the terminal double bond will be deactivated towards oxidation by the adjacent fluorine substituents, this is also true for the double bond conjugated to the ester. The dihydroxylation of alkenes with allylic fluorine substituents by osmium tetroxide is known. ${ }^{32}$

In our hands the dihydroxylation of the diene $\mathbf{5 9}$ using osmium tetroxide and $\mathrm{N}$-methylmorpholine- $\mathrm{N}$-oxide in wateracetone was very slow at $\mathrm{rt}$ and gave a low yield of diol 66 (29\%) together with unchanged starting material. Under more forcing conditions, the yield improved to $44 \%$ but side-products that appeared to have been formed by additional hydroxylation of the double bond conjugated to the ester were also detected. The use of ruthenium tetroxide generated in situ by the slow addition of sodium periodate to a solution of the alkene and ruthenium(III) chloride ${ }^{33}$ gave a $44 \%$ yield of the diol 66 together with unchanged starting material. This procedure was quite reliable and side products formed by hydroxylation of the unsaturated ester were not isolated, Scheme 7.

Cleavage of the diol 66 using lead(IV) acetate gave the aldehyde 67. Rather than react this with a Grignard reagent, the crude aldehyde was added to the prenyl bromide in THF and the mixture transferred to a suspension of zinc powder and titanocene dichloride in THF. ${ }^{34}$ This procedure gave a reasonable yield of the alcohol 68 that was oxidised to the ketone 69 . Desilyation using HF.pyridine then gave the required 2hydroxytetrahydropyran $\mathbf{7 0}$, Scheme 7.

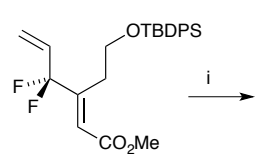

59
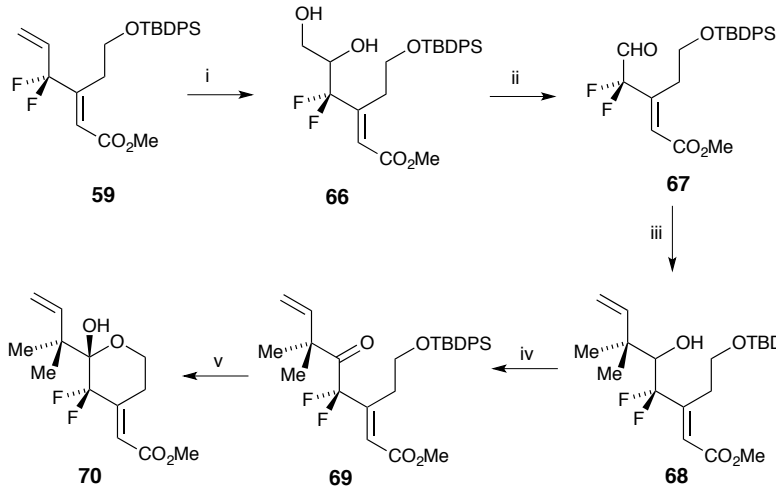

67

iii

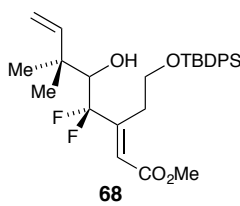

Scheme 7 Synthesis of the 3,3-difluoro-2-hydroxy-4-[(E)methoxycarbonylmethylene]tetrahydropyran 70 Reagents and conditions i, $\mathrm{RuCl}_{3} . \mathrm{H}_{2} \mathrm{O}$, EtOAc, $\mathrm{MeCN}, \mathrm{H}_{2} \mathrm{O}$, add $\mathrm{NalO}_{4}$ over $5 \mathrm{~h}$, rt, $39 \min \left(66,44 \%\right.$, recovered 59, 44\%); ii, $\mathrm{Pb}(\mathrm{OAc})_{4}, \mathrm{NaHCO}_{3}$, DCM, rt, $30 \mathrm{~min}$; iii, $\mathrm{Me}_{2} \mathrm{C}=\mathrm{CHCH}_{2} \mathrm{Br}$, THF, add to $\mathrm{Zn}$ powder, titanocene dichloride (cat.), THF, rt, 2.5 h (65\% from 66); iv,

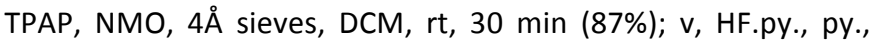
THF, rt, $2.5 \mathrm{~h}$ (82\%).

Structures were assigned to the intermediates in Scheme 7 from spectroscopic data and by analogy with earlier work. The hemiacetal carbon was observed as a double-doublet at $\delta 99.1$ in its proton decoupled ${ }^{13} \mathrm{C}$ NMR spectrum due to slightly different coupling constants $\left({ }^{2} J_{\mathrm{C}-\mathrm{F}} 30.1,24.4 \mathrm{~Hz}\right)$ with the axial and equatorial fluorines.

This synthesis had given the 3,3-difluoro-2-hydroxy-4-[(E)methoxycarbonylmethylene]tetrahydropyran $\mathbf{7 0}$ in eight steps from 3-tert-butyldimethylsilylpropanal 39. Although the hydroxylation of the alkene $\mathbf{5 9}$ was not particularly efficient, it was decided to use the chemistry outlined in Scheme 7 to synthesise a fully substituted C17-C27 fragment of a bryostatin, cf. structure 4 in Fig. 2.

Synthesis and chemistry of 2-hydroxy-3,3difluorotetrahydropyrans analogous to a difluorinated C17-C27 fragment of bryostatin

The known alkene $\mathbf{7 1 ^ { 3 5 }}$ was was ozonolysed with reduction of the intermediate ozonide using triphenylphosphine to give the aldehyde 72. The indium-mediated difluoroallylation of this aldehyde gave a mixture of the epimers of alcohol 73. Samples of these were separated for characterisation and the mixture was oxidised to give the ketone $\mathbf{7 4}$. The Wittig reaction of this ketone with methoxycarbonylmethylene(triphenyl)phosphorane was highly stereoselective and gave the required $(E)$-alkene $\mathbf{7 5}$ albeit with a small amount of unchanged starting material. Regioselective hydroxylation of the diene $\mathbf{7 5}$ was carried out using ruthenium tetroxide, and the resulting diol $\mathbf{7 6}$ was taken through to the alcohol 77, as a mixture of epimers, by cleavage using lead(IV) acetate and reaction of the resulting aldehyde with prenyl bromide mediated by zinc powder and titanocene dichloride. Oxidation of the alcohol 77 then gave the ketone 78, Scheme 8. 


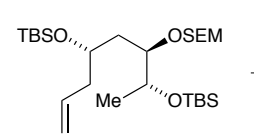

71

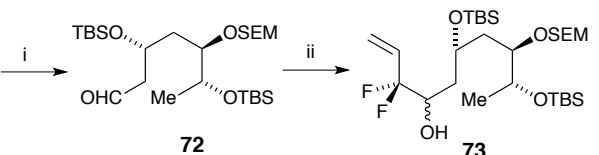

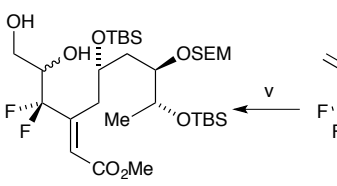

76

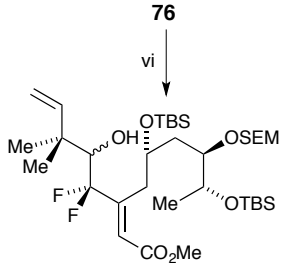

77

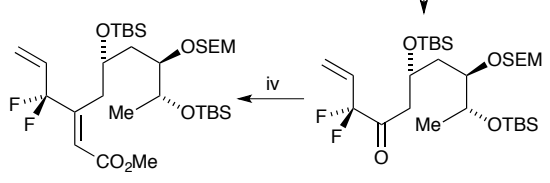

75

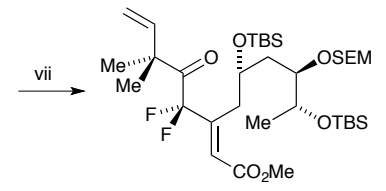

78
Scheme 8 Synthesis of ketone 78 Reagents and conditions $\mathrm{i}, \mathrm{O}_{3}$, DCM, $-78{ }^{\circ} \mathrm{C}, 5 \mathrm{~min}, \mathrm{Ph}_{3} \mathrm{P}, \mathrm{rt}, 3 \mathrm{~h}(96 \%)$; ii, In powder, 37, rt, $4 \mathrm{~h}$ (66\%, 53:47); iii, TPAP, NMO, DCM, $4 \AA$ sieves, rt, 16 h (75\%); iv, $\mathrm{Ph}_{3} \mathrm{P}=\mathrm{CHCO}_{2} \mathrm{Me}$, tol., $90{ }^{\circ} \mathrm{C}, 16 \mathrm{~h}$ (75, 64\%; 74, 10\%), v, 75, $\mathrm{RuCl}_{3} . \mathrm{H}_{2} \mathrm{O}, \mathrm{EtOAC}, \mathrm{MeCN}, \mathrm{H}_{2} \mathrm{O}$, add $\mathrm{NaIO}_{4}$ over $9 \mathrm{~h}(76,55: 45$, 61\%; 75, 39\%); vi, (a) $\mathrm{NaHCO}_{3}, \mathrm{~Pb}(\mathrm{OAc})_{4}, \mathrm{DCM}, \mathrm{rt}, 90$ min (b) crude aldehyde and $\mathrm{Me}_{2} \mathrm{C}=\mathrm{CHCH}_{2} \mathrm{Br}$, THF added to $\mathrm{Zn}$ dust, titanocene dichloride (cat.), THF, rt, 16 h (77, 50:50, 70\%); vii, TPAP, NMO, DCM, rt, $16 \mathrm{~h}$ (60\%).

Selective removal of the 2-(trimethylsilylethoxy)methoxy (SEM) group from the protected hydroxyketone $\mathbf{7 8}$ gave the alcohol 79 that was acylated to give the butanoyl and isobutanoyl esters $\mathbf{8 0}$ and $\mathbf{8 1}$. Desilylation with in situ acetal formation gave mixtures of the esters $\mathbf{8 4 / 8 6}$ and $\mathbf{8 5 / 8 7}$ formed by equilibration of the regioisomers under the reaction conditions, Scheme 9. Selective removal of the tertbutyldimethylsilyl groups from the fully protected hydroxyketone $\mathbf{7 8}$ gave the hemiacetal 82. Removal of the SEMgroup then gave the corresponding diol 83 .

Structures were assigned to the products in Scheme 9 from spectroscopic data. The hemiacetals 82-87 were single compounds $\left({ }^{1} \mathrm{H}\right.$ and $\left.{ }^{13} \mathrm{C} N M R\right)$ with their configurations at $\mathrm{C} 2$ assigned on the basis of the anomeric effect. Spin-decoupling and 2D NMR methods were used to distinguish between the different regioisomers (see experimental).

To establish the effect of the $\mathrm{C} 20$ geminal fluorine substituents on the PKC binding and biological activity of analogues of bryostatin, it was decided to synthesise a fluorinated macrolide closely related to a non-fluorinated compound that had already been prepared. Amongst the simplest macrocyclic compounds that are known to show potent PKC binding are the salicylic esters $\mathbf{8 8}$ prepared by Wender. ${ }^{36,37}$ It was therefore decided to prepare a close analogue of this system and the macrodiolide $\mathbf{8 9}$ was identified as a suitable target, Fig. 8.

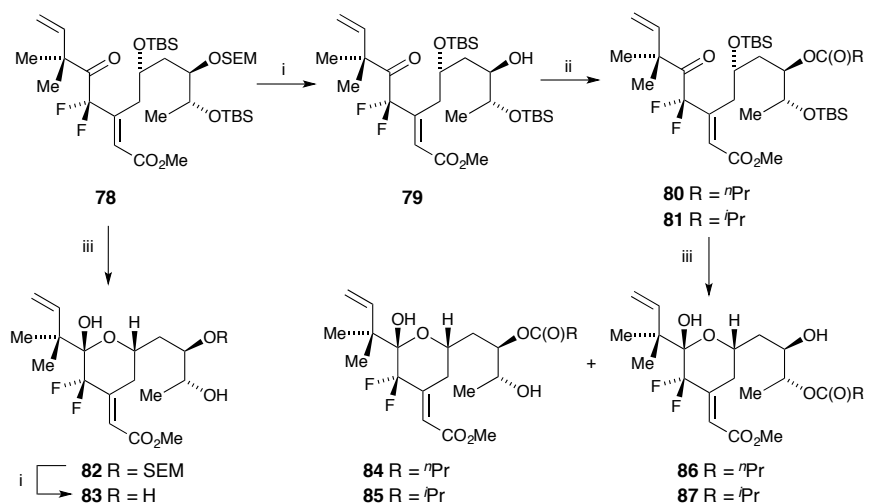

Scheme 9 Synthesis of C20 difluorinated analogues of the C17C27 fragment of bryostatin Reagents and conditions i, $\mathrm{MgBr}_{2}$, $\mathrm{Et}_{2} \mathrm{O}, \mathrm{MeNO}_{2}, \mathrm{rt}, 2-24 \mathrm{~h}(79,74 \% ; 83,70 \%) ; \mathrm{ii}, \mathrm{RCO}_{2} \mathrm{H}, 2,4,6-$ $\mathrm{Cl}_{3} \mathrm{C}_{6} \mathrm{H}_{2} \mathrm{COCl}, \mathrm{Et}_{3} \mathrm{~N}, \mathrm{rt}, 1.5 \mathrm{~h}$, add 79, DMAP, tol., rt, $16 \mathrm{~h}(80$,

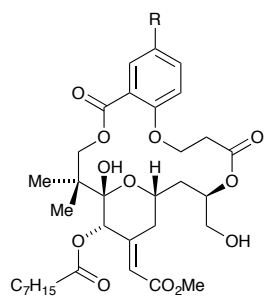

88

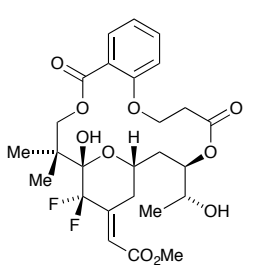

89
$80 \%$; 81, 94\%); iii, HF.py., py., THF, $0{ }^{\circ} \mathrm{C}, 16$ h (82, 67\%; 84, 42\%; $86,42 \% ; 85,23 \% ; 87,23 \%)$.

Fig. 8. Simple macrocyclic analogues of bryostatin.

Regioselective hydroxylation of the diene $\mathbf{7 7}$ followed by oxidative cleavage of the resulting vicinal diol gave the aldehyde 90. Reduction using sodium borohydride gave the diol $\mathbf{9 1}$ as a mixture of epimers. Regioselective esterification of this diol using the protected salicylic acid $\mathbf{9 8}$ gave the ester $\mathbf{9 2}$ that was oxidised to give the ketoester 93. Removal of the SEM ether under standard conditions gave the alcohol $\mathbf{9 4}$ and cleavage of this 2,2,2-trichloroethyl ester gave the seco-acid 95. However, an attempted macrocyclisation of this hydroxyacid did not give the required macrodiolide. Instead the major product appeared to have a terminal double bond and was provisionally identified from ${ }^{1} \mathrm{H}$ NMR data as the acrylate $\mathbf{9 6}$ formed by an elimination of the salicylate under the reaction conditions. In Wender's syntheses of the macrodiolides $\mathbf{8 8},^{36}$ the assembly of the macrocycle was carried out on intermediates that already had the C-19 hemiacetal functionality intact. In our case, the hemiacetal was not present in the seco-acid 95 . This meant that the transannular hydrogen bonding found in bryostatins was not able to facilitate macrocyclisation. Unfortunately further studies were not possible and a synthesis of a macrocyclic analogue of a 20,20-difluorinated bryostatin was not completed, see Scheme 10. 


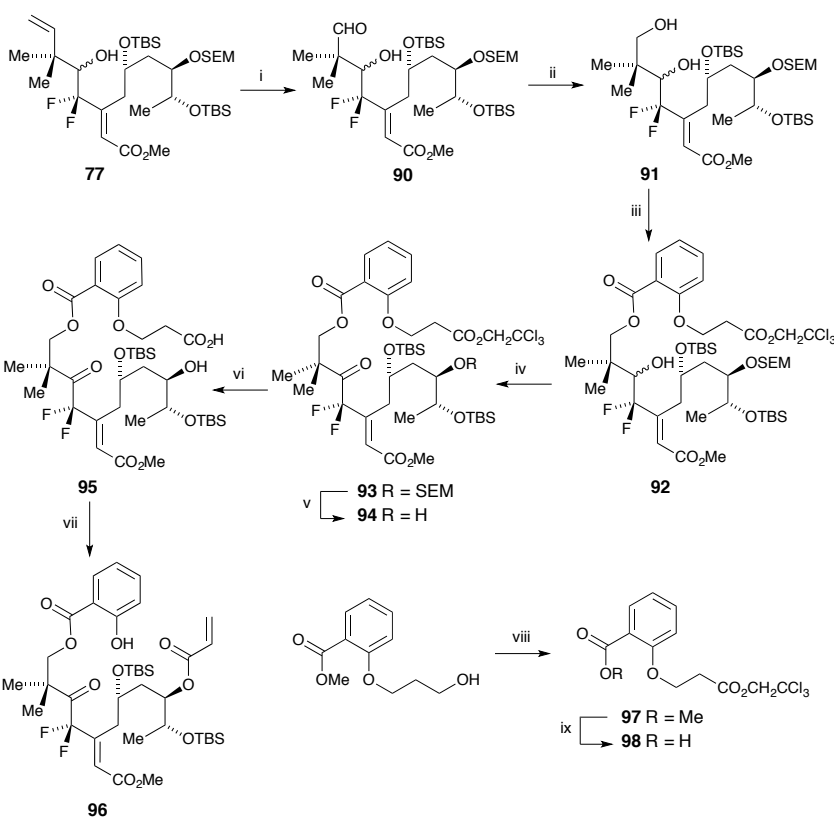

Scheme 10 Approaches to the macrodiolide 89 Reagents and conditions i, (a) $\mathrm{OsO}_{4}, \mathrm{NMO},{ }^{t} \mathrm{BuOH}, \mathrm{H}_{2} \mathrm{O}, \mathrm{rt}, 4 \mathrm{~h}$ (b) $\mathrm{Pb}(\mathrm{OAc})_{4}$, $\mathrm{Na}_{2} \mathrm{CO}_{3}, \mathrm{DCM}$, rt, 5 min (82\% from 77); ii, $\mathrm{NaBH}_{4}, \mathrm{MeOH}, 0{ }^{\circ} \mathrm{C}, 1$ h (83\%); iii, 98, 2,4,6- $\mathrm{Cl}_{3} \mathrm{C}_{6} \mathrm{H}_{2} \mathrm{COCl}, \mathrm{Et}_{3} \mathrm{~N}$, tol., rt, $1.5 \mathrm{~h}$, add 91, DMAP, tol., rt, $16 \mathrm{~h}$; iv, TPAP, NMO, 4Å sieves, DCM, rt, $16 \mathrm{~h}$ (57\% from 91); v, $\mathrm{MgBr}_{2}, \mathrm{MeCN} \mathrm{Et}_{2} \mathrm{O}, \mathrm{rt}, 15 \mathrm{~h}$ (65\%); vi, $\mathrm{Zn}$, PPTS, THF, reflux, 30 min; vii, 2,4,6- $\mathrm{C}_{6} \mathrm{H}_{2} \mathrm{COCl}$, py., benzene, rt, $45 \mathrm{~min}$, add to DMAP, benzene, $60{ }^{\circ} \mathrm{C}, 16 \mathrm{~h}$; viii, (a) TEMPO, $\mathrm{Phl}(\mathrm{OAC})_{2}, \mathrm{MeCN}, \mathrm{H}_{2} \mathrm{O}, \mathrm{rt}, 1 \mathrm{~h}$ (b) 2-methyl-but-2-ene, $\mathrm{NaH}_{2} \mathrm{PO}_{4}$, $\mathrm{NaClO}_{2}, 0{ }^{\circ} \mathrm{C}, 1 \mathrm{~h}$ (c) $\mathrm{Cl}_{3} \mathrm{CCH}_{2} \mathrm{OH}, \mathrm{DCC}$, DMAP, DCM, rt, $16 \mathrm{~h}$ (73\%); ix, Nal, $\mathrm{TMSCl}, \mathrm{DCE}, 100{ }^{\circ} \mathrm{C}, 15 \mathrm{~h}(72 \%)$.

\section{PKC Binding studies}

Preliminary studies were next carried out into binding of the fluorinated hemiacetals $\mathbf{8 3}, \mathbf{8 4}$ and $\mathbf{8 6}$ to purified PKC proteins. Phorbol esters and bryostatins target individual cysteinerich zinc finger motifs ( $\mathrm{C} 1$ and $\mathrm{C} 2$ domains) of conventional and novel PKCs, and are believed to lead to allosteric release of the pseudosubstrate region. Thermal profiling has been used to evaluate atypical PKC isozyme destabilisation by small molecules ${ }^{38}$ and upon binding, nanomolar to micromolar affinity kinase inhibitors usually induce temperature shifts in the range of $2-10{ }^{\circ} \mathrm{C}$ in vitro. ${ }^{39,40}$ In order to analyse whether bryostatin binding to PKC isozymes can also be evaluated in a thermal shift assay, Sypro Orange, a fluorophore exhibiting enhanced fluorescence when it binds to unfolded protein kinases, ${ }^{40,41}$ was employed for real-time thermal profiling with three affinity-tagged purified kinases, namely $P K C \alpha$, representative of the conventional PKCs, and a known phorbol ester and bryostatin target, ${ }^{42} \mathrm{PKC} \xi$, an 'atypical' PKC protein that lacks canonical $\mathrm{C} 1$ and $\mathrm{C} 2$ domains, and PKA, the archetypal Ser/Thr protein kinase from the 'AGC family' to which PKC also belongs. As shown in Fig. 9A, all three kinase preparations were essentially pure, making

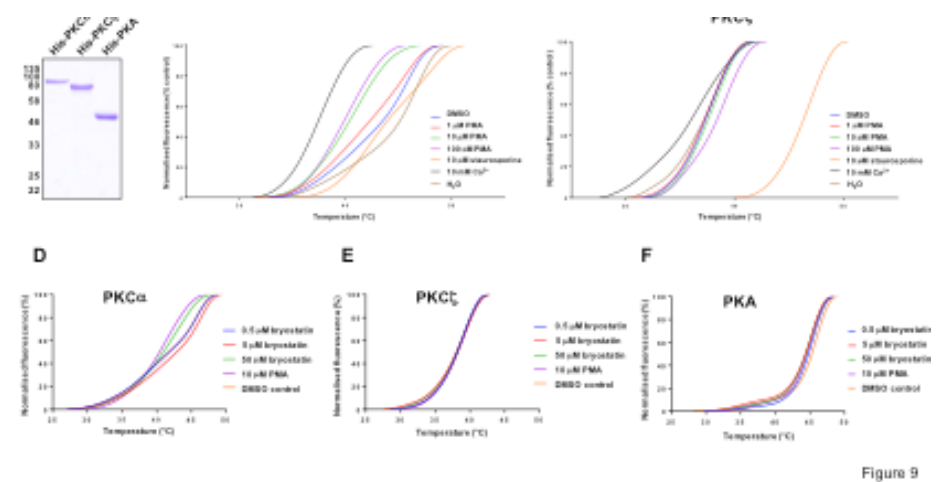

Fig. 9. A novel fluorescence-based PKC thermal shift assay. (A) Analysis of $\mathrm{N}$-terminally $6 \mathrm{His}$ affinity-tagged human $\mathrm{PKC} \alpha$ (amino acids 1-672), human PKC $\zeta$ (amino acids 2-592) and fulllength murine PKA (1-351). $2 \mathrm{mg}$ of the indicated purified proteins were separated by SDS-PAGE and stained with Coomassie Blue. The positions of a range of molecular mass markers are also shown (kDa). (B) Differential scanning fluorimetry reveals PKC $\alpha$ unfolding profiles as a function of temperature in the presence of a series of different compounds. (C) PKC $\zeta$ unfolding profiles evaluated as a function of temperature in the presence of the indicated compounds. Thermal profiling of each kinase in the presence of a range of concentrations of pure commercial bryostatin was assessed using purified (D) PKC $\alpha,(E)$ PKC $\zeta$, or (F) PKA catalytic subunit. The final concentration of each kinase in the assays was $5 \mu \mathrm{M}$. Triplicate data were fitted to the Boltzmann Equation, and are shown for duplicate experiments. DMSO was the control solvent, and purified phorbol ester (PMA) was the positive control compound employed for PKC $\alpha$. Similar results were seen in an independent experiment.

them suited to real-time thermal stability studies in the presence and absence of chemical compounds.

PKC $\alpha$ underwent a complex unfolding transition that was influenced markedly in the presence of PMA, which induced a $3^{\circ} \mathrm{C}$ destabilisation relative to control, consistent with polypeptide domain structural reorganisation. This destabilising effect was even more marked with $\mathrm{Ca}^{2+}$ ions, leading to a $>6^{\circ} \mathrm{C}$ change in thermal stability relative to control. In contrast, the broad PKC ATP-binding site inhibitor staurosporine ${ }^{44}$ promoted an expected PKC $\alpha$ stabilisation, consistent with interaction with the nucleotide-binding (ATP) site. Based on thermal profiling, little or no destabilisation by PMA was detected for PKC $\zeta$, consistent with absence of a $\mathrm{C} 2$ domain. In contrast, $\mathrm{Ca}^{2+}$ ions markedly destabilised $\mathrm{PKC} \zeta$, which possesses an atypical $\mathrm{Ca}^{2+}$ binding C1 domain, and staurosporine induced a $+9^{\circ} \mathrm{C}$ stabilisation, in line with its reported nanomolar potency towards this PKC isozyme. ${ }^{43}$ We next evaluated the effect of commercial byrostatin on each PKC isozyme, and included PKA, which lacks $\mathrm{C} 1$ or $\mathrm{C} 2$ domains, as an additional negative control. As shown in Figure 9D, bryostatin induced robust destabilisation of PKC $\alpha$ at 10 -fold molar compound excess, similar to PMA, but had no detectable effect on PKC $\zeta$ or PKA. To our knowledge, this is the first time that a thermal shift-assay has been employed to demonstrate bryostatin interaction with PKC $\alpha$. We next 

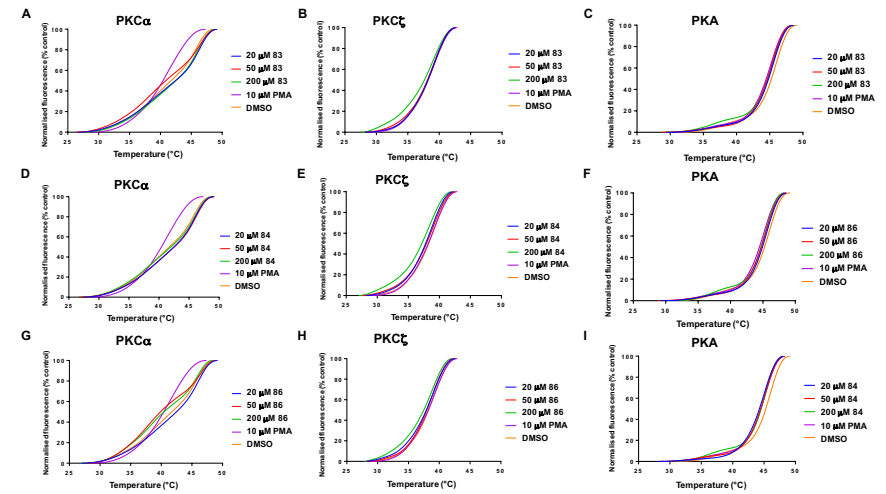

Fig. 10. PKC Thermal shift analysis of fluorinated bryostatins. Real-time thermal stability assay for each kinase was performed in the presence and absence of the indicated concentration of bryostatin analogues 83 (A-C), 84 (D-F) or 86 (G-I). The final concentration of each purified protein kinase was $5 \mu \mathrm{M}$. Triplicate data were fitted to the Boltzmann Equation, and are shown for duplicate experiments. DMSO was the control solvent, and PMA was the positive control compound for PKC $\alpha$. Similar results were seen in independent experiments.

evaluated the effects of compounds 83,84 and 86 on temperature-dependent PKC unfolding. As detailed in Fig. 10, 83had a very subtle destabilising effect on $P K C \alpha$, but was without effect on $\mathrm{PKC} \zeta$ or PKA, suggestive of a weak interaction. In marked contrast, $\mathbf{8 6}$ induced a complex, but dose-dependent bryostatin-like destabilisation of PKC $\alpha$, but not PKC $\zeta$ or PKA, suggesting direct binding to a biologically relevant $\mathrm{PKC} \alpha$ conformation. No effects of $\mathbf{8 4}$ were noted on $\mathrm{PKC} \alpha, \mathrm{PKC} \zeta$ or PKA, even at 40 -fold molar excess compounds. Together, these data establish a new semi-quantitative assay for rapidly evaluating binding of bryostatins to PKC isozymes, and raise the likelihood that fluorinated compounds such as $\mathbf{8 6}$ might possess biological PKC $\alpha$ regulatory activity. Testing this hypothesis will be the focus of future experiments, which will include enzyme based inhibitor assays in-cell thermal stability assays (CETSA) employing endogenous PKC isozymes.

\section{Summary and conclusions}

This paper reports the synthesis and preliminary PKC binding studies of a series of hemiacetals that correspond to the C17C27 fragment of bryostatin. These compounds were selected for study since they retain the key structural features identified as being critical in the nanomolar binding of bryostatin to PKCs yet represent modified C17-C27 fragments that had not been evaluated previously. It was hoped that the two electronwithdrawing fluorine substituents at $\mathrm{C} 20$ (bryostatin numbering) would stabilise the ring-closed, hemi-acetal tautomers and inhibit any dehydration involving the crucial C19 hydroxyl group. It was recognised that the fluorine substituents would also increase the acidity of the $\mathrm{C} 19$ hydroxyl group by about three
pKa units and make the C21 methylene substituent more electrophilic.

In the event, the fluorine containing hemi-acetals $82-87$ were prepared in $c a$. 10 steps from the known alkene $\mathbf{7 1}$. The key steps in these syntheses were the difluoroallylation of the aldehyde 72 and the $(E)$-selective Wittig reaction of the $\alpha, \alpha$ difluorinated ketone 74. During the course of the work other approaches were evaluated including the difluoroallylation using more the complex difluoroalkene 55. Attempts to complete a synthesis of a difluorinated macrocyclic analogue of a bryostatin could not be concluded because of issues that were encountered in the macrocyclisation of the seco-acid $\mathbf{9 5 .}$

The binding of the fluorinated compounds 83,84 and 86 with purified human PKC $\alpha$ were studied and very promising early data obtained based on comparative profiling with authentic bryostatin 1. It is hoped that this work will encourage other research workers to develop further analogues of bryostatins of the type presented in this paper with the objective of discovering relatively simple compounds that retain the potent biological activity of bryostatin.

\section{Experimental}

\section{General experimental details}

Flash column chromatography was performed using Merck silica gel $(60 \mathrm{H}, 230-300$ mesh). Base washed siica was prepared by stirring silica in saturated aqueous potassium hydrogen carbonate for $24 \mathrm{~h}$ then rinsing with deionised water until the washings were $\mathrm{pH} 7$, followed by rigorous drying in an oven.

Light petroleum refers to the fraction boiling between 40 and $60{ }^{\circ} \mathrm{C}$ and was redistilled. Tetrahydrofuran was dried over sodium-benzophenone and was distilled under nitrogen. Dichloromethane was dried over $\mathrm{CaH}_{2}$ and was distilled. Ether refers to diethyl ether. Reactions under non-aqueous conditions were carried out under an atmosphere of nitrogen or argon.

Mass spectra used electron impact ionisation $\left(\mathrm{EI}^{+}\right)$, chemical ionisation using ammonia $\left(\mathrm{Cl}^{+}\right)$, electrospray ionisation in the positive mode $\left(\mathrm{ES}^{+}\right)$, atmospheric pressure chemical ionisation in the positive mode $\left(\mathrm{APCl}^{+}\right)$and time of flight $\mathrm{MS}$ with electrospray ionisation (TOF $\left.\mathrm{ES}^{+}\right)$. Low resolution mass spectra were recorded on a Waters SQD2 or on an Agilent 5975C Triple axis spectrometer. High resolution mass spectra were recorded using a Thermo Finnigan MAT95XP or on a Waters QTOF spectrometer. Infra-red spectra were measured using a Bruker Alpha P FTIR spectrometer on $\mathrm{NaBr}$ plates, either neat or as evaporated films. Nuclear magnetic resonance spectra were recorded using Bruker Avance 300, Bruker Ultrashield 400 or on Bruker Ultrashield 500 spectrometers at $c a .25{ }^{\circ} \mathrm{C}$ unless otherwise stated. Coupling constants $(J)$ are given in Hertz $(\mathrm{Hz})$ and chemical shifts are relative to tetramethylsilane. Residual non-deuteriated solvent was used as the internal standard.

Differential scanning fluorimetry (DSF) was performed with a StepOnePlus Real-Time PCR machine (Life Technologies) using Sypro-Orange dye (Invitrogen) and thermal ramping $\left(0.3{ }^{\circ} \mathrm{C}\right.$ in step intervals between 25 and $94^{\circ} \mathrm{C}$ ). PKA catalytic subunit was 
purified as described previously. ${ }^{41}$ Purified PKC proteins were purchased from MRC-PPU Reagents and Services (University of Dundee), and were diluted to a final concentration of $5 \mu \mathrm{M}$ in 50 $\mathrm{mM}$ Tris/ $/ \mathrm{HCl}, \mathrm{pH} 7.4$ and $100 \mathrm{mM} \mathrm{NaCl}$ in the presence or absence of the indicated concentrations of ligand (final DMSO concentration was never higher than $4 \% \mathrm{v} / \mathrm{v})$. Normalized data were processed using the Boltzmann equation to generate sigmoidal denaturation curves, and these were plotted as previously described. ${ }^{44}$ Staurosporine (S-9300), bryostatin 1 (B6697) and phorbol 12-myristate 13-acetate (PMA, P-1680) were all purchased from LC Laboratories and dissolved and diluted in DMSO prior to use.

\section{3-(Benzothiazol-2-ylsulfanyl)-2,2-dimethylpropanal}

Triphenylphosphine (18 $\mathrm{g}, 68 \mathrm{mmol})$ and 2mercaptobenzothiazole $(11.4 \mathrm{~g}, 68 \mathrm{mmol})$ was added to neopentyl glycol $(10 \mathrm{~g}, 96 \mathrm{mmol})$ in THF $(150 \mathrm{~mL})$ and the solution cooled to $0{ }^{\circ} \mathrm{C}$. Di-isopropyl azodicarboxylate $(13.4 \mathrm{~mL})$ in THF $(200 \mathrm{~mL})$ was added dropwise over $1 \mathrm{~h}$ and the solution stirred at $0{ }^{\circ} \mathrm{C}$ for $2 \mathrm{~h}$ and at $\mathrm{rt}$ for $16 \mathrm{~h}$. Water $(200 \mathrm{~mL})$ was added followed by EtOAc $(200 \mathrm{~mL})$ and the aqueous layer was extracted with EtOAc $(3 \times 100 \mathrm{~mL})$. The organic extracts were washed with aqueous $\mathrm{NaOH}(1 \mathrm{M}, 100 \mathrm{~mL})$ and brine $(100 \mathrm{~mL})$, dried $\left(\mathrm{MgSO}_{4}\right)$ and concentrated under reduced pressure. Chromatography of the residue (90:10 light petroleum:EtOAc) gave 3-(benzothiazol-2-ylsulfanyl)-2,2-dimethylpropanol (14.5 g, $84 \%$ ) as a colourless oil, $R_{\mathrm{f}}=0.8$ (EtOAc) (Found: Found: $\mathrm{M}^{+}+$ $\mathrm{H}, 254.0667 . \mathrm{C}_{12} \mathrm{H}_{16} \mathrm{NOS}_{2}$ requires $\left.\mathrm{M}, 254.0668\right) ; \delta_{\mathrm{H}}(400 \mathrm{MHz}$, $\left.\mathrm{CDCl}_{3}\right) 0.99\left(6 \mathrm{H}, \mathrm{s}, 2 \times 2-\mathrm{CH}_{3}\right), 3.27$ and 3.31 (each $2 \mathrm{H}, \mathrm{s}, 1-\mathrm{H}_{2}$ or 3- $\mathrm{H}_{2}$ ), 5.10 (1 H, br. s, OH), $7.23(1 \mathrm{H}, \mathrm{td}, J$ 8.0, 1.6, ArH), 7.35 (1 $\mathrm{H}, \mathrm{td}, J \mathrm{~J} .6,1.6, \mathrm{ArH}$ ) and 7.66 and 7.73 (each $1 \mathrm{H}, \mathrm{d}, J$ 8.0, $\mathrm{ArH}$ ); $\delta_{\mathrm{C}}\left(100 \mathrm{MHz}, \mathrm{CDCl}_{3}\right) 24.2,37.5,42.1,67.8,120.9,121.0,124.6$, 126.3, 134.8, 152.0 and 169.9; $\mathrm{m} / z\left(\mathrm{ES}^{+}\right) 276\left(\mathrm{M}^{+}+23,100 \%\right)$ and $254\left(\mathrm{M}^{+}+1,32\right)$.

Dess-Martin periodinone $(2.5 \mathrm{~g}, 5.9 \mathrm{mmol})$ was added to this alcohol $(1.0 \mathrm{~g}, 3.95 \mathrm{mmol})$ in DCM $(20 \mathrm{~mL})$ and the mixture was stirred at $\mathrm{rt}$ for $16 \mathrm{~h}$. Saturated aqueous sodium bisulfite $(15 \mathrm{~mL})$ and saturated sodium bicarbonate $(15 \mathrm{~mL})$ were added and the mixture stirred until gas evolution stopped. The aqueous layer was extracted with DCM $(3 \times 20 \mathrm{~mL})$ and the organic extracts were dried $\left(\mathrm{MgSO}_{4}\right)$ and concentrated under reduced pressure. Chromatography of the residue (75:25 light petroleum:EtOAc) gave the title compound $\mathbf{8}(0.8 \mathrm{~g}, 81 \%)$ as a yellow oil, $R_{\mathrm{f}}=0.9$ (EtOAc) (Found: $\mathrm{M}^{+}+\mathrm{H}, 252.0516 . \mathrm{C}_{12} \mathrm{H}_{14} \mathrm{NOS}_{2}$ requires $\mathrm{M}$, 252.0512); $v_{\text {max }} / \mathrm{cm}^{-1} 2976,1774,1726,1460,1428,1241,1095$, 1017 and 757; $\delta_{\mathrm{H}}\left(400 \mathrm{MHz}, \mathrm{CDCl}_{3}\right) 1.18\left(6 \mathrm{H}, \mathrm{s}, 2 \times 2-\mathrm{CH}_{3}\right), 3.58$ $\left(2 \mathrm{H}, \mathrm{s}, 3-\mathrm{H}_{2}\right), 7.22$ and 7.33 (each $\left.1 \mathrm{H}, \mathrm{t}, J 8.0, \mathrm{ArH}\right), 7.67$ and 7.78 (each $1 \mathrm{H}, \mathrm{d}, J$ 8.0, $\mathrm{ArH}$ ) and $9.57(1 \mathrm{H}, \mathrm{s}, 1-\mathrm{H}) ; \delta_{\mathrm{c}}(100 \mathrm{MHz}$, $\mathrm{CDCl}_{3}$ ) 21.3, 31.0, 39.1, 121.1, 121.4, 124.4, 126.1, 135.3, 152.8, 166.7 and 203.7; $\mathrm{m} / \mathrm{z}\left(\mathrm{ES}^{+}\right) 274\left(\mathrm{M}^{+}+23,100 \%\right)$ and $252\left(\mathrm{M}^{+}+1\right.$, 52).

\section{3-(Benzyloxymethyloxy)-2,2-dimethylpropanal}

(9). Diisopropylethylamine $(3.9 \mathrm{~mL}, 22 \mathrm{mmol}$ ) and chloromethyl benzyl ether $(2.0 \mathrm{~mL}, 14 \mathrm{mmol})$ were added to neopentyl glycol $(3.0 \mathrm{~g}$, $29 \mathrm{mmol})$ in DCM $(30 \mathrm{~mL})$ and the solution was stirred at $\mathrm{rt}$ for $16 \mathrm{~h}$. Water $(20 \mathrm{~mL})$ was added and the aqueous layer was extracted with DCM $(3 \times 20 \mathrm{~mL})$. The organic extracts were dried $\left(\mathrm{MgSO}_{4}\right)$ and concentrated under reduced pressure. Chromatography of the residue (75:25 light petroleum:EtOAc) gave 3-benzyloxymethoxy-2,2-dimethylpropanol (2.5 g, $81 \%$ ) as a colourless oil, $R_{\mathrm{f}}=0.35$ (65:35 light petroleum:EtOAc) (Found: $\mathrm{M}^{+}+\mathrm{Na}, 247.1306 . \mathrm{C}_{13} \mathrm{H}_{20} \mathrm{O}_{3} \mathrm{Na}$ requires $\left.\mathrm{M}, 247.1305\right) ; v_{\max } / \mathrm{cm}^{-}$ 1 3367, 3029, 2879, 1578, 1496, 1457, 1308, 1207, 1041, 1023, 908 and $735 ; \delta_{\mathrm{H}}\left(400 \mathrm{MHz}, \mathrm{CDCl}_{3}\right) 0.95\left(6 \mathrm{H}, \mathrm{s}, 2 \times 2-\mathrm{CH}_{3}\right), 3.45$ and 3.47 (each $2 \mathrm{H}, \mathrm{s}, 1-\mathrm{H}_{2}$ or $\left.3-\mathrm{H}_{2}\right), 4.62\left(2 \mathrm{H}, \mathrm{s}, \mathrm{PhCH}_{2}\right), 4.76$ (2 $\left.\mathrm{H}, \mathrm{s}, \mathrm{OCH}_{2} \mathrm{O}\right)$ and 7.34-7.37 (5 H, m, ArH); $\delta_{\mathrm{C}}\left(125 \mathrm{MHz}, \mathrm{CDCl}_{3}\right)$ 21.9, 36.1, 69.6, 71.1, 76.5, 91.6, 127.8, 128.5 and 137.6; $\mathrm{m} / \mathrm{z}$ $\left(\mathrm{ES}^{+}\right) 247\left(\mathrm{M}^{+}+23,100 \%\right)$.

Molecular sieves (7.0 g), NMO (1.65 g, $14 \mathrm{mmol})$ and TPAP ( $82 \mathrm{mg}, 0.23 \mathrm{mmol}$ ) were added to this alcohol $(2.1 \mathrm{~g}, 9.4 \mathrm{mmol})$ in DCM $(20 \mathrm{~mL})$ and acetonitrile $(5 \mathrm{~mL})$ and the mixture stirred at $\mathrm{rt}$ for $2 \mathrm{~h}$ and then filtered through a pad of silica that was washed with DCM $(3 \times 30 \mathrm{~mL})$ and EtOAc $(3 \times 30 \mathrm{~mL})$. The filtrate and washings were concentrated under reduced pressure and chromatography of the residue the gave title compound 9 (1.52 g, $73 \%)$ as a colourless oil, $R_{\mathrm{f}}=0.6(65: 35$ light petroleum:EtOAc); $v_{\max } / \mathrm{cm}^{-1} 3032,2936,2877,1703,1474$, $1455,1157,1109,1044$ and $738 ; \delta_{\mathrm{H}}\left(400 \mathrm{MHz} \mathrm{CDCl}_{3}\right) 1.12(6 \mathrm{H}$, $\left.\mathrm{s}, 2 \times \mathrm{CH}_{3}\right), 3.61\left(2 \mathrm{H}, \mathrm{s}, 3-\mathrm{H}_{2}\right), 4.58\left(2 \mathrm{H}, \mathrm{s}, \mathrm{PhCH}_{2}\right), 4.74(2 \mathrm{H}, \mathrm{s}$, $\left.\mathrm{OCH}_{2} \mathrm{O}\right), 7.31-7.36(5 \mathrm{H}, \mathrm{m}, \mathrm{ArH})$ and $9.56(1 \mathrm{H}, \mathrm{s}, 1-\mathrm{H}) ; \delta_{\mathrm{c}}(100$ $\left.\mathrm{MHz}_{1} \mathrm{CDCl}_{3}\right)$ 19.0, 46.8, 69.5, 72.7, 94.8, 127.8, 127.9, 128.4, 137.6 and 205.0; $\mathrm{m} / \mathrm{z}\left(\mathrm{ES}^{+}\right) 245\left(\mathrm{M}^{+}+23,100 \%\right)$.

Ethyl 5-(2-benzothiazolylsulfanyl)-2,2-difluoro-3-hydroxy4,4-dimethylpentanoate (12). Acid washed zinc powder $(0.9 \mathrm{~g}$, $14 \mathrm{mmol}$ ) and copper(I) chloride (cat.) were added to THF (10 $\mathrm{mL}$ ) under nitrogen and the suspension stirred at $\mathrm{rt}$ for $30 \mathrm{~min}$. Ethyl bromodifluoroacetate $11(1.3 \mathrm{~mL}, 10 \mathrm{mmol})$ was added followed, after $10 \mathrm{~min}$, by 3-(2-benzothiazolyl)sulfanyl-2,2dimethylpropanal 8 ( $1.7 \mathrm{~g}, 6.8 \mathrm{mmol})$ in THF $(5 \mathrm{~mL})$. The mixture was stirred under reflux for $16 \mathrm{~h}$ then cooled to $\mathrm{rt}$ and diluted with EtOAc $(20 \mathrm{~mL})$. The black suspension was filtered through a plug of silica that was washed with ether $(4 \times 40 \mathrm{~mL})$. The filtrate was concentrated under reduced pressure and chromatography of the residue (80:20 light petroleum:ether) gave the title compound $12(2.11 \mathrm{~g}, 83 \%)$ as a yellow oil, $R_{\mathrm{f}}=$ 0.55 (70:30 light petroleum:EtOAc) (Found: $\mathrm{M}^{+}+\mathrm{H}, 376.0843$. $\mathrm{C}_{16} \mathrm{H}_{20} \mathrm{NO}_{3} \mathrm{~F}_{2} \mathrm{~S}_{2}$ requires $\left.\mathrm{M}, 376.0848\right) ; v_{\text {max }} / \mathrm{cm}^{-1} 3182,2956$, $1775,1459,1428,1307,1074,1004$ and 756; $\delta_{\mathrm{H}}(400 \mathrm{MHz}$, $\left.\mathrm{CDCl}_{3}\right) 0.97\left(3 \mathrm{H}, \mathrm{t}, J\right.$ 8.8, $\left.\mathrm{OCH}_{2} \mathrm{CH}_{3}\right), 1.15\left(3 \mathrm{H}, \mathrm{d}, J \mathrm{~J} .2,4-\mathrm{CH}_{3}\right)$, $1.23\left(3 \mathrm{H}, \mathrm{d}, J \mathrm{~J} 2.8,4-\mathrm{CH}_{3}\right), 2.69$ and 3.91 (each $1 \mathrm{H}, \mathrm{d}, J$ 14.4, 5$\mathrm{H}), 4.03(1 \mathrm{H}, \mathrm{t}, J 6.0,3-\mathrm{H}), 4.20-4.06\left(2 \mathrm{H}, \mathrm{m}, \mathrm{OCH}_{2}\right), 6.66(1 \mathrm{H}$, br. d, J 8.0, OH), 7.25 and 7.33 (each $1 \mathrm{H}, \mathrm{td}, J$ 7.5, 1.1, ArH) and 7.68-7.63 $(2 \mathrm{H}, \mathrm{m}, \mathrm{ArH}) ; \delta_{\mathrm{C}}\left(100 \mathrm{MHz}, \mathrm{CDCl}_{3}\right)$ 13.7, 18.8, 19.5, $36.1,46.4,62.5,71.4\left(\mathrm{t}^{2}{ }^{2} \mathrm{~J}_{\mathrm{C}-\mathrm{F}} 22\right), 117.5\left(\mathrm{t},{ }^{1} J_{\mathrm{C}-\mathrm{F}} 251\right), 120.5,121.2$, $124.9,126.5,134.7,151.6,164.1\left(\mathrm{t},{ }^{2} J_{\mathrm{C}-\mathrm{F}} 30\right)$ and $170.4 ; \delta_{\mathrm{F}}(376$ $\left.\mathrm{MHz}, \mathrm{CDCl}_{3}\right)-122.42$ and -103.68 (each d, $\left.{ }^{2} J_{\mathrm{F}-\mathrm{F}} 246.7\right) ; \mathrm{m} / \mathrm{z}\left(\mathrm{ES}^{+}\right)$ $398\left(\mathrm{M}^{+}+23,52 \%\right)$ and $376\left(\mathrm{M}^{+}+1,100\right)$.

Ethyl 5-benzyloxymethoxy-2,2-difluoro-3-hydroxy-4,4dimethylpentanoate (13). Following the procedure outlined for the synthesis of the hydroxyester 12, zinc powder $(60 \mathrm{mg}, 0.9$ $\mathrm{mmol}$ ), copper(I) chloride, ethyl bromodifluoroacetate 11 (0.11 
$\mathrm{mL}, 0.59 \mathrm{mmol}$ ) and 3-benzyloxymethoxy-2,2-dimethylpropanal $9(0.1 \mathrm{~g}, 0.45 \mathrm{mmol})$, after heating under reflux for $36 \mathrm{~h}$ and chromatography (85:15 light petroleum:EtOAc), gave the title compound $13(0.12 \mathrm{~g}, 75 \%)$ as a colourless oil, $R_{\mathrm{f}}=0.6(70: 30$ light petroleum:EtOAc) (Found: $\mathrm{M}^{+}+\mathrm{Na}, 369.1483$. $\mathrm{C}_{17} \mathrm{H}_{24} \mathrm{O}_{5} \mathrm{~F}_{2} \mathrm{Na}$ requires $\left.\mathrm{M}, 369.1485\right) ; v_{\text {max }} / \mathrm{cm}^{-1} 3458,2942$, 2884, 1759, 1455, 1308, 1045, 910 and 740; $\delta_{\mathrm{H}}\left(500 \mathrm{MHz}, \mathrm{CDCl}_{3}\right)$ 1.09 and 1.22 (each $\left.3 \mathrm{H}, \mathrm{s}, 4-\mathrm{CH}_{3}\right), 1.37\left(3 \mathrm{H}, \mathrm{t}, J \mathrm{~J} .5, \mathrm{OCH}_{2} \mathrm{CH}_{3}\right)$, 3.40 and 3.83 (each $1 \mathrm{H}, \mathrm{d}, J$ 7.5, 5-H), 3.93-4.02 (2 H, m, 3-H, $\mathrm{OH}$ ), $4.36\left(2 \mathrm{H}, \mathrm{q}, J 6.2, \mathrm{OCH}_{2}\right.$ ), 4.61 and 4.62 (each $1 \mathrm{H}, \mathrm{d}, J$ 12.5, $\mathrm{PhHCH}$ ), 4.76 and 4.77 (each $1 \mathrm{H}, \mathrm{d}, J$ 7.5, OHCHO) and 7.33-7.39 $(5 \mathrm{H}, \mathrm{m}, \mathrm{ArH}) ; \delta_{\mathrm{C}}\left(125 \mathrm{MHz}, \mathrm{CDCl}_{3}\right)$ 14.0, 18.2, 21.0, 24.2, 62.9, $69.9,76.5,76.8,94.8,117.0\left(t,{ }^{1} J_{C-F} 261\right), 127.9,128.5,137.3$ and $164.0\left(\mathrm{t},{ }^{2} J_{\mathrm{C}-\mathrm{F}} 30\right) ; \delta_{\mathrm{F}}\left(376 \mathrm{MHz}, \mathrm{CDCl}_{3}\right)-124.57$ and -105.93 (each $\left.d,{ }^{2} J_{F-F} 255.7\right) ; m / z\left(E S^{+}\right) 369\left(M^{+}+23,100 \%\right)$.

Ethyl 5-tert-butyldiphenylsilyloxy-2,2-difluoro-3-hydroxy4,4-dimethylpentanoate (14). The procedure outlined for the synthesis of the hydroxyester 12 using zinc powder $(0.61 \mathrm{~g}, 9.4$ $\mathrm{mmol})$, copper(I) chloride, ethyl bromodifluoroacetate 11 (0.8 $\mathrm{mL}, \quad 6.1 \mathrm{mmol}$ ) and 3-tert-butyldiphenylsilyloxy-2,2dimethylpropanal 10 (1.6 g, $4.7 \mathrm{mmol})$, after heating under reflux for $36 \mathrm{~h}$, an aqueous extraction using ether $(15 \mathrm{~mL})$, water $(10 \mathrm{~mL})$ and aqueous hydrogen chloride $(1 \mathrm{M}, 10 \mathrm{~mL})$, and with extraction of the aqueous layer using ether $(3 \times 15 \mathrm{~mL})$, gave, after chromatography (90:10 light petroleum:EtOAc), the title compound $14(0.7 \mathrm{~g}, 78 \%)$ as a colourless oil, $R_{\mathrm{f}}=0.7$ (70:30 light petroleum:EtOAc) (Found: $\mathrm{M}^{+}+\mathrm{Na}, 487.2089 . \mathrm{C}_{25} \mathrm{H}_{34} \mathrm{O}_{4} \mathrm{~F}_{2} \mathrm{SiNa}$ requires $M, 487.2087) ; v_{\max } / \mathrm{cm}^{-1} 3445,2931,2858,1761,1724$, $1428,1305,1110,1071,820$ and $739 ; \delta_{\mathrm{H}}\left(400 \mathrm{MHz}, \mathrm{CDCl}_{3}\right) 1.10$ [15 H, s, $\left.2 \times 4-\mathrm{CH}_{3}, \mathrm{SiC}\left(\mathrm{CH}_{3}\right)_{3}\right], 1.39\left(3 \mathrm{H}, \mathrm{t}, J\right.$ J $\left..0, \mathrm{OCH}_{2} \mathrm{CH}_{3}\right), 3.40$ and 3.89 (each $1 \mathrm{H}, \mathrm{d}, \mathrm{J}$ 12.0, 5-H), $4.10(1 \mathrm{H}, \mathrm{d}, \mathrm{J} 24.0,3-\mathrm{H}), 4.39$ $\left(2 \mathrm{H}, \mathrm{q}, J\right.$ 8.0, $\left.\mathrm{OCH}_{2}\right), 7.38-7.44(6 \mathrm{H}, \mathrm{m}, \mathrm{ArH})$ and 7.70-7.76 $(4 \mathrm{H}$, $\mathrm{m}, \mathrm{ArH}) ; \delta_{\mathrm{C}}\left(100 \mathrm{MHz}, \mathrm{CDCl}_{3}\right) 13.9,19.1,23.8,26.5,26.8,29.7$, $38.0,62.8,73.4,117.0\left(\mathrm{t},{ }^{1} J_{\mathrm{C}-\mathrm{F}} 258.0\right), 127.7,127.8,129.6,130.0$, 132.1, 134.8, 135.6, 135.7 and $164.1\left(\mathrm{t}^{2}{ }^{2} \mathrm{~J}_{\mathrm{C}-\mathrm{F}} 31.5\right) ; \mathrm{m} / \mathrm{z}\left(\mathrm{ES}^{+}\right) 487$ $\left(\mathrm{M}^{+}+23,100 \%\right)$.

Ethyl 5-(2-benzothiazolyl)sulfanyl-2,2-difluoro-4,4dimethyl-3-triethylsilyloxypentanoate (15). 2,6-Lutidine (1.3 $\mathrm{mL}, 11 \mathrm{mmol})$ and triethylsilyl triflate $(1.9 \mathrm{~mL}, 8.4 \mathrm{mmol})$ were added to the hydroxyester $12(2.1 \mathrm{~g}, 5.6 \mathrm{mmol})$ in DCM $(20 \mathrm{~mL})$ at $0{ }^{\circ} \mathrm{C}$ and the mixture stirred at $\mathrm{rt}$ for $24 \mathrm{~h}$. Water $(20 \mathrm{~mL})$ was added and the aqueous layer was extracted with DCM $(3 \times 20$ $\mathrm{mL})$. The organic extracts were dried $\left(\mathrm{MgSO}_{4}\right)$ and concentrated under reduced pressure. Chromatography of the residue $(80: 20$ light petroleum:EtOAc) gave the title compound 15 (2.05 g, 75\%) as a yellow oil, $R_{\mathrm{f}}=0.65$ (70:30 light petroleum:EtOAc) (Found: $\mathrm{M}^{+}+\mathrm{H}$, 490.1717. $\mathrm{C}_{22} \mathrm{H}_{34} \mathrm{NO}_{3} \mathrm{~F}_{2} \mathrm{~S}_{2} \mathrm{Si}$ requires $\mathrm{M}, 490.1712$ ); $v_{\text {max }} / \mathrm{cm}^{-1} 2955,1758,1459,1427,1307,1082,994,830$ and 726; $\delta_{\mathrm{H}}\left(400 \mathrm{MHz}, \mathrm{CDCl}_{3}\right)$ 0.61-0.66 $\left(6 \mathrm{H}, \mathrm{m}, 3 \times \mathrm{SiCH}_{2}\right), 0.93(9 \mathrm{H}, \mathrm{t}, \mathrm{J}$ 7.6, $3 \times \mathrm{CH}_{2} \mathrm{CH}_{3}$ ), 1.05 and 1.08 (each $\left.3 \mathrm{H}, \mathrm{s}, 4-\mathrm{CH}_{3}\right), 1.30(3 \mathrm{H}, \mathrm{t}, J$ 6.8, $\mathrm{OCH}_{2} \mathrm{CH}_{3}$ ), 3.38 and 3.47 (each $\left.1 \mathrm{H}, \mathrm{d}, J 10.0,5-\mathrm{H}\right), 4.13(1 \mathrm{H}$, $\mathrm{m}, 3-\mathrm{H}), 4.26\left(2 \mathrm{H}, \mathrm{q}, J\right.$ 6.8, $\left.\mathrm{OCH}_{2} \mathrm{CH}_{3}\right), 7.22(1 \mathrm{H}, \mathrm{t}, J$ 8.4, $\mathrm{ArH})$, $7.34(1 \mathrm{H}, \mathrm{t}, J \mathrm{~J} .6, \mathrm{ArH}), 7.67(1 \mathrm{H}, \mathrm{d}, J$ 8.0, $\mathrm{ArH})$ and $7.77(1 \mathrm{H}, \mathrm{d}$, J 8.4, $\mathrm{ArH}) ; \delta_{\mathrm{C}}\left(125 \mathrm{MHz}, \mathrm{CDCl}_{3}\right) 5.4,6.9,13.9,22.7,23.3,39.5$, $42.8,63.1,76.8,115.2\left(\mathrm{t},{ }^{1} J_{\mathrm{C}-\mathrm{F}} 255.0\right), 121.0,121.5,124.2,125.9$,
135.2, 153.2, $164.2\left(\mathrm{t}^{2} \mathrm{~J}_{\mathrm{C}-\mathrm{F}} 33.2\right)$ and $167.3 ; \delta_{\mathrm{F}}\left(376 \mathrm{MHz}, \mathrm{CDCl}_{3}\right)$ -111.42 and -105.89 (each d, $\left.{ }^{2} J_{\mathrm{F}-\mathrm{F}} 263.2\right) ; \mathrm{m} / \mathrm{z}\left(\mathrm{ES}^{+}\right) 512\left(\mathrm{M}^{+}+\right.$ $23,100 \%)$ and $490\left(\mathrm{M}^{+}+1,61\right)$.

Ethyl 5-benzyloxymethoxy-2,2-difluoro-4,4-dimethyl-3triethylsilyloxypentanoate (16). Following the procedure outlined for the preparation of the silyl ether 15, the hydroxyester $13(0.2 \mathrm{~g}, 0.58 \mathrm{mmol}), 2,6$-lutidine $(0.2 \mathrm{~mL}, 1.7$ $\mathrm{mmol}$ ) and triethylsilyl triflate $(0.2 \mathrm{~mL}, 0.87 \mathrm{mmol})$ with stirring for $10 \mathrm{~d}$, after chromatography (85:15 light petroleum:EtOAc) gave the title compound $16(0.24 \mathrm{~g}, 85 \%)$ as a colourless oil, $R_{\mathrm{f}}=$ 0.75 (70:30 light petroleum:EtOAc) (Found: $\mathrm{M}^{+}+\mathrm{Na}, 483.2337$. $\mathrm{C}_{23} \mathrm{H}_{38} \mathrm{O}_{5} \mathrm{~F}_{2} \mathrm{SiNa}$ requires $\left.\mathrm{M}, 483.2349\right) ; v_{\max } / \mathrm{cm}^{-1} 2972,2878$, $1760,1455,1379,1306,1086,1046,879,834$ and $732 ; \delta_{H}(500$ $\left.\mathrm{MHz}, \mathrm{CDCl}_{3}\right)$ 0.65-0.69 $\left(6 \mathrm{H}, \mathrm{m}, 3 \times \mathrm{SiCH}_{2}\right), 0.92-1.03[15 \mathrm{H}, \mathrm{m}, 2 \times$ $\left.4-\mathrm{CH}_{3}, 3 \times \mathrm{SiCH}_{2} \mathrm{CH}_{3}\right), 1.37\left(3 \mathrm{H}, \mathrm{t}, J 7.5, \mathrm{OCH}_{2} \mathrm{CH}_{3}\right), 3.31$ and 3.44 (each $1 \mathrm{H}, \mathrm{d}, \mathrm{J}$ 10.0, 5-H), 4.24-4.34 $\left(3 \mathrm{H}, \mathrm{m}, 3-\mathrm{H}, \mathrm{OCH}_{2}\right), 4.58$ and 4.62 (each $1 \mathrm{H}, \mathrm{d}, J$ 10.0, $\mathrm{PhHCH}$ ), 4.73 and 4.75 (each $1 \mathrm{H}, \mathrm{d}, J$ 5.0, $\mathrm{OHCHO})$ and 7.32-7.37 $(5 \mathrm{H}, \mathrm{m}, \mathrm{ArH}) ; \delta_{\mathrm{C}}\left(100 \mathrm{MHz}, \mathrm{CDCl}_{3}\right)$ 5.1, 6.8, 13.8, 18.0, 20.5, 21.2, 38.9, 62.7, 69.3, 74.7, 75.1, 94.7, $115.7\left(\mathrm{t},{ }^{1} J_{\mathrm{C}-\mathrm{F}} 261\right), 127.7,127.8,128.4,137.8$ and $164.4\left(\mathrm{t},{ }^{2} J_{\mathrm{C}-\mathrm{F}}\right.$ 30); $\delta_{F}(376 \mathrm{MHz})-111.35$ and -106.22 (each d, $\left.{ }^{1} J_{F-F} 263.2\right) ; \mathrm{m} / z$ $\left(\mathrm{ES}^{+}\right) 483\left(\mathrm{M}^{+}+23,75 \%\right)$ and $369(100)$.

Ethyl 5-tert-butyldiphenylsilyloxy-2,2-difluoro-4,4dimethyl-3-triethylsilyloxypentanoate (17). Following the procedure outlined for the preparation of the silyl ether 15 , the hydroxyester $14(0.2 \mathrm{~g}, 0.43 \mathrm{mmol}), 2,6$-lutidine $(0.12 \mathrm{~mL}, 1.0$ $\mathrm{mmol}$ ) and triethylsilyl triflate $(0.2 \mathrm{~mL}, 0.86 \mathrm{mmol})$ with stirring for 10 days, after chromatography (85:15 light petroleum:EtOAc) gave the title compound $\mathbf{1 7}(0.23 \mathrm{~g}, 94 \%)$ as a colourless oil, $R_{\mathrm{f}}=0.8$ (70:30 light petroleum:EtOAc) (Found: $\mathrm{M}^{+}$ $+\mathrm{Na}, 601.2957 . \mathrm{C}_{31} \mathrm{H}_{48} \mathrm{O}_{4} \mathrm{~F}_{2} \mathrm{Si}_{2} \mathrm{Na}$ requires $\left.\mathrm{M}, 601.2952\right) ; v_{\max } / \mathrm{cm}^{-}$ ${ }^{1}$ 2886, 1670, 1432, 1243 and 1032; $\delta_{\mathrm{H}}\left(400 \mathrm{MHz}, \mathrm{CDCl}_{3}\right)$ 0.50$0.62\left(6 \mathrm{H}, \mathrm{m}, 3 \times \mathrm{SiCH}_{2}\right), 0.88-0.97\left(9 \mathrm{H}, \mathrm{m}, 3 \times \mathrm{SiCH}_{2} \mathrm{CH}_{3}\right), 1.07-$ $1.10\left[15 \mathrm{H}, \mathrm{s}, 2 \times 4-\mathrm{CH}_{3}, \mathrm{SiC}\left(\mathrm{CH}_{3}\right)_{3}\right], 1.34\left(3 \mathrm{H}, \mathrm{t}, J 7.0, \mathrm{OCH}_{2} \mathrm{CH}_{3}\right)$, 3.30 and 3.50 (each $1 \mathrm{H}, \mathrm{d}, J$ 12.0, 5-H), 4.27-4.39 (3 H, m, 3-H, $\left.\mathrm{OCH}_{2}\right), 7.38-7.45(6 \mathrm{H}, \mathrm{m}, \mathrm{ArH})$ and 7.64-7.67 (4 H, m, ArH); $m / z$ $\left(\mathrm{ES}^{+}\right) 601\left(\mathrm{M}^{+}+23,10 \%\right), 565(50)$ and $487(100)$.

6-(2-Benzothiazolyl)sulfanyl-3,3-difluoro-5,5-dimethyl-4triethylsilyloxyhexan-2-one (18). Methyllithium (1.0 M, $4.0 \mathrm{~mL}$, $4.0 \mathrm{mmol})$ was added to the ester $15(1.3 \mathrm{~g}, 2.66 \mathrm{mmol})$ in THF (30 mL) at $-78{ }^{\circ} \mathrm{C}$ and the solution was stirred at $-78{ }^{\circ} \mathrm{C}$ for 5.5 h. Water $(20 \mathrm{~mL})$ was added followed by ether $(20 \mathrm{~mL})$ and the aqueous layer was extracted with ether $(3 \times 10 \mathrm{~mL})$. The organic extracts were dried $\left(\mathrm{MgSO}_{4}\right)$ and concentrated under reduced pressure. Chromatography of the residue (90:10 light petroleum:EtOAc) gave the title compound $\mathbf{1 8}(0.93 \mathrm{~g}, 77 \%)$ as a yellow oil, $R_{\mathrm{f}}=0.7$ (70:30 light petroleum:EtOAC) (Found: $\mathrm{M}^{+}+$ $\mathrm{H}, 460.1617$. $\mathrm{C}_{21} \mathrm{H}_{32} \mathrm{NO}_{2} \mathrm{~F}_{2} \mathrm{~S}_{2} \mathrm{Si}$ requires $\left.\mathrm{M}, 460.1607\right) ; \delta_{\mathrm{H}}(400$ $\left.\mathrm{MHz}, \mathrm{CDCl}_{3}\right)$ 0.61-0.67 $\left(6 \mathrm{H}, \mathrm{m}, 3 \times \mathrm{SiCH}_{2}\right), 0.93(9 \mathrm{H}, \mathrm{t}, J$ 8.4, $3 \times$ $\mathrm{SiCH}_{2} \mathrm{CH}_{3}$ ), 1.03 and 1.05 (each $\left.3 \mathrm{H}, \mathrm{s}, 5-\mathrm{CH}_{3}\right), 2.32(3 \mathrm{H}, \mathrm{t}, \mathrm{J} 1.8$, 1- $\mathrm{H}_{3}$ ), 3.49 and 3.53 (each $1 \mathrm{H}, \mathrm{d}, J$ 12.0, 6-H), $4.14(1 \mathrm{H}, \mathrm{t}, J 12.8$, 4-H), $7.21(1 \mathrm{H}, \mathrm{td}, J$ 8.4, 1.2, $\mathrm{ArH}), 7.33(1 \mathrm{H}, \mathrm{td}, J \mathrm{7.8}, 1.2, \mathrm{ArH})$, $7.67(1 \mathrm{H}, \mathrm{d}, J$ 7.8, ArH $)$ and $7.77(1 \mathrm{H}, \mathrm{d}, J$ 8.0, $\mathrm{ArH}) ; \delta_{\mathrm{C}}(100$ $\mathrm{MHz}_{2} \mathrm{CDCl}_{3}$ ) 5.1, 6.9, 23.1, 23.5, 26.2, 31.0, 39.4, 42.7, 76.6, 
116.9, 121.0, 121.4, 124.2 126.0, 135.3, 153.1, 167.3 and 199.6 (d, $\left.{ }^{2} J_{\mathrm{C}-\mathrm{F}} 30\right) ; \delta_{\mathrm{F}}\left(376 \mathrm{MHz}, \mathrm{CDCl}_{3}\right.$ ) -110.73 and -107.56 (each d, $\left.{ }^{2} J_{F-F} 270.7\right) ; m / z\left(E^{+}\right) 482\left(\mathrm{M}^{+}+23,100 \%\right)$ and $460\left(\mathrm{M}^{+}+1,55\right)$.

Methyllithium $(1.0 \mathrm{M}, 0.07 \mathrm{~mL}, 0.7 \mathrm{mmol})$ was added to the ester 15 (0.13 g, $0.27 \mathrm{mmol})$ in THF $(2 \mathrm{~mL})$ at $0{ }^{\circ} \mathrm{C}$ and the solution was stirred at $\mathrm{rt}$ for $1.5 \mathrm{~h}$. Water $(10 \mathrm{~mL})$ was added followed by ether $(10 \mathrm{~mL})$ and the aqueous layer was extracted with ether $(3 \times 10 \mathrm{~mL})$. The organic extracts were dried $\left(\mathrm{MgSO}_{4}\right)$ and concentrated under reduced pressure. Chromatography of the residue (80:20 light petroleum:ether) gave 6-(2benzothiazolyl)sulfanyl-3,3-difluoro-4-triethylsilyloxy-2,5,5-

trimethylhexan-2-ol ( $86 \mathrm{mg}, 72 \%$ ) as a colourless oil, $R_{\mathrm{f}}=0.5$ (70:30 light petroleum:EtOAc) (Found: $\mathrm{M}^{+}+\mathrm{H}, 476.1922$. $\mathrm{C}_{22} \mathrm{H}_{36} \mathrm{NO}_{2} \mathrm{~F}_{2} \mathrm{~S}_{2} \mathrm{Si}$ requires $\left.\mathrm{M}, 476.1920\right) ; v_{\max } / \mathrm{cm}^{-1} 3452,2954$, $1536,1458,1428,1238,1159,1070,1003$ and 740; $\delta_{H}(500$ $\left.\mathrm{MHz}, \mathrm{CDCl}_{3}\right)$ 0.44-0.46 (6 H, m, $\left.3 \times \mathrm{SiCH}_{2}\right), 0.74(9 \mathrm{H}, \mathrm{t}, \mathrm{J}$ 10.0, $3 \times$ $\mathrm{CH}_{2} \mathrm{CH}_{3}$ ), 1.14 and 1.19 (each $3 \mathrm{H}, \mathrm{s}, 5-\mathrm{CH}_{3}$ ), 1.28 and 1.31 (each $3 \mathrm{H}, \mathrm{s}, 1-\mathrm{H}_{3}$ or $\left.2-\mathrm{CH}_{3}\right), 3.01$ and 3.86 (each $1 \mathrm{H}, \mathrm{d}, J 15.0,6-\mathrm{H}$ ), $4.15(1 \mathrm{H}, \mathrm{dd}, J 20.0,5.0,4-\mathrm{H}), 5.56(1 \mathrm{H}, \mathrm{d}, J 5.0, \mathrm{OH}), 7.22$ and 7.34 (each $1 \mathrm{H}, \mathrm{t}, J$ 10.0, ArH) and 7.66 and 7.73 (each $1 \mathrm{H}, \mathrm{d}, J$ 10.0, $\mathrm{ArH}$ ); $\delta_{\mathrm{F}}\left(376 \mathrm{MHz}, \mathrm{CDCl}_{3}\right)-124.40$ and -113.61 (each d, ${ }^{2} \mathrm{~J}_{\mathrm{F}-}$ F 225.6); $m / z\left(\mathrm{ES}^{+}\right) 476\left(\mathrm{M}^{+}+1,100 \%\right)$.

\section{6-Benzyloxymethyloxy-3,3-difluoro-5,5-dimethyl-4-}

triethylsilyloxyhexan-2-one (19). Following the procedure outlined for the synthesis of the ketone 18, the ester 16 (50 mg, $0.11 \mathrm{mmol})$ in THF $(2 \mathrm{~mL})$ and methyllithium $(1.0 \mathrm{M}, 0.16 \mathrm{~mL}$, $0.16 \mathrm{mmol})$, after chromatography (90:10 light petroleum: EtOAc) gave the title compound 19 (44 mg, 86\%) as a yellow oil, $R_{\mathrm{f}}=0.7$ (70:30 light petroleum:EtOAc) (Found: $\mathrm{M}^{+}+\mathrm{Na}$, 453.2245. $\mathrm{C}_{22} \mathrm{H}_{36} \mathrm{O}_{4} \mathrm{~F}_{2} \mathrm{SiNa}$ requires $\left.\mathrm{M}, 453.2244\right) ; v_{\max } / \mathrm{cm}^{-1} 2993$, 2864, 1783, 1432, 1304, 1048, 1032 and 728; $\delta_{\mathrm{H}}(400 \mathrm{MHz}$, $\left.\mathrm{CDCl}_{3}\right)$ 0.53-0.82 (6 H, m, $\left.3 \times \mathrm{SiCH}_{2}\right), 0.93-1.03(15 \mathrm{H}, \mathrm{m}, 2 \times 5-$ $\left.\mathrm{CH}_{3}, 3 \times \mathrm{CH}_{2} \mathrm{CH}_{3}\right), 2.36\left(3 \mathrm{H}, \mathrm{s}, 1-\mathrm{H}_{3}\right), 3.34$ and 3.37 (each $1 \mathrm{H}, \mathrm{d}, J$ 8.0, 6-H), $4.26(1 \mathrm{H}, \mathrm{m}, 4-\mathrm{H}$ ), 4.56 and 4.62 (each $1 \mathrm{H}, \mathrm{d} J$ 12.0, $\mathrm{PhHCH}$ ), 4.70 and 4.72 (each $1 \mathrm{H}, \mathrm{d} J 8.0, \mathrm{OHCHO}$ ) and 7.27-7.38 $(5 \mathrm{H}, \mathrm{m}, \mathrm{ArH}) ; \delta_{\mathrm{C}}\left(100 \mathrm{MHz}, \mathrm{CDCl}_{3}\right)$ 5.0, 6.8, 17.9, 20.9, 21.6, 26.0, $38.8,69.4,74.2,74.8\left(\mathrm{t},{ }^{2} J_{C-F} 24\right), 94.5,117.1\left(\mathrm{t},{ }^{1} J_{C-F} 257.0\right)$, 127.7, 127.8, 128.4, 137.7 and $199.4\left(t,{ }^{2} J_{C-F} 30.5\right) ; \delta_{F}(376 \mathrm{MHz}$, $\left.\mathrm{CDCl}_{3}\right)-109.85$ and -108.48 (each d, $\left.{ }^{2} J_{\mathrm{F}-\mathrm{F}}, 270.7\right) ; \mathrm{m} / \mathrm{z}\left(\mathrm{ES}^{+}\right) 453$ $\left(\mathrm{M}^{+}+23,100 \%\right)$.

\section{6-tert-Butyldiphenylsilyloxy-3,3-difluoro-5,5-dimethyl-4-} triethylsilyloxyhexan-2-one (20). Following the procedure outlined for the synthesis of the ketone 18 , the ester $17(0.5 \mathrm{~g}$, $0.8 \mathrm{mmol})$ in THF $(20 \mathrm{~mL})$ and methyllithium $(1.0 \mathrm{M}, 1.3 \mathrm{~mL}, 1.3$ $\mathrm{mmol})$, after chromatography (90:10 light petroleum: EtOAc) gave the title compound $\mathbf{2 0}(0.22 \mathrm{~g}, 55 \%)$ as a colourless oil, $R_{\mathrm{f}}=$ 0.8 (70:30 light petroleum:EtOAc) (Found: $\mathrm{M}^{+}+\mathrm{Na}, 571.2854$. $\mathrm{C}_{30} \mathrm{H}_{46} \mathrm{O}_{3} \mathrm{~F}_{2} \mathrm{Si}_{2} \mathrm{Na}$ requires $\left.\mathrm{M}, 571.2846\right) ; v_{\max } / \mathrm{cm}^{-1} 2980,1724$, 1423, 1201 and 1109; $\delta_{\mathrm{H}}\left(500 \mathrm{MHz}, \mathrm{CDCl}_{3}\right)$ 0.55-0.65 $(6 \mathrm{H}, \mathrm{m}, 3 \times$ $\left.\mathrm{SiCH}_{2}\right), 0.92\left(9 \mathrm{H}, \mathrm{t}, J\right.$ J 6.0, $\left.3 \times \mathrm{SiCH}_{2} \mathrm{CH}_{3}\right), 1.09\left(3 \mathrm{H}, \mathrm{s}, 5-\mathrm{CH}_{3}\right), 1.12$ [9 H, s, SiC $\left.\left(\mathrm{CH}_{3}\right)_{3}\right], 1.28\left(3 \mathrm{H}, \mathrm{s}, 5-\mathrm{CH}_{3}\right), 2.34\left(3 \mathrm{H}, \mathrm{s}, 1-\mathrm{H}_{3}\right), 3.35$ and 3.48 (each $1 \mathrm{H}, \mathrm{d}, J \mathrm{~J}$ 10.0, 6-H), $4.35(1 \mathrm{H}, \mathrm{t}, J$ 10.0, 4-H), 7.37$7.44(6 \mathrm{H}, \mathrm{m}, \mathrm{ArH})$ and 7.66-7.68 $(4 \mathrm{H}, \mathrm{m}, \mathrm{ArH}) ; \delta_{\mathrm{c}}(100 \mathrm{MHz}$, $\mathrm{CDCl}_{3}$ ) 6.5, 6.9, 19.2, 19.4, 20.9, 26.2, 26.9, 40.1, 70.0, 74.3 (t, $\left.{ }^{2} J_{C-F} 32\right), 127.4,127.6,134.3,134.4,135.1,135.5,135.8,135.9$ and $199.8\left(\mathrm{t},{ }^{2} \mathrm{~J}_{\mathrm{C}-\mathrm{F}} 30\right) ; \mathrm{m} / \mathrm{z}\left(\mathrm{ES}^{+}\right) 571\left(\mathrm{M}^{+}+23,100 \%\right)$.

6-Benzyloxymethoxy-3,3-difluoro-4-triethylsilyloxy-2,5,5trimethylhex-1-ene (21). Potassium hexamethyldisilazide $(1.0 \mathrm{M}$ in THF, $0.5 \mathrm{~mL}, 0.5 \mathrm{mmol}$ ) was added to methyl(triphenyl)phosphonium bromide $(0.2 \mathrm{~g}, 0.56 \mathrm{mmol})$ in toluene $(2 \mathrm{~mL})$ at $0{ }^{\circ} \mathrm{C}$ and the solution was stirred for $30 \mathrm{~min}$. The ketone 19 ( $80 \mathrm{mg}, 0.19 \mathrm{mmol})$ in toluene $(1 \mathrm{~mL})$ was added and the mixture was stirred at rt for $48 \mathrm{~h}$. Water $(2 \mathrm{~mL})$ was added followed by EtOAc $(5 \mathrm{~mL})$ and the aqueous phase was extracted with EtOAc $(3 \times 5 \mathrm{~mL})$. The organic extracts were dried $\left(\mathrm{MgSO}_{4}\right)$ and concentrated under reduced pressure. Chromatography of the residue (90:10 light petroleum:ether) gave the title compound $\mathbf{2 1}(60 \mathrm{mg}, 75 \%)$ as a colourless oil, $R_{\mathrm{f}}=$ 0.9 (70:30 light petroleum:EtOAc) (Found: $\mathrm{M}^{+}+\mathrm{Na}, 451.2458$. $\mathrm{C}_{23} \mathrm{H}_{38} \mathrm{O}_{3} \mathrm{~F}_{2} \mathrm{SiNa}$ requires $\left.\mathrm{M}, 451.2451\right) ; v_{\text {max }} / \mathrm{cm}^{-1} 2954,2877$, $1455,1379,1239,1159,1105,1047,1025,1002,921,833$ and 729; $\delta_{\mathrm{H}}\left(500 \mathrm{MHz}, \mathrm{CDCl}_{3}\right)$ 0.65-0.71 $\left(6 \mathrm{H}, \mathrm{m}, 3 \times \mathrm{SiCH}_{2}\right), 0.96-1.03$ $\left(15 \mathrm{H}, \mathrm{m}, 2 \times 5-\mathrm{CH}_{3}, 3 \times \mathrm{CH}_{2} \mathrm{CH}_{3}\right), 1.91\left(3 \mathrm{H}, \mathrm{s}, 2-\mathrm{CH}_{3}\right), 3.31$ and 3.41 (each $1 \mathrm{H}, \mathrm{d}, J$ 10.0, 6-H), $4.05(1 \mathrm{H}, \mathrm{t}, J$ 12.0, 4-H), 4.57 and 4.64 (each $1 \mathrm{H}, \mathrm{d}, J$ 10.0, PhHCH), 4.74 and 4.75 (each $\mathrm{H}, \mathrm{d}, J$ 7.0, $\mathrm{OHCHO}$ ), 5.15 and 5.40 (each $1 \mathrm{H}, \mathrm{s}, 1-\mathrm{H})$ and 7.28-7.37 $(5 \mathrm{H}, \mathrm{m}$, $\mathrm{ArH}) ; \delta_{\mathrm{C}}\left(125 \mathrm{MHz}, \mathrm{CDCl}_{3}\right)$ 5.0, 7.0, 19.1, 20.6, 21.1, 30.3, 38.8, $69.3,75.0,76.6\left(\mathrm{t}^{2} \mathrm{~J}_{\mathrm{C}-\mathrm{F}} 24.0\right), 94.7,116.2\left(\mathrm{t},{ }^{3} \mathrm{~J}_{\mathrm{C}-\mathrm{F}} 9.0\right), 121.3\left(\mathrm{t},{ }^{1} J_{\mathrm{C}-}\right.$ F 245.6), 127.7, 127.8, 128.4, 137.9 and 140.6 (t, $\left.{ }^{2} J_{C-F} 24.0\right) ; \delta_{F}$ (376 MHz, CDCl ${ }_{3}$ ) -98.90 and -98.69 (each d, $\left.{ }^{2} J_{F-F} 244\right) ; m / z\left(\mathrm{ES}^{+}\right)$ $451\left(\mathrm{M}^{+}+23,100 \%\right)$.

6-tert-Butyldiphenylsilyloxy-3,3-difluoro-4-triethylsilyloxy2,5,5-trimethylhex-1-ene (22). Following the procedure outlined for the synthesis of the alkene 21, methyl(triphenyl)phosphonium bromide $(0.16 \mathrm{~g}, 0.45 \mathrm{mmol})$ in toluene $(2 \mathrm{~mL})$, potassium hexamethyldisilazide $(1.0 \mathrm{M}$ in THF, $0.41 \mathrm{~mL}, 0.41 \mathrm{mmol})$ and the ketone $20(84 \mathrm{mg}, 0.15 \mathrm{mmol})$ in toluene $(1 \mathrm{~mL})$, after chromatography $(90: 10$ light petroleum:EtOAc) gave the title compound 22 (61 mg, 73\%) as a colourless oil, $R_{\mathrm{f}}=0.9$ (70:30 light petroleum:EtOAc) (Found: $\mathrm{M}^{+}$ $+\mathrm{Na}, 569.3050 . \mathrm{C}_{31} \mathrm{H}_{48} \mathrm{O}_{2} \mathrm{~F}_{2} \mathrm{Si}_{2} \mathrm{Na}$ requires $\left.\mathrm{M}, 569.3054\right) ; v_{\text {max }} / \mathrm{cm}^{-}$ ${ }^{1} 2955,1466,1107$ and 826; $\delta_{\mathrm{H}}\left(400 \mathrm{MHz}, \mathrm{CDCl}_{3}\right)$ 0.55-0.67 $(6 \mathrm{H}$, $\left.\mathrm{m}, 3 \times \mathrm{SiCH}_{2}\right), 0.92-0.98\left(9 \mathrm{H}, \mathrm{m}, 3 \times \mathrm{SiCH}_{2} \mathrm{CH}_{3}\right), 1.11(3 \mathrm{H}, \mathrm{s}, 5-$ $\left.\mathrm{CH}_{3}\right), 1.13\left[9 \mathrm{H}, \mathrm{s}, \mathrm{SiC}\left(\mathrm{CH}_{3}\right)_{3}\right], 1.30\left(3 \mathrm{H}, \mathrm{s}, 5-\mathrm{CH}_{3}\right), 1.89(3 \mathrm{H}, \mathrm{s}, 2-$ $\left.\mathrm{CH}_{3}\right), 3.40$ and 3.51 (each $1 \mathrm{H}, \mathrm{d}, J$ 8.0, 6- $\mathrm{H}$ ), $4.09(1 \mathrm{H}, \mathrm{t}, J$, 8.0, 4$\mathrm{H}), 5.08$ and 5.33 (each $1 \mathrm{H}, \mathrm{s}, 1-\mathrm{H}), 7.35-7.48(6 \mathrm{H}, \mathrm{m}, \mathrm{ArH}$ ) and 7.68-7.73 $(4 \mathrm{H}, \mathrm{m}, \mathrm{ArH}) ; \delta_{\mathrm{C}}\left(100 \mathrm{MHz}, \mathrm{CDCl}_{3}\right) 6.9,7.0,19.1,19.3$, $20.5,26.9,40.3,70.2,72.9,74.1,116.1,121.2\left(\mathrm{t},{ }^{2} J_{\mathrm{C}-\mathrm{F}} 23\right), 127.4$, 128.6, 129.6, 132.0, 135.7, 137.3 and $140.6 ; \delta_{\mathrm{F}}\left(376 \mathrm{MHz}_{2} \mathrm{CDCl}_{3}\right)$ -98.9 and $-98.2\left(\mathrm{t}^{2}{ }^{2} \mathrm{F-F} 248\right) ; \mathrm{m} / z\left(\mathrm{ES}^{+}\right) 569\left(\mathrm{M}^{+}+23,20 \%\right)$ and 293 (100).

2-Bromomethyl-6-tert-butyldiphenylsilyloxy-3,3-difluoro-4triethylsilyloxy-5,5-dimethylhex-1-ene (23). NBromosuccinimide $(12 \mathrm{mg}, 0.065 \mathrm{mmol}$ ) was added to the alkene 22 (30 mg, $0.055 \mathrm{mmol}$ ) in chloroform (1 mL). The suspension was exposed to UV light for $10 \mathrm{~min}$ and then heated under reflux for $24 \mathrm{~h}$. After cooling to rt, the mixture was concentrated under reduced pressure. Chromatography of the 
residue (90:10 light petroleum:EtOAc) gave the title compound 23 (25 mg, 72\%) as a colourless oil, $R_{\mathrm{f}}=0.9$ (70:30 light petroleum:EtOAc) (Found: $\mathrm{M}^{+}+\mathrm{Na}$, 647.2169. $\mathrm{C}_{31} \mathrm{H}_{47} \mathrm{O}_{2}{ }^{79} \mathrm{BrF}_{2} \mathrm{Si}_{2} \mathrm{Na}$ requires $\mathrm{M}, 647.2159$; Found: $\mathrm{M}^{+}+\mathrm{Na}$, 649.1790. $\mathrm{C}_{31} \mathrm{H}_{47} \mathrm{O}_{2}{ }^{81} \mathrm{BrF}_{2} \mathrm{Si}_{2} \mathrm{Na}$ requires $\left.\mathrm{M}, 649.2159\right) ; v_{\text {max }} / \mathrm{cm}^{-1}$ 2956, 2876, 1471, 1428, 1107, 1078, 1006, 822, 739 and 700; $\delta_{H}$ $\left(400 \mathrm{MHz}, \mathrm{CDCl}_{3}\right)$ 0.57-0.64 $\left(6 \mathrm{H}, \mathrm{m}, 3 \times \mathrm{SiCH}_{2}\right), 0.88-0.96(9 \mathrm{H}$, $\mathrm{m}, 3 \times \mathrm{SiCH}_{2} \mathrm{CH}_{3}$ ] $1.08\left[9 \mathrm{H}, \mathrm{s}, \mathrm{SiC}\left(\mathrm{CH}_{3}\right)_{3}\right], 1.11$ and 1.27 (each 3 $\left.\mathrm{H}, \mathrm{s}, 5-\mathrm{CH}_{3}\right), 3.32-3.49\left(2 \mathrm{H}, \mathrm{m}, 6-\mathrm{H}_{2}\right), 4.06-4.11(3 \mathrm{H}, \mathrm{m}, 4-\mathrm{H}, 2-$ $\left.\mathrm{CH}_{2}\right)$, 5.67-5.70 $\left(2 \mathrm{H}, \mathrm{m}, 1-\mathrm{H}_{2}\right), 7.38-7.45(6 \mathrm{H}, \mathrm{m}, \mathrm{ArH})$ and 7.64$7.72(4 \mathrm{H}, \mathrm{m}, \mathrm{ArH}) ; \delta_{\mathrm{F}}\left(376 \mathrm{MHz}, \mathrm{CDCl}_{3}\right)-98.96$ and -91.89 (each $\left.\mathrm{d}^{2}{ }^{2} \mathrm{~F}_{\mathrm{F}-\mathrm{F}} 256\right) ; \mathrm{m} / \mathrm{z}\left(\mathrm{ES}^{+}\right) 649\left(\mathrm{M}^{+}+23,20 \%\right), 647\left(\mathrm{M}^{+}+23,5\right)$ and $258(100)$.

\section{2-lodo-4-tert-butyldimethylsilyloxyhex-1-ene}

(29).

Trimethylsilyl chloride $(0.52 \mathrm{~mL}, 4.0 \mathrm{mmol})$ and water $(0.037 \mathrm{~mL}$, $2.0 \mathrm{mmol}$ ) were added to sodium iodide $(0.62 \mathrm{~g}, 4.0 \mathrm{mmol})$ in acetonitrile $(4 \mathrm{~mL})$ and the yellow suspension was stirred at $\mathrm{rt}$ for $20 \mathrm{~min}$. Hex-5-yn-3-ol $27(0.2 \mathrm{~mL}, 1.8 \mathrm{mmol}$ ) in acetonitrile (1 $\mathrm{mL}$ ) was added dropwise and the mixture stirred for $4 \mathrm{~h}$. Water $(5 \mathrm{~mL})$ was added and the mixture was extracted with ether $(3 \times$ $10 \mathrm{~mL})$. The organic extracts were dried $\left(\mathrm{MgSO}_{4}\right)$ and concentrated under reduced pressure. Chromatography of the residue (light petroleum to 90:10 light petroleum:EtOAc) gave 5iodohex-5-en-3-ol $28(0.33 \mathrm{~g}, 80 \%)$ as a colourless oil, $R_{\mathrm{f}}=0.55$ (70:30 light petroleum:EtOAc); $v_{\max } / \mathrm{cm}^{-1} 3402,2964,2933$, $1710,1617,1461,1205,1113,1021,979$ and $899 ; \delta_{H}(400 \mathrm{MHz}$, $\left.\mathrm{CDCl}_{3}\right) 1.00\left(3 \mathrm{H}, \mathrm{t}, J\right.$ 8.0, 1- $\left.\mathrm{H}_{3}\right), 1.56\left(2 \mathrm{H}, \mathrm{m}, 2-\mathrm{H}_{2}\right), 1.63(1 \mathrm{H}, \mathrm{br}$. s, OH), $2.46(1 \mathrm{H}, \mathrm{dd}, J 12.0,8.0,4-\mathrm{H}), 2.56(1 \mathrm{H}, \mathrm{dd}, J$ J 12.0, 3.0, 4- $\left.\mathrm{H}^{\prime}\right), 3.82(1 \mathrm{H}, \mathrm{m}, 3-\mathrm{H})$ and 5.86 and 6.18 (each $\left.1 \mathrm{H}, \mathrm{s}, 6-\mathrm{H}\right) ; \delta_{\mathrm{C}}$ $\left(100 \mathrm{MHz} \mathrm{CDCl}_{3}\right)$ 9.9, 28.9, 52.4, 71.3, 107.7 and 128.7 .

Imidazole $(0.21 \mathrm{~g}, 3.1 \mathrm{mmol})$ and tert-butyldimethylsilyl chloride $(0.26 \mathrm{~g}, 1.7 \mathrm{mmol})$ were added to 5-iodohex-5-en-3-ol $28(0.32 \mathrm{~g}, 1.4 \mathrm{mmol})$ in $\mathrm{DCM}(10 \mathrm{~mL})$ and the solution stirred at rt for $24 \mathrm{~h}$. Water $(10 \mathrm{~mL})$ was added, the aqueous layer was extracted with DCM $(3 \times 10 \mathrm{~mL})$ and the organic extracts were dried $\left(\mathrm{MgSO}_{4}\right)$ and concentrated under reduced pressure. Chromatography of the residue (90:10 light petroleum:EtOAc) gave the title compound $29(0.36 \mathrm{~g}, 75 \%)$ as a pale yellow oil, $R_{\mathrm{f}}$ $=0.6$ (70:30 light petroleum:EtOAc); $v_{\max } / \mathrm{cm}^{-1} 2956,2928,2856$, $1618,1462,1361,1252,1101,1033,894,835$ and $774 ; \delta_{H}(300$ $\mathrm{MHz}, \mathrm{CDCl}_{3}$ ) 0.09 and 0.10 (each $3 \mathrm{H}, \mathrm{s}, \mathrm{SiCH}_{3}$ ), 0.87-0.90 [12 H, $\left.\mathrm{m}, 6-\mathrm{H}_{3}, \mathrm{SiC}\left(\mathrm{CH}_{3}\right)_{3}\right], 1.44-1.56\left(2 \mathrm{H}, \mathrm{m}, 5-\mathrm{H}_{2}\right), 2.47-2.51(2 \mathrm{H}, \mathrm{m}, 3-$ $\left.\mathrm{H}_{2}\right), 3.86(1 \mathrm{H}, \mathrm{m}, 4-\mathrm{H})$ and 5.74 and 6.07 (each $\left.1 \mathrm{H}, \mathrm{s}, 1-\mathrm{H}\right) ; \delta_{\mathrm{C}}$ $\left(100 \mathrm{MHz} \mathrm{CDCl}_{3}\right)-4.6,-4.4,9.2,18.1,25.8,29.1,52.4,71.8$, 108.7 and 127.8 .

\section{Ethyl}

5-tert-butyldimethylsilyloxy-2,2-difluoro-3methyleneheptanoate (30). Copper powder (65 mg, $1.02 \mathrm{mmol}$ ) was added to a vigorously stirred solution of ethyl 2-iodo-2,2difluoroacetate $25(0.025 \mathrm{~mL}, 0.170 \mathrm{mmol})$ in anhydrous DMSO $(0.20 \mathrm{~mL})$. After $3 \mathrm{~h}$, the alkenyl iodide 29 (19 $\mathrm{mg}, 0.057 \mathrm{mmol})$ in anhydrous DMSO $(0.20 \mathrm{~mL})$ was added and the mixture stirred 1.25 h. A mixture of ice and saturated aqueous ammonium chloride $(1: 1,10 \mathrm{~mL})$ was added followed by ether $(10 \mathrm{~mL})$. The aqueous phase was extracted with ether $(3 \times 10$ $\mathrm{mL}$ ) and the organic extracts were filtered through Celite ${ }^{\circledR}$ using ether $(20 \mathrm{~mL})$. The filtrate was washed with brine $(50 \mathrm{~mL})$, dried $\left(\mathrm{MgSO}_{4}\right)$ and concentrated under reduced pressure. Chromatography of the residue using base-washed silica (0.5:99.5 to 1:99 ether:light petroleum) gave the title compound $30(8 \mathrm{mg}, 0.024 \mathrm{mmol}, 8 \%)$ as a colourless oil, $R_{\mathrm{f}}=0.27(1: 99$ ether:light petroleum) (Found: $\mathrm{M}^{+}+\mathrm{H}, 337.2005 . \mathrm{C}_{16} \mathrm{H}_{31} \mathrm{O}_{3} \mathrm{~F}_{2} \mathrm{Si}$ requires $\mathrm{M}, 337.2005) ; v_{\max } / \mathrm{cm}^{-1} 2958,2929,2857,1768,1464$, $1372,1292,1255,1077,1006,837$ and $775 ; \delta_{\mathrm{H}}\left(500 \mathrm{MHz}_{2} \mathrm{CDCl}_{3}\right)$ $0.05\left(6 \mathrm{H}, \mathrm{s}, 2 \times \mathrm{SiCH}_{3}\right), 0.89\left[9 \mathrm{H}, \mathrm{s}, \mathrm{SiC}\left(\mathrm{CH}_{3}\right)_{3}\right], 0.90(3 \mathrm{H}, \mathrm{t}, J$ 7.0, 7- $\left.\mathrm{H}_{3}\right), 1.35\left(3 \mathrm{H}, \mathrm{t}, J\right.$ 7.0, $\left.\mathrm{OCH}_{2} \mathrm{CH}_{3}\right), 1.40-1.56\left(2 \mathrm{H}, \mathrm{m}, 6-\mathrm{H}_{2}\right), 2.31$ $\left(2 \mathrm{H}, \mathrm{d}, J\right.$ J 6.2, 4- $\left.\mathrm{H}_{2}\right), 3.80(1 \mathrm{H}$, pent, J 6.2, 5-H), $4.33(2 \mathrm{H}, \mathrm{q}, J$ 7.0, $\left.\mathrm{OCH}_{2}\right)$ and 5.40 and 5.60 (each $\left.1 \mathrm{H}, \mathrm{t}, J 1.9,3-\mathrm{CH}\right) ; \delta_{\mathrm{C}}(125 \mathrm{MHz}$, $\mathrm{CDCl}_{3}$ ) $-4.6,-4.5,9.2,13.9,18.0,25.9,29.4,37.7,63.0,71.5$, $114.0\left(\mathrm{t},{ }^{1} J_{\mathrm{C}-\mathrm{F}} 252.1\right), 120.5\left(\mathrm{t},{ }^{3} J_{\mathrm{C}-\mathrm{F}} 8.2\right), 137.7\left(\mathrm{t},{ }^{2} J_{\mathrm{C}-\mathrm{F}} 22.8\right)$ and $163.9\left(\mathrm{t},{ }^{2} J_{\mathrm{C}-\mathrm{F}} 34.6\right) ; \delta_{\mathrm{F}}\left(471 \mathrm{MHz}, \mathrm{CDCl}_{3}\right)-106.99$ and -105.25 (both $\left.\mathrm{d}, J_{\mathrm{F}-\mathrm{F}} 253.4\right) ; \mathrm{m} / \mathrm{e}\left(\mathrm{ES}^{+}\right) 359.2\left(\mathrm{M}^{+}+23,50 \%\right)$ and 210.2 (100). This capricious reaction required copper powder that was very fine, bronze-pink in colour and extremely lustrous, anhydrous solvent and vigorous stirring, to generate the active copper-reagent species.

This procedure gave ethyl (E)-2,2-difluoro-5-tertbutyldimethylsilyloxy-3-(tert-butyldimethylsilyloxymethyl)pent3-enoate 33 ( $3 \mathrm{mg}, 0.007 \mathrm{mmol}, 12 \%$ ) as a colourless oil (Found: $\mathrm{M}^{+}+\mathrm{H}, 439.2510 . \mathrm{C}_{20} \mathrm{H}_{41} \mathrm{O}_{4} \mathrm{~F}_{2} \mathrm{Si}_{2}$ requires $\left.\mathrm{M}, 439.2511\right) ; v_{\max } / \mathrm{cm}^{-1}$ 2954, 2931, 2886, 2858, 1769, 1472, 1255, 1095, 836 and 778; $\delta_{\mathrm{H}}\left(400 \mathrm{MHz}, \mathrm{CDCl}_{3}\right) 0.07$ and 0.09 (each $\left.6 \mathrm{H}, \mathrm{s}, 2 \times \mathrm{SiCH}_{3}\right), 0.89$ and 0.91 [each $9 \mathrm{H}, \mathrm{s}, \mathrm{SiC}\left(\mathrm{CH}_{3}\right)_{3}$ ], $1.34\left(3 \mathrm{H}, \mathrm{t}, J 7.3, \mathrm{OCH}_{2} \mathrm{CH}_{3}\right)$, $4.29\left(2 \mathrm{H}, \mathrm{s}, 3-\mathrm{CH}_{2}\right), 4.29\left(2 \mathrm{H}, \mathrm{q}, J\right.$ 7.3, $\left.\mathrm{OCH}_{2} \mathrm{CH}_{3}\right), 4.39-4.42(2 \mathrm{H}$, $\left.\mathrm{m}, 5-\mathrm{H}_{2}\right)$ and $6.39(1 \mathrm{H}, \mathrm{t}, J 6.3,4-\mathrm{H}) ; \delta_{\mathrm{C}}\left(100 \mathrm{MHz}^{\mathrm{C}} \mathrm{CDCl}_{3}\right)-5.7$, $-5.3,13.9,18.3(2), 25.8,25.9,57.4,59.7,62.7,113.0\left(t,{ }^{1} J_{C-F}\right.$ 251.1), $130.6\left(t,{ }^{2} J_{C-F} 21.4\right), 135.6\left(t,{ }^{3} J_{C-F} 8.8\right)$ and $163.6\left(t,{ }^{2} J_{C-F}\right.$ 34.6); $\delta_{\mathrm{F}}\left(377 \mathrm{MHz}, \mathrm{CDCl}_{3}\right.$ ) $-106.47 ; \mathrm{m} / \mathrm{z}\left(\mathrm{ES}^{+}\right) 461.3\left(\mathrm{M}^{+}+23\right.$, $100 \%)$.

This procedure gave ethyl (E)-2,2-difluoro-5-tertbutyldiphenylsilyloxy-3-(tert-butyldiphenylsilyloxymethyl)pent3-enoate 34 ( $4 \mathrm{mg}, 0.006 \mathrm{mmol}, 14 \%)$ as a colourless oil, $R_{\mathrm{f}}=$ 0.39 (5:95 ether:light petroleum); $v_{\max } / \mathrm{cm}^{-1} 3072,2957,2930$, $2857,1769,1472,1428,1290,1112,1022,844,740$ and $702 ; \delta_{H}$ $\left(400 \mathrm{MHz}, \mathrm{CDCl}_{3}\right) 0.92$ and 1.01 [each $9 \mathrm{H}, \mathrm{s}, \mathrm{SiC}\left(\mathrm{CH}_{3}\right)_{3}$ ], $1.29(3$ $\left.\mathrm{H}, \mathrm{t}, J \mathrm{~J} .0, \mathrm{OCH}_{2} \mathrm{CH}_{3}\right), 4.02\left(2 \mathrm{H}, \mathrm{s}, 3-\mathrm{CH}_{2}\right), 4.21-4.28\left(4 \mathrm{H}, \mathrm{m}, 5-\mathrm{H}_{2}\right.$, $\left.\mathrm{OCH}_{2} \mathrm{CH}_{3}\right), 6.32(1 \mathrm{H}, \mathrm{t}, J \mathrm{~J} .6,4-\mathrm{H}), 7.26-7.44(12 \mathrm{H}, \mathrm{m}, \mathrm{ArH})$ and 7.53 and 7.59 (each $4 \mathrm{H}, \mathrm{dd}, J$ 8.0, 1.5, $\mathrm{ArH}) ; \delta_{\mathrm{F}}\left(377 \mathrm{MHz}, \mathrm{CDCl}_{3}\right)$ -105.74; $m / z\left(\mathrm{ES}^{+}\right) 704.5\left(\mathrm{M}^{+}+18,100 \%\right)$.

1-tert-Butyldiphenylsilyloxy-4,4-difluorohex-5-en-3-ol (40). 3-Bromo-3,3-difluoroprop-1-ene $37(0.5 \mathrm{~g}, 3.18 \mathrm{mmol})$ was added to a vigorously stirred suspension of indium powder $(0.37$ $\mathrm{g}, 3.18 \mathrm{mmol})$ in deionised water $(6.3 \mathrm{~mL})$ and the mixture was stirred for $10 \mathrm{~min}$. The aldehyde $39(0.70 \mathrm{~g}, 2.23 \mathrm{mmol})$ in THF $(4.40 \mathrm{~mL})$ was added and the mixture stirred for $5 \mathrm{~h}$. Aqueous hydrogen chloride $(10 \%, 50 \mathrm{~mL})$ and EtOAc $(50 \mathrm{~mL})$ were added and the aqueous phase was extracted with EtOAc $(3 \times 50 \mathrm{~mL})$. The organic extracts were washed with brine $(200 \mathrm{~mL})$, dried $\left(\mathrm{MgSO}_{4}\right)$ and concentrated under reduced pressure. Chromatography of the residue (10:90 ether:light petroleum) gave the title compound $40(0.84 \mathrm{~g}, 2.16 \mathrm{mmol}, 97 \%)$ as a colourless oil, $R_{\mathrm{f}}=0.78$ (50:50 ether:light petroleum) (Found: $\mathrm{M}^{+}$ 
$+\mathrm{Na}, 413.1715 . \mathrm{C}_{22} \mathrm{H}_{28} \mathrm{O}_{2} \mathrm{~F}_{2} \mathrm{SiNa}$ requires $\left.\mathrm{M}, 413.1724\right) ; v_{\max } / \mathrm{cm}^{-1}$ $3435,3071,2958,2931,2889,2857,1472,1427,1111,822,738$ and $701 ; \delta_{\mathrm{H}}\left(500 \mathrm{MHz}, \mathrm{CDCl}_{3}\right) 1.12\left[9 \mathrm{H}, \mathrm{s}, \mathrm{SiC}\left(\mathrm{CH}_{3}\right)_{3}\right], 1.81$ and 1.92 (each $1 \mathrm{H}, \mathrm{m}, 2-\mathrm{H}), 3.21(1 \mathrm{H}, \mathrm{br} . \mathrm{s}, \mathrm{OH}), 3.92$ and 4.00 (each $1 \mathrm{H}, \mathrm{m}, 1-\mathrm{H}), 4.16(1 \mathrm{H}, \mathrm{q}, \mathrm{J}$ 10.1, 3-H), $5.57(1 \mathrm{H}, \mathrm{d}, \mathrm{J}$ 11.0, 6-H), $5.77\left(1 \mathrm{H}, \mathrm{d}, \mathrm{J} 17.4,6-\mathrm{H}^{\prime}\right), 6.08(1 \mathrm{H}, \mathrm{m}, 5-\mathrm{H}), 7.40-7.51(6 \mathrm{H}, \mathrm{m}$, $\mathrm{ArH})$ and 7.70-7.79 $(4 \mathrm{H}, \mathrm{m}, \mathrm{ArH}) ; \delta_{\mathrm{C}}\left(125 \mathrm{MHz}_{\mathrm{CDCl}}\right)$ 19.0, 26.8, $31.8,62.0,72.6\left(t,{ }^{2} J_{C-F} 30.0\right), 119.8\left(t,{ }^{1} J_{C-F} 243.9\right), 120.8\left(t,{ }^{3} J_{C-F}\right.$ 10.0), 127.8, 129.9, 130.1 (t, $\left.{ }^{2} J_{\text {C-F }} 25.5\right), 132.8,132.9$ and 135.5; $\delta_{\mathrm{F}}\left(470 \mathrm{MHz}, \mathrm{CDCl}_{3}\right)-113.58\left(\mathrm{~d},{ }^{2} J_{\mathrm{F} F \mathrm{~F}} 247.6\right)$ and $-108.24\left(\mathrm{~d},{ }^{2} J_{\mathrm{F}-\mathrm{F}}\right.$ 249.4); $m / z\left(\mathrm{ES}^{+}\right) 413.2\left(\mathrm{M}^{+}+23,100 \%\right)$.

\section{1,3-Bis(tert-butyldiphenylsilyloxy)-4,4-difluorohex-5-ene} (41). 3-Bromo-3,3-difluoroprop-1-ene 37 (0.177 mL, $1.74 \mathrm{mmol}$ ) was added to a vigorously stirred suspension of indium powder $(0.2 \mathrm{~g}, 1.74 \mathrm{mmol})$ and lithium iodide $(0.23 \mathrm{~g}, 1.74 \mathrm{mmol})$ in deionised water $(3.46 \mathrm{~mL})$ and the mixture stirred for $10 \mathrm{~min}$. The aldehyde $39(0.27 \mathrm{~g}, 0.867 \mathrm{mmol})$ in THF $(1.73 \mathrm{~mL})$ was added and the reaction mixture stirred for $72 \mathrm{~h}$. Aqueous hydrogen chloride $(10 \%, 30 \mathrm{~mL})$ and EtOAc $(10 \mathrm{~mL})$ were added and the aqueous phase was extracted with EtOAc $(3 \times 30 \mathrm{~mL})$. The organic extracts were washed with brine $(100 \mathrm{~mL})$, dried $\left(\mathrm{Na}_{2} \mathrm{SO}_{4}\right)$ and concentrated under reduced pressure to give a yellow oil $(0.375 \mathrm{~g})$ that was azeotroped with benzene before being taken into DCM $(6.0 \mathrm{~mL})$. Imidazole $(0.142 \mathrm{~g}, 2.08 \mathrm{mmol})$ and tert-butyldiphenylsilyl chloride $(0.27 \mathrm{~mL}, 1.04 \mathrm{mmol})$ were added at $0{ }^{\circ} \mathrm{C}$ and the reaction mixture was stirred at $\mathrm{rt}$ for $48 \mathrm{~h}$. Saturated aqueous ammonium chloride $(30 \mathrm{~mL})$ and DCM (30 $\mathrm{mL}$ ) were added and the aqueous phase was extracted into DCM $(3 \times 30 \mathrm{~mL})$. The organic extracts were washed with brine (120 $\mathrm{mL})$, dried $\left(\mathrm{MgSO}_{4}\right)$ and concentrated under reduced pressure. Chromatography of the residue (1:99 ether:light petroleum) gave the title compound 41 (0.39 g, $0.622 \mathrm{mmol}, 72 \%)$ as a colourless oil, $R_{\mathrm{f}}=0.15$ (1:99 ether:light petroleum) (Found: $\mathrm{M}^{+}$ $+\mathrm{Na}, 651.2896 . \mathrm{C}_{38} \mathrm{H}_{46} \mathrm{O}_{2} \mathrm{~F}_{2} \mathrm{Si}_{2} \mathrm{Na}$ requires $\left.\mathrm{M}, 651.2897\right) ; v_{\max } / \mathrm{cm}$ ${ }^{1}$ 3072, 2959, 2931, 2887, 2858, 1473, 1428, 1112, 822, 740 and $701 ; \delta_{\mathrm{H}}\left(400 \mathrm{MHz}, \mathrm{CDCl}_{3}\right) 0.93$ and 1.03 [each $9 \mathrm{H}, \mathrm{s}, \mathrm{SiC}\left(\mathrm{CH}_{3}\right)_{3}$ ], 1.72-1.86 $\left(2 \mathrm{H}, \mathrm{m}, 2-\mathrm{H}_{2}\right), 3.46$ and 3.53 (each $\left.1 \mathrm{H}, \mathrm{m}, 1-\mathrm{H}\right), 4.03$ $(1 \mathrm{H}, \mathrm{m}, 3-\mathrm{H}), 5.47\left(1 \mathrm{H}, \mathrm{d}, J\right.$ 11.1, 6-H), $5.61\left(1 \mathrm{H}, \mathrm{d}, J\right.$ 17.4, 6- $\left.\mathrm{H}^{\prime}\right)$, $5.98(1 \mathrm{H}, \mathrm{m}, 5-\mathrm{H}), 7.29-7.35$ and 7.36-7.44 (each $6 \mathrm{H}, \mathrm{m}, \mathrm{ArH}$ ) and 7.45-7.51 and 7.61-7.66 (each $4 \mathrm{H}, \mathrm{m}, \mathrm{ArH}) ; \delta_{\mathrm{c}}(100 \mathrm{MHz}$, $\mathrm{CDCl}_{3}$ ) 18.7, 19.3, 26.4, 26.6, 35.1, 59.9, 71.8 (dd, ${ }^{2} J_{\mathrm{C}-\mathrm{F}} 32.4$, 26.9), $119.9\left(\mathrm{t},{ }^{1} J_{\mathrm{C}-\mathrm{F}} 244.6\right), 120.4\left(\mathrm{t},{ }^{3} J_{\mathrm{C}-\mathrm{F}} 9.3\right), 127.2(2), 129.1$, $129.2,129.3,129.4,129.7\left(t,{ }^{2} J_{C-F} 26.0\right), 132.0,133.2,133.3(2)$, 135.1, 135.2, 135.6 and 135.8; $\delta_{\mathrm{F}}\left(377 \mathrm{MHz}, \mathrm{CDCl}_{3}\right)-109.55$ and -103.70 (both d, ${ }^{2} J_{\mathrm{F}-\mathrm{F}} 247.3$ ); $\mathrm{m} / \mathrm{z}\left(\mathrm{ES}^{+}\right) 651.4\left(\mathrm{M}^{+}+23,100 \%\right)$.

\section{Methyl}

2,2-difluoro-3,5-bis(tert-

butyldiphenylsilyloxy)pentanoate (42). A stream of ozone was bubbled through a solution of alkene $41(0.34 \mathrm{~g}, 0.54 \mathrm{mmol})$ in dry DCM $(6.55 \mathrm{~mL})$ at $-78{ }^{\circ} \mathrm{C}$ for $5 \mathrm{~min}$ until a blue colour persisted. The system was purged with oxygen for $10 \mathrm{~min}$, dimethyl sulfide $(0.20 \mathrm{~mL}, 2.72 \mathrm{mmol})$ was added and the mixture was stirred at $\mathrm{rt}$ for $3.5 \mathrm{~h}$. After concentration under reduced pressure, the residue was dissolved in ${ }^{t} \mathrm{BuOH}(9.5 \mathrm{~mL})$ and 2-methylbut-2-ene ( $2 \mathrm{M}$ in THF, $0.63 \mathrm{~mL}$ ) was added followed by a solution of sodium chlorite $(0.295 \mathrm{~g}, 3.26 \mathrm{mmol})$ and $\mathrm{Na}_{2} \mathrm{H}_{2} \mathrm{PO}_{4} .2 \mathrm{H}_{2} \mathrm{O}(0.85 \mathrm{~g}, 5.44 \mathrm{mmol})$ in distilled water $(4.85$ $\mathrm{mL})$. After stirring for $16 \mathrm{~h}$, brine $(10 \mathrm{~mL})$ and EtOAc $(10 \mathrm{~mL})$ were added and the aqueous phase was extracted with EtOAc ( 3 $\times 10 \mathrm{~mL}$ ). The organic extracts were washed with brine $(40 \mathrm{~mL})$, dried $\left(\mathrm{MgSO}_{4}\right)$ and concentrated under reduced pressure to give an oil $(0.39 \mathrm{~g}), R_{\mathrm{f}}=0.15$ (1:10 ether:light petroleum). The oil was dissolved in anhydrous toluene $(4.4 \mathrm{~mL})$ and methanol $(1.1 \mathrm{~mL})$ and trimethylsilyl diazomethane $(2 \mathrm{M}$ in hexanes, $0.33 \mathrm{~mL}, 0.66$ mmol) was added dropwise before stirring for $48 \mathrm{~h}$. After concentration under reduced pressure, chromatography of the residue (0.5:99.5 to $1: 99$ ether:light petroleum) gave the title compound $42(0.29 \mathrm{~g}, 0.436 \mathrm{mmol}, 80 \%)$ as a colourless oil, $R_{\mathrm{f}}=$ 0.40 (10:90 ether:light petroleum) (Found: $\mathrm{M}^{+}+\mathrm{NH}_{4}, 678.3253$. $\mathrm{C}_{38} \mathrm{H}_{50} \mathrm{NO}_{4} \mathrm{~F}_{2} \mathrm{Si}_{2}$ requires $\left.\mathrm{M}, 678.3241\right) ; v_{\text {max }} / \mathrm{cm}^{-1} 2957,2932$, $2858,1764,1473,1427,1105,821$ and $734 ; \delta_{H}\left(400 \mathrm{MHz}, \mathrm{CDCl}_{3}\right)$ 0.93 and 1.01 [each $9 \mathrm{H}, \mathrm{s}, \mathrm{SiC}\left(\mathrm{CH}_{3}\right)_{3}$ ], 1.89 and 1.96 (each $1 \mathrm{H}$, $\mathrm{dq}, J$ 13.0, 6.5, 4-H), 3.42 and 3.51 (each $1 \mathrm{H}, \mathrm{dt}, J$ 10.5, 6.5, 5-H), $3.77\left(3 \mathrm{H}, \mathrm{s}, \mathrm{OCH}_{3}\right), 4.40(1 \mathrm{H}, \mathrm{m}, 3-\mathrm{H}), 7.29-7.44(12 \mathrm{H}, \mathrm{m}, \mathrm{ArH})$ and 7.45-7.50 and 7.61-7.68 (each $4 \mathrm{H}, \mathrm{m}, \mathrm{ArH}) ; \delta_{\mathrm{C}}(125 \mathrm{MHz}$, $\mathrm{CDCl}_{3}$ ) 19.0, 19.4, 26.7(2), 34.5, 53.0, 59.8, 70.7 ( $\left(\mathrm{t}^{2} \mathrm{~J}_{\mathrm{C}-\mathrm{F}} 25.5\right.$ ), $115.2\left(t,{ }^{1} J_{C-F} 253.9\right), 127.5,127.7,129.5(2), 129.8,129.9,132.1$, $133.1,133.6(2), 135.4,135.5,135.8,136.0$ and $164.0\left(t,{ }^{2} J_{C-F}\right.$ 31.9); $\delta_{\mathrm{F}}\left(377 \mathrm{MHz}, \mathrm{CDCl}_{3}\right)-117.95$ and -111.75 (both d, ${ }^{2} J_{\mathrm{F}-\mathrm{F}}$ 258.9); $m / z\left(\mathrm{ES}^{+}\right) 683.5\left(\mathrm{M}^{+}+23,100 \%\right)$.

\section{5,7-Bis(tert-butyldiphenylsilyloxy)-4,4-difluoro-2,2-}

dimethylheptan-3-one (43). tert-Butyllithium (1.7 $\mathrm{M}$ in pentane, $0.07 \mathrm{~mL}, 0.119 \mathrm{mmol}$ ) was added to the methyl ester $42(74 \mathrm{mg}$, $0.112 \mathrm{mmol})$ in dry THF $(2.2 \mathrm{~mL})$ at $-78{ }^{\circ} \mathrm{C}$ and the solution stirred at $-78{ }^{\circ} \mathrm{C}$ for $5 \mathrm{~h}$. Saturated methanolic ammonium chloride $(5 \mathrm{~mL})$ was added at $-78{ }^{\circ} \mathrm{C}$ and the mixture was allowed to warm to rt. Water $(10 \mathrm{~mL})$ and $\mathrm{DCM}(10 \mathrm{~mL})$ were added and the aqueous phase was extracted with DCM $(3 \times 10$ $\mathrm{mL})$. The organic extracts were washed with brine $(40 \mathrm{~mL})$, dried $\left(\mathrm{MgSO}_{4}\right)$ and concentrated under reduced pressure. Chromatograpy of the residue (0.5:99.5 ether:light petroleum) gave the title compound 43 (40 $\mathrm{mg}, 0.058 \mathrm{mmol}, 52 \%$ ) as a colourless oil, $R_{\mathrm{f}}=0.24$ (1:99 ether:light petroleum) (Found: $\mathrm{M}^{+}$ $+\mathrm{NH}_{4}, 704.3771 . \mathrm{C}_{41} \mathrm{H}_{56} \mathrm{NO}_{3} \mathrm{~F}_{2} \mathrm{Si}_{2}$ requires $\left.\mathrm{M}, 704.3761\right) ; v_{\text {max }} / \mathrm{cm}^{-}$ ${ }^{1}$ 3071, 2959, 2932, 2890, 2858, 1724, 1473, 1427, 1106, 1032, 822 and 739; $\delta_{\mathrm{H}}\left(400 \mathrm{MHz}, \mathrm{CDCl}_{3}\right) 0.93$ and 0.99 [each $9 \mathrm{H}, \mathrm{s}$, $\left.\mathrm{SiC}\left(\mathrm{CH}_{3}\right)_{3}\right], 1.21\left[9 \mathrm{H}, \mathrm{s}, \mathrm{C}\left(\mathrm{CH}_{3}\right)_{3}\right], 1.72-1.87\left(2 \mathrm{H}, \mathrm{m}, 6-\mathrm{H}_{2}\right), 3.43-$ $3.49\left(2 \mathrm{H}, \mathrm{m}, 7-\mathrm{H}_{2}\right), 4.54(1 \mathrm{H}, \mathrm{m}, 5-\mathrm{H}), 7.28-7.43(12 \mathrm{H}, \mathrm{m}, \mathrm{ArH})$ and 7.43-7.50 and 7.66-7.71 (each $4 \mathrm{H}, \mathrm{m}, \mathrm{ArH}) ; \delta_{\mathrm{c}}(100 \mathrm{MHz}$, $\left.\mathrm{CDCl}_{3}\right)$ 19.0, 19.6, 25.9, 26.7, 26.8, 34.3, 43.7, 60.0, $69.7\left(\mathrm{t},{ }^{2} J_{\mathrm{C}-\mathrm{F}}\right.$ $25.8), 118.2\left(t,{ }^{1} J_{C-F} 261.7\right), 127.5,127.6,129.4,129.5,129.7$, $129.8,132.2,133.5,133.6,133.7,135.4,135.5,135.9,136.0$ and $204.6\left(\mathrm{t},{ }^{2} J_{\mathrm{C}-\mathrm{F}} 27.2\right) ; \delta_{\mathrm{F}}\left(377 \mathrm{MHz}, \mathrm{CDCl}_{3}\right)-111.70$ and -109.97 (both $\left.d,{ }^{2} J_{F-F} 280.4\right) ; m / z\left(E S^{+}\right) 709.6\left(\mathrm{M}^{+}+23,100 \%\right)$.

\section{(2RS,4RS)-2-tert-Butyl-3,3-difluoro-2,4-} dihydroxytetrahydro-2H-pyran (44). Tetra- $n$-butylammonium fluoride ( $1 \mathrm{M}$ in THF, $0.70 \mathrm{~mL}, 0.704 \mathrm{mmol}$ ) was added to the ketone $43(0.23 \mathrm{~g}, 0.335 \mathrm{mmol})$ in THF $(3.35 \mathrm{~mL})$ at $0{ }^{\circ} \mathrm{C}$ and the solution stirred at rt for $15 \mathrm{~h}$. Saturated aqueous ammonium chloride $(10 \mathrm{~mL})$, water $(10 \mathrm{~mL})$ and EtOAc $(20 \mathrm{~mL})$ were added and the aqueous phase was extracted into EtOAc $(3 \times 20 \mathrm{~mL})$. 
The organic extracts were washed with brine $(60 \mathrm{~mL})$, dried $\left(\mathrm{MgSO}_{4}\right)$ and concentrated under reduced pressure. Chromatography of the residue (10:90 ether:light petroleum to ether) gave a mixture $\left({ }^{1} \mathrm{H} N \mathrm{NMR}\right)$ of the hemiacetals $\mathbf{4 4}$ and $\mathbf{4 5}$ (62 mg). Recrystallisation from toluene gave the title compound 44 (53 mg, $0.25 \mathrm{mmol}, 75 \%$ ) as white needles, $R_{\mathrm{f}}=0.58$ (ether), m.p. $=91-94{ }^{\circ} \mathrm{C}$ (Found: $\mathrm{C}, 51.39 ; \mathrm{H}, 7.64 . \mathrm{C}_{9} \mathrm{H}_{16} \mathrm{O}_{3} \mathrm{~F}_{2}$ requires $\mathrm{C}$, 51.40; $\mathrm{H}, 7.70 \%$; Found: $\mathrm{M}^{+}+\mathrm{Na}, 233.0956$. $\mathrm{C}_{9} \mathrm{H}_{16} \mathrm{O}_{3} \mathrm{~F}_{2} \mathrm{Na}$ requires $M, 233.0965) ; v_{\max } / \mathrm{cm}^{-1} 3434,2965,2941,1338,1093$, 1066, 1005, 993, 906, 875 and 752; $\delta_{\mathrm{H}}\left(400 \mathrm{MHz}\right.$, benzene- $\left.d_{6}\right)$ $1.10\left[9 \mathrm{H}, \mathrm{s}, \mathrm{C}\left(\mathrm{CH}_{3}\right)_{3}\right], 1.38(1 \mathrm{H}, \mathrm{t}, J 1.5, \mathrm{OH}), 1.42(1 \mathrm{H}, \mathrm{m}, 5-\mathrm{H})$, $1.64\left(1 \mathrm{H}, \mathrm{ddq}, \mathrm{J} 2.0,5.5,13.5,5-\mathrm{H}^{\prime}\right), 1.86(1 \mathrm{H}, \mathrm{br} . \mathrm{s}, \mathrm{OH}), 3.25$ (1 $\mathrm{H}$, ddt, J 11.5, 5.3, 1.5, 6-H), $3.51\left(1 \mathrm{H}, \mathrm{m}, 6-\mathrm{H}^{\prime}\right)$ and $4.06(1 \mathrm{H}, \mathrm{m}$, $4-H) ; \delta_{C}\left(100 \mathrm{MHz}\right.$, benzene- $\left.d_{6}\right) 26.2\left(\mathrm{t},{ }^{4} J_{\mathrm{C}-\mathrm{F}} 3.6\right), 32.3\left(\mathrm{~d},{ }^{3} J_{\mathrm{C}-\mathrm{F}}\right.$ 5.8), $40.0\left(\mathrm{t},{ }^{3} J_{\mathrm{C}-\mathrm{F}} 2.9\right), 58.7,68.0\left(\mathrm{dd},{ }^{2} J_{\mathrm{C}-\mathrm{F}} 22.6,21.1\right), 99.7(\mathrm{dd}$, $\left.{ }^{2} J_{\mathrm{C}-\mathrm{F}} 29.9,24.1\right)$ and $121.3\left(\mathrm{dd},{ }^{1} J_{\mathrm{C}-\mathrm{F}} 258.0,251.4\right) ; \delta_{\mathrm{F}}(377 \mathrm{MHz}$, benzene- $\left.d_{6}\right)-130.11\left(\mathrm{~d},{ }^{2} J_{\mathrm{F}-\mathrm{F}} 240.0\right)$ and $-118.89\left(\mathrm{~d},{ }^{2} J_{\mathrm{F}-\mathrm{F}} 238.7\right)$; $\mathrm{m} / \mathrm{z}\left(\mathrm{ES}^{-}\right) 209$ ([M-H]', 100\%); $\left(\mathrm{ES}^{+}\right) 233.1\left(\mathrm{M}^{+}+23,100 \%\right)$.

After standing in solution in benzene- $d_{6}$ for $48 \mathrm{~h}$, the hemiacetal 44 equilibrated with the hemiacetal $45\left({ }^{1} \mathrm{H} N M R\right)$, 45:44 = 60:40 ( $\left.{ }^{19} \mathrm{~F} \mathrm{NMR}\right) ; \delta_{\mathrm{H}}\left(400 \mathrm{MHz}\right.$, benzene- $\left.d_{6}\right)$ hemiacetal $451.15(1 \mathrm{H}, \mathrm{m}, 5-\mathrm{H}), 1.37\left[9 \mathrm{H}, \mathrm{s}, \mathrm{C}\left(\mathrm{CH}_{3}\right)_{3}\right], 1.75\left(1 \mathrm{H}, \mathrm{m}, 5-\mathrm{H}^{\prime}\right)$, $2.05(1 \mathrm{H}$, br. s, OH), 3.30 and 3.56 (each $1 \mathrm{H}, \mathrm{m}, 6-\mathrm{H}$ ) and 3.93 $(1 \mathrm{H}, \mathrm{t}, J 12.5,4-\mathrm{H}) ; \delta_{\mathrm{C}}\left(100 \mathrm{MHz}\right.$, benzene- $\left.d_{6}\right) 26.5\left(\mathrm{t},{ }^{4} J_{\mathrm{C}-\mathrm{F}} 2.9\right)$, $30.7\left(d,{ }^{3} J_{C-F} 5.1\right), 40.4\left(t,{ }^{3} J_{C-F} 2.2\right), 54.5,70.2\left(d d,{ }^{2} J_{C-F} 34.3,24.1\right)$, $100.5\left(d d,{ }^{2} J_{C-F} 26.2,24.8\right)$ and $117.6\left(d d,{ }^{1} J_{C-F} 260.2,253.7\right) ; \delta_{F}$ (377 MHz, benzene- $d_{6}$ ) -115.73 (d, ${ }^{2} J_{\mathrm{F}-\mathrm{F}} 252.3$ ) and $-113.93(\mathrm{~d}$, ${ }^{2} J_{\mathrm{F}-\mathrm{F}}$ 253.7). Attempts to separate this mixture by chromatography were unsuccessful.

\section{6,8-Bis(tert-butyldiphenylsilyloxy)-5,5-difluoro-3,3-}

dimethyloct-1-en-4-one (46). 3-Methylbut-2-enylmagnesium chloride (0.31 $\mathrm{M}$ in THF, $1.22 \mathrm{~mL}, 0.38 \mathrm{mmol}$ ) was added dropwise to the methyl ester $42(0.167 \mathrm{~g}, 0.253 \mathrm{mmol})$ in dry THF $(2.7 \mathrm{~mL})$ at $-10{ }^{\circ} \mathrm{C}$ and the solution stirred at $-10{ }^{\circ} \mathrm{C}$ for 12 h. Saturated methanolic ammonium chloride $(2.5 \mathrm{~mL})$ was added at $-10{ }^{\circ} \mathrm{C}$ and the mixture was allowed to warm to $\mathrm{rt}$. Water $(10 \mathrm{~mL})$ and EtOAc $(10 \mathrm{~mL})$ were added and the aqueous phase was extracted with EtOAc $(3 \times 10 \mathrm{~mL})$ The organic extracts were washed with brine $(40 \mathrm{~mL})$, dried $\left(\mathrm{MgSO}_{4}\right)$ and concentrated under reduced pressure. Chromatography of the residue (0.5:99.5 ether:light petroleum) gave the title compound $46(0.12 \mathrm{~g}, 0.17 \mathrm{mmol}, 66 \%)$ as a colourless oil, $R_{\mathrm{f}}=0.58(10: 90$ ether:light petroleum) (Found: $\mathrm{M}^{+}+\mathrm{NH}_{4}, 716.3761$. $\mathrm{C}_{42} \mathrm{H}_{56} \mathrm{NO}_{3} \mathrm{~F}_{2} \mathrm{Si}_{2}$ requires $\left.\mathrm{M}, 716.3761\right) ; v_{\max } / \mathrm{cm}^{-1} 2959,2931$, 2890, 2858, 1726, 1472, 1427, 1390, 1362, 1106, 822 and 739; $\delta_{\mathrm{H}}\left(500 \mathrm{MHz}, \mathrm{CDCl}_{3}\right) 0.92$ and 0.98 [each $9 \mathrm{H}, \mathrm{s}, \mathrm{SiC}\left(\mathrm{CH}_{3}\right)_{3}$ ], 1.28 and 1.29 (each $\left.3 \mathrm{H}, \mathrm{s}, 3-\mathrm{CH}_{3}\right), 1.70-1.83\left(2 \mathrm{H}, \mathrm{m}, 7-\mathrm{H}_{2}\right), 3.42-3.50$ $\left(2 \mathrm{H}, \mathrm{m}, 8-\mathrm{H}_{2}\right), 4.47(1 \mathrm{H}, \mathrm{m}, 6-\mathrm{H}), 5.09-5.12\left(2 \mathrm{H}, \mathrm{m}, 1-\mathrm{H}_{2}\right), 5.94$ $(1 \mathrm{H}, \mathrm{dd}, \mathrm{J} 17.3,10.4,2-\mathrm{H}), 7.29-7.44(12 \mathrm{H}, \mathrm{m}, \mathrm{ArH})$ and 7.447.49 and 7.65-7.69 (each $4 \mathrm{H}, \mathrm{m}, \mathrm{ArH}) ; \delta_{\mathrm{C}}\left(125 \mathrm{MHz}, \mathrm{CDCl}_{3}\right)$ 19.0, 19.6, 23.3, 23.8, 26.7, 26.8, 34.4, 49.7, 60.0, $69.8\left(\mathrm{t},{ }^{2} \mathrm{~J}_{\mathrm{C}-\mathrm{F}} 24.4\right)$, $115.5,118.0$ (t, $\left.{ }^{1} J_{\mathrm{C}-\mathrm{F}} 260.0\right), 127.5,127.6,129.4,129.5,129.7$, 129.8, 132.3, 133.5, 133.7(2), 135.4, 135.5, 135.9, 136.0, 139.7 and $201.7\left(\mathrm{t},{ }^{2} J_{\mathrm{C}-\mathrm{F}} 28.0\right) ; \delta_{\mathrm{F}}\left(471 \mathrm{MHz}, \mathrm{CDCl}_{3}\right)-110.70$ and -109.98 (both d, $\left.{ }^{2} J_{F-F} 277.7\right) ; m / z\left(E S^{+}\right) 716.8\left(\mathrm{M}^{+}+18,30 \%\right)$.
(2RS,4RS)-3,3-Difluoro-2,4-dihydroxy-2-(2-methylbut-3-en2-yl)tetrahydro-2H-pyran (47). Tetra- $n$-butylammonium fluoride ( $1 \mathrm{M}$ in THF, $0.81 \mathrm{~mL}, 0.81 \mathrm{mmol}$ ) was added to the ketone $46(0.27 \mathrm{~g}, 0.386 \mathrm{mmol})$ in THF $(3.96 \mathrm{~mL})$ at $0{ }^{\circ} \mathrm{C}$ and the solution stirred at $\mathrm{rt}$ for $15 \mathrm{~h}$. Saturated aqueous ammonium chloride $(10 \mathrm{~mL})$, water $(10 \mathrm{~mL})$ and EtOAc $(20 \mathrm{~mL})$ were added and the aqueous phase was extracted into EtOAc $(3 \times 20 \mathrm{~mL})$. The organic extracts were washed with brine $(80 \mathrm{~mL})$, dried $\left(\mathrm{MgSO}_{4}\right)$ and concentrated under reduced pressure. Chromatography of the residue (10:90 ether:light petroleum to ether) gave a mixture ( ${ }^{1} \mathrm{H}$ NMR) of the hemiacetals $\mathbf{4 7}$ and $\mathbf{4 8}$ (83 $\mathrm{mg}$ ) as a colourless oil. Repeated trituration with pentane gave the title compound 47 ( $70 \mathrm{mg}, 0.315 \mathrm{mmol}, 82 \%$ ) as white needles, $R_{\mathrm{f}}=0.26$ (20:80 light petroleum:ether), m.p. 89-94 ${ }^{\circ} \mathrm{C}$ (Found: $\mathrm{M}^{+}+\mathrm{Na}$, 245.0974. $\mathrm{C}_{10} \mathrm{H}_{16} \mathrm{O}_{3} \mathrm{~F}_{2} \mathrm{Na}$ requires $\mathrm{M}, 245.0965$ ); $v_{\max } / \mathrm{cm}^{-1} 3436,2978,2960,2934,2899,1378,1093,1065,1001$, $945,904,873,789$ and $752 ; \delta_{H}\left(500 \mathrm{MHz}\right.$, benzene- $\left.d_{6}\right) 1.30(3 \mathrm{H}$, d, J 3.8, $\left.1^{\prime}-\mathrm{H}_{3}\right), 1.39\left(3 \mathrm{H}, \mathrm{d}, J\right.$ J 2.2, 2' $\left.-\mathrm{CH}_{3}\right), 1.46(1 \mathrm{H}, \mathrm{m}, 5-\mathrm{H}), 1.71$ $\left(1 \mathrm{H}, \mathrm{dq}, \mathrm{J} 12.5,5.5,5-\mathrm{H}^{\prime}\right), 2.56(1 \mathrm{H}, \mathrm{br} . \mathrm{s}, \mathrm{OH}), 3.28(1 \mathrm{H}, \mathrm{dd}, \mathrm{J}$ 11.4, 5.5, 6-H), $3.56\left(2 \mathrm{H}, \mathrm{m}, 6-\mathrm{H}^{\prime}, \mathrm{OH}\right), 4.09(1 \mathrm{H}, \mathrm{m}, 4-\mathrm{H}), 4.92-$ $4.95\left(2 \mathrm{H}, \mathrm{m}, 4^{\prime}-\mathrm{H}_{2}\right)$ and $6.07\left(1 \mathrm{H}, \mathrm{dd}, J \mathrm{18.0}, 10.4,3^{\prime}-\mathrm{H}\right) ; \delta_{\mathrm{C}}(100$ $\mathrm{MHz}$, benzene- $\left.d_{6}\right) 21.7\left(\mathrm{dd},{ }^{4} J_{\mathrm{C}-\mathrm{F}} 6.6,1.5\right), 24.2\left(\mathrm{~d},{ }^{4} J_{\mathrm{C}-\mathrm{F}} 6.6\right), 32.3$ (d, $\left.{ }^{3} J_{C-F} 5.8\right), 46.5$ (d, $\left.{ }^{3} J_{C-F} 2.9\right), 58.7,68.1$ (dd, $\left.{ }^{2} J_{C-F} 21.9,20.4\right)$, $98.4\left(\mathrm{dd},{ }^{2} J_{\mathrm{C}-\mathrm{F}} 30.9,24.3\right), 115.5,121.0\left(\mathrm{dd},{ }^{1} J_{\mathrm{C}-\mathrm{F}} 260.6,252.5\right)$ and 144.6; $\delta_{F}\left(377 \mathrm{MHz}\right.$, benzene- $\left.d_{6}\right)-130.10\left(\mathrm{~d},{ }^{2} J_{\mathrm{F}-\mathrm{F}} 240.0\right)$ and $-118.89\left(d^{2} J_{F-F} 238.7\right) ; m / z(E S) 291.3(100 \%)$ and 221.2 ([M $\left.\mathrm{H}]^{-}\right)$.

After standing in solution in benzene- $d_{6}$ for $48 \mathrm{~h}$, the hemiacetal 47 had equilibrated with the hemiacetal 48, 47:48= 60:40 ( $\left.{ }^{19} \mathrm{~F} \mathrm{NMR}\right) ; \delta_{\mathrm{H}}\left(400 \mathrm{MHz}\right.$, benzene- $\left.d_{6}\right)$ hemiacetal 481.38 $(1 \mathrm{H}, \mathrm{m}, 5-\mathrm{H}), 1.44$ and 1.48 (each $3 \mathrm{H}, \mathrm{s}, 1^{\prime}-\mathrm{H}_{3}$ or $\left.2^{\prime}-\mathrm{CH}_{3}\right), 1.76$ (1 $\left.\mathrm{H}, \mathrm{m}, 5-\mathrm{H}^{\prime}\right), 2.55(1 \mathrm{H}$, br. $\mathrm{s}, \mathrm{OH}$ ), 3.31 and 3.67 (each $1 \mathrm{H}, \mathrm{m}, 6-$ $\mathrm{H}), 3.96(1 \mathrm{H}, \mathrm{t}, \mathrm{J} 13.0,4-\mathrm{H}), 5.05-5.14\left(2 \mathrm{H}, \mathrm{m}, 4^{\prime}-\mathrm{H}_{2}\right)$ and $6.48(1$ $\left.H, d d, J 17.5,11.0,3^{\prime}-H\right) ; \delta_{F}\left(377 \mathrm{MHz}\right.$, benzene- $\left.d_{6}\right)-115.75$ and -114.40 (both $d,{ }^{2} J_{F-F} 252.4$ ). Attempts to separate this mixture by chromatography were unsuccessful.

2-Difluoromethylenedecan-1-ol (54). Methyllithium (1.6 M in ether, $0.37 \mathrm{~mL}, 0.60 \mathrm{mmol}$ ) was added dropwise to the ketone $52^{28}$ (68 $\left.\mathrm{mg}, 0.30 \mathrm{mmol}\right)$ and di-iodomethane $(0.048 \mathrm{~mL}$, $0.60 \mathrm{mmol})$ in dry THF $(1.8 \mathrm{~mL})$ at $-78{ }^{\circ} \mathrm{C}$ and the mixture allowed to warm to $\mathrm{rt}$ over $30 \mathrm{~min}$ then cooled to $-78^{\circ} \mathrm{C}$. tertButyllithium (1.7 M in pentane, $5.15 \mathrm{mmol}, 3.03 \mathrm{~mL}$ ) was added and the mixture was stirred at $\mathrm{rt}$ for $16 \mathrm{~h}$. Aqueous hydrogen chloride $(1 \mathrm{M}, 20 \mathrm{~mL})$ and ether $(20 \mathrm{~mL})$ were added and the aqueous phase was extracted with ether $(3 \times 20 \mathrm{~mL})$. The organic extracts were washed with brine $(80 \mathrm{~mL})$, dried $\left(\mathrm{MgSO}_{4}\right)$ and concentrated under reduced pressure. Chromatography of the residue (1:99 to 5:95 ether:light petroleum) gave the title compound 54 (94 mg, $0.46 \mathrm{mmol}, 44 \%$ ) as a colourless oil, $R_{\mathrm{f}}=$ 0.18 (10:90 ether:light petroleum) (Found: $\mathrm{M}^{+}, 206.1477$. $\mathrm{C}_{11} \mathrm{H}_{20} \mathrm{OF}_{2}$ requires $\left.\mathrm{M}, 206.1477\right) ; v_{\max } / \mathrm{cm}^{-1} 3333,2955,2925$, 2856, 1746, 1466, 1264, 1001 and 730; $\delta_{\mathrm{H}}\left(500 \mathrm{MHz}, \mathrm{CDCl}_{3}\right) 0.88$ $\left(3 \mathrm{H}, \mathrm{t}, \mathrm{J} 7.0,10-\mathrm{H}_{3}\right), 1.20-1.35\left(10 \mathrm{H}, \mathrm{m}, 5 \times \mathrm{CH}_{2}\right), 1.40-1.48(2 \mathrm{H}$, $\left.\mathrm{m}, 4-\mathrm{H}_{2}\right), 1.96(1 \mathrm{H}$, br. s, OH$), 2.09\left(2 \mathrm{H}, \mathrm{m}, 3-\mathrm{H}_{2}\right)$ and $4.14(2 \mathrm{H}$, $\left.\mathrm{s}, 1-\mathrm{H}_{2}\right) ; \delta_{\mathrm{C}}\left(125 \mathrm{MHz}, \mathrm{CDCl}_{3}\right)$ 14.0, 22.6, 24.4, 27.5, 29.2(2), 29.3, 
31.8, 57.7, $90.0\left(\mathrm{dd},{ }^{2} J_{\mathrm{C}-\mathrm{F}} 16.4,13.7\right)$ and $154.2\left(\mathrm{t},{ }^{1} J_{\mathrm{C}-\mathrm{F}} 288.5\right) ; \delta_{\mathrm{F}}$ (377 MHz, $\mathrm{CDCl}_{3}$ ) -93.14 and -92.87 (both $d,{ }^{2} J_{F-F} 48.1$ ).

1-Bromo-2-(difluoromethylene)decane (55). Dimethyl sulfide $(0.094 \mathrm{~mL}, 1.280 \mathrm{mmol})$ was added to $N$ bromosuccinimide $(0.185 \mathrm{~g}, 1.04 \mathrm{mmol})$ in dry DCM $(4.0 \mathrm{~mL})$ at 0 ${ }^{\circ} \mathrm{C}$ and the mixture stirred for $10 \mathrm{~min}$ then cooled to $-20{ }^{\circ} \mathrm{C}$. The alcohol 54 (0.143 g, $0.693 \mathrm{mmol})$ in DCM $(3 \mathrm{~mL})$ was added and the mixture stirred at $\mathrm{rt}$ for $16 \mathrm{~h}$. Saturated aqueous sodium hydrogen carbonate $(10 \mathrm{~mL})$ and DCM $(10 \mathrm{~mL})$ were added and the aqueous phase was extracted with DCM $(3 \times 10 \mathrm{~mL})$. The organic extracts were washed with brine $(40 \mathrm{~mL})$, dried $\left(\mathrm{MgSO}_{4}\right)$ and concentrated under reduced pressure. Chromatography of the residue (light petroleum) gave the title compound $\mathbf{5 5}(0.12$ g, $0.435 \mathrm{mmol}, 63 \%$ ) as a colourless oil, $R_{\mathrm{f}}=0.41$ (light petroleum); $v_{\max } / \mathrm{cm}^{-1} 2956,2925,2856,1735,1465,1290$, 1223, 1188 and 1058; $\delta_{\mathrm{H}}\left(500 \mathrm{MHz}, \mathrm{CDCl}_{3}\right) 0.90(3 \mathrm{H}, \mathrm{t}, \mathrm{J} 7.0,10-$ $\left.\mathrm{H}_{3}\right), 1.25-1.35\left(10 \mathrm{H}, \mathrm{m}, 5 \times \mathrm{CH}_{2}\right), 1.46\left(2 \mathrm{H}, \mathrm{m}, 4-\mathrm{H}_{2}\right), 2.14(2 \mathrm{H}$, $\left.\mathrm{m}, 3-\mathrm{H}_{2}\right)$ and $4.02\left(2 \mathrm{H}, \mathrm{t},{ }^{4} J_{\mathrm{H}-\mathrm{F}} 1.9,1-\mathrm{H}_{2}\right) ; \delta_{\mathrm{C}}\left(125 \mathrm{MHz}, \mathrm{CDCl}_{3}\right)$ 14.0, 22.6, 25.2, 27.1(m), $27.8\left(\mathrm{dd},{ }^{3} J_{\mathrm{C}-\mathrm{F}} 7.4,1.8\right), 29.1,29.2,29.3$, $31.8,89.0\left(\mathrm{dd},{ }^{2} J_{\mathrm{C}-\mathrm{F}} 22.0,12.9\right)$ and $153.9\left(\mathrm{t},{ }^{1} J_{\mathrm{C}-\mathrm{F}} 291.2\right) ; \delta_{\mathrm{F}}(377$ $\mathrm{MHz}, \mathrm{CDCl}_{3}$ ) -89.64 and -88.32 (both $\mathrm{d}^{2}{ }^{2} \mathrm{~F}_{\mathrm{F}-\mathrm{F}} 35.5$ ).

Following this procedure, $N$-bromosuccinimide $(63 \mathrm{mg}, 0.36$ $\mathrm{mmol}$ ) in DCM $(1.38 \mathrm{~mL})$, dimethyl sulfide $(0.032 \mathrm{~mL}, 0.44$ $\mathrm{mmol}$ ) and the alcohol 54 (49 $\mathrm{mg}, 0.237 \mathrm{mmol}$ ) in DCM (1 mL) with heating the crude product in toluene- $d_{8}(0.5 \mathrm{~mL})$ under reflux for $16 \mathrm{~h}$ gave, after chromatography (light petroleum) a mixture (57 mg, $0.212 \mathrm{mmol}, 89 \%$ ) of the title compound 55 and 2-(bromodifluoromethyl)dec-1-ene $\mathbf{5 6}$ as a colourless oil, 55:56 $=20: 80 ; v_{\max } / \mathrm{cm}^{-1} 2956,2926,2856,1738,1465,1131,1114$, 1101, 926 and 895; $\delta_{\mathrm{H}}\left(500 \mathrm{MHz}, \mathrm{CDCl}_{3}\right)$ isomer $560.90(3 \mathrm{H}, \mathrm{t}, \mathrm{J}$ 6.9, $\left.10-\mathrm{H}_{3}\right), 1.25-1.41\left(10 \mathrm{H}, \mathrm{m}, 5 \times \mathrm{CH}_{2}\right), 1.56\left(2 \mathrm{H}, \mathrm{m}, 4-\mathrm{H}_{2}\right), 2.28$ $\left(2 \mathrm{H}, \mathrm{t}, \mathrm{J} 7.9,3-\mathrm{H}_{2}\right), 5.17\left(1 \mathrm{H}, \mathrm{t},{ }^{4} J_{\mathrm{H}-\mathrm{F}} 1.6,1-\mathrm{H}\right)$ and $5.60\left(1 \mathrm{H}, \mathrm{t},{ }^{4} \mathrm{~J}_{\mathrm{H}-}\right.$ F 1.0, 1- $\left.\mathrm{H}^{\prime}\right) ; \delta_{\mathrm{C}}\left(125 \mathrm{MHz}, \mathrm{CDCl}_{3}\right) 14.1,22.7,27.4,29.2(2), 29.2$, $29.4,29.7,31.8,114.3\left(\mathrm{t},{ }^{3} J_{\mathrm{C}-\mathrm{F}} 7.4\right), 119.7\left(\mathrm{t},{ }^{1} J_{\mathrm{C}-\mathrm{F}} 306.7\right)$ and 145.4 $\left(t,{ }^{2} J_{C-F} 19.3\right) ; \delta_{\mathrm{F}}\left(377 \mathrm{MHz} \mathrm{CDCl}_{3}\right)-48.43$.

Difluorinated alcohols 57: general procedure. Indium powder (43 $\mathrm{mg}, 0.37 \mathrm{mmol}$ ) was added to a vigorously stirred solution of 1-bromo-2-(difluoromethylene)decane $55(50 \mathrm{mg}$, $0.19 \mathrm{mmol})$ in DMF $(1.0 \mathrm{~mL})$ in a $10 \mathrm{~mL}$ Schlenk tube. After 10 $\mathrm{min}$, the aldehyde $(0.41 \mathrm{mmol})$ was added and the mixture stirred for $14 \mathrm{~h}$. Aqueous hydrogen chloride $(10 \%, 10 \mathrm{~mL})$ and ether $(10 \mathrm{~mL})$ were added and the aqueous phase was extracted with ether $(3 \times 30 \mathrm{~mL})$. The organic extracts were washed with brine $(40 \mathrm{~mL})$, dried $\left(\mathrm{MgSO}_{4}\right)$ and concentrated under reduced pressure. Chromatography of the residue (light petroleum to 5:95 ether:light petroleum) gave the alcohols $\mathbf{5 7}$ as colourless oils.

2,2-Difluoro-3-methylene-1-phenylundecan-1-ol 57a. (46 mg, $0.155 \mathrm{mmol}, 84 \%), R_{\mathrm{f}}=0.14$ (10:90 ether:light petroleum) (Found: $\mathrm{M}^{+}+\mathrm{NH}_{4}, 314.2285 . \mathrm{C}_{18} \mathrm{H}_{30} \mathrm{NOF}_{2}$ requires $\mathrm{M}, 314.2290$; $v_{\max } / \mathrm{cm}^{-1} 3380,2954,2925,2855,1455,1164,1086,1060,1026$, 920 and $743 ; \delta_{\mathrm{H}}\left(500 \mathrm{MHz}, \mathrm{CDCl}_{3}\right) 0.90\left(3 \mathrm{H}, \mathrm{t}, J\right.$ 7.0, 11- $\left.\mathrm{H}_{3}\right), 1.23-$ $1.33\left(10 \mathrm{H}, \mathrm{m}, 5 \times \mathrm{CH}_{2}\right), 1.43\left(2 \mathrm{H}, \mathrm{m}, 5-\mathrm{H}_{2}\right), 1.89-2.00(2 \mathrm{H}, \mathrm{m}, 4-$ $\left.\mathrm{H}_{2}\right), 2.43\left(1 \mathrm{H}\right.$, br. s, OH), $4.93\left(1 \mathrm{H}, \mathrm{t},{ }^{3} \mathrm{~J}_{\mathrm{H}-\mathrm{F}} 11.0,1-\mathrm{H}\right), 5.18$ and 5.31 (each $1 \mathrm{H}, \mathrm{s}, 3-\mathrm{CH}), 7.33-7.38(3 \mathrm{H}, \mathrm{m}, \mathrm{ArH})$ and 7.40-7.44 (2
$\mathrm{H}, \mathrm{m}, \mathrm{ArH}) ; \delta_{\mathrm{C}}\left(100 \mathrm{MHz}, \mathrm{CDCl}_{3}\right) 14.1,22.6,27.6,29.2(2), 29.3$, $30.5\left(\mathrm{t},{ }^{3} J_{C-F} 2.9\right), 31.8,75.4\left(t,{ }^{2} J_{C-F} 30.0\right), 116.3\left(t, J_{C-F} 8.0\right), 121.2$ $\left(\mathrm{t},{ }^{1} J_{\mathrm{C}-\mathrm{F}} 247.2\right), 127.8,128.1,128.6,136.1\left(\mathrm{t},{ }^{3} \mathrm{~J}_{\mathrm{C}-\mathrm{F}} 2.2\right)$ and 142.8 $\left(\mathrm{t},{ }^{2} \mathrm{~J}_{\mathrm{C}-\mathrm{F}} 22.6\right) ; \delta_{\mathrm{F}}\left(471 \mathrm{MHz}, \mathrm{CDCl}_{3}\right)-109.53 ; \mathrm{m} / \mathrm{z}(\mathrm{APCl}) 314.2\left(\mathrm{M}^{+}\right.$ $+18,100 \%$ ).

2,2-Difluoro-3-methylene-1-(4-methoxyphenyl)undecan-1-ol 57b. (40 mg, $0.12 \mathrm{mmol}, 65 \%), R_{\mathrm{f}}=0.65$ (50:50 ether:light petroleum) (Found: $\mathrm{M}^{+}+\mathrm{NH}_{4}, 344.2390 . \mathrm{C}_{19} \mathrm{H}_{32} \mathrm{NO}_{2} \mathrm{~F}_{2}$ requires $\mathrm{M}, 344.2396) ; v_{\max } / \mathrm{cm}^{-1} 3457,2925,2855,1613,1514,1464$, $1249,1174,1062,1032,921,855$ and $794 ; \delta_{\mathrm{H}}\left(500 \mathrm{MHz}, \mathrm{CDCl}_{3}\right)$ $0.90\left(3 \mathrm{H}, \mathrm{t}, J \mathrm{~J} .0,11-\mathrm{H}_{3}\right), 1.23-1.35\left(10 \mathrm{H}, \mathrm{m}, 5 \times \mathrm{CH}_{2}\right), 1.43(2 \mathrm{H}$, m, 5- $\left.\mathrm{H}_{2}\right), 1.89-2.01\left(2 \mathrm{H}, \mathrm{m}, 4-\mathrm{H}_{2}\right), 2.48(1 \mathrm{H}, \mathrm{br} . \mathrm{s}, \mathrm{OH}), 3.82(3 \mathrm{H}$, $\left.\mathrm{s}, \mathrm{OCH}_{3}\right), 4.87\left(1 \mathrm{H}, \mathrm{t},{ }^{3} \mathrm{~J}_{\mathrm{H}-\mathrm{F}} 11.4,1-\mathrm{H}\right), 5.18$ and 5.31 (each $1 \mathrm{H}, \mathrm{s}$, $3-\mathrm{CH}), 6.89\left(2 \mathrm{H}, \mathrm{d}, J\right.$ 8.9, ArH) and $7.34(2 \mathrm{H}, \mathrm{d}, J 8.5, \mathrm{ArH}) ; \delta_{\mathrm{c}}$ $\left(100 \mathrm{MHz} \mathrm{CDCl}_{3}\right)$ 14.1, 22.6, 27.6, 29.2(2), 29.4, $30.5\left(\mathrm{t},{ }^{3} \mathrm{~J}_{\mathrm{C}-\mathrm{F}} 2.9\right)$, $31.8,55.2,75.1\left(\mathrm{t},{ }^{2} J_{\mathrm{C}-\mathrm{F}} 29.9\right), 113.5,116.2\left(\mathrm{t},{ }^{3} J_{\mathrm{C}-\mathrm{F}} 8.8\right), 121.2(\mathrm{t}$, $\left.{ }^{1} J_{C-F} 247.2\right), 128.2\left(t,{ }^{3} J_{C-F} 2.2\right), 129.0,142.9\left(t,{ }^{2} J_{C-F} 22.6\right)$ and 159.8; $\delta_{\mathrm{F}}\left(471 \mathrm{MHz}, \mathrm{CDCl}_{3}\right)-109.52$ and -110.20 (both d, ${ }^{2} J_{\mathrm{F}-\mathrm{F}}$ 244.7); $m / z$ (GC/MS-Cl) $327\left(\mathrm{M}^{+}+1\right)$.

2,2-Difluoro-3-methylene-1-(3-chlorophenyl)undecan-1-ol 57c. $(60 \mathrm{mg}, 0.17 \mathrm{mmol}, 93 \%), R_{\mathrm{f}}=0.2$ (10:90 ether:light petroleum) (Found: $\mathrm{M}^{+}+\mathrm{NH}_{4}, 348.1897 . \mathrm{C}_{18} \mathrm{H}_{29} \mathrm{NO}^{35} \mathrm{ClF}_{2}$ requires $\mathrm{M}, 348.1900) ; v_{\max } / \mathrm{cm}^{-1} 3428,2954,2926,2855,1599,1576$, $1467,1433,1199,1163,1079,925$ and $776 ; \delta_{\mathrm{H}}\left(400 \mathrm{MHz}_{2} \mathrm{CDCl}_{3}\right)$ $0.90\left(3 \mathrm{H}, \mathrm{t}, \mathrm{J} 6.8,11-\mathrm{H}_{3}\right), 1.22-1.36\left(10 \mathrm{H}, \mathrm{m}, 5 \times \mathrm{CH}_{2}\right), 1.44(2 \mathrm{H}$, $\left.\mathrm{m}, 5-\mathrm{H}_{2}\right), 1.96\left(2 \mathrm{H}, \mathrm{t}, J\right.$ 8.0, 4- $\left.\mathrm{H}_{2}\right), 2.56(1 \mathrm{H}$, br. s, OH), $4.90(1 \mathrm{H}$, $\mathrm{t},{ }^{3} J_{\mathrm{H}-\mathrm{F}} 10.8,1-\mathrm{H}$ ), 5.22 and 5.32 (each $\left.1 \mathrm{H}, \mathrm{s}, 3-\mathrm{CH}\right), 7.26-7.35(3$ $\mathrm{H}, \mathrm{m}, \mathrm{ArH})$ and 7.42-7.44 $(1 \mathrm{H}, \mathrm{m}, \mathrm{ArH}) ; \delta_{\mathrm{C}}\left(100 \mathrm{MHz}, \mathrm{CDCl}_{3}\right)$ 14.1, 22.6, 27.6, 29.2, 29.4, $30.5\left(\mathrm{t},{ }^{3} J_{\mathrm{C}-\mathrm{F}} 2.9\right), 31.8,74.8\left(\mathrm{t},{ }^{2} J_{\mathrm{C}-\mathrm{F}}\right.$ 30.0), $116.7\left(t,{ }^{3} J_{C-F} 8.1\right), 120.9\left(t,{ }^{1} J_{C-F} 250.4\right), 126.0,128.0$, $128.8,129.3,134.1,138.0\left(\mathrm{t},{ }^{3} J_{\mathrm{C}-\mathrm{F}} 1.5\right)$ and $142.4\left(\mathrm{t},{ }^{2} J_{\mathrm{C}-\mathrm{F}} 22.8\right) ; \delta_{\mathrm{F}}$ (471 MHz, $\mathrm{CDCl}_{3}$ ) -109.99 and -108.92 (both d, ${ }^{2} J_{\mathrm{F}-\mathrm{F}} 244.7$ ); $\mathrm{m} / \mathrm{z}$ (APCI) $348.2\left(\mathrm{M}^{+}+18,100 \%\right)$.

5,5-Difluoro-6-methylenetetradecan-4-ol 57d. (50 mg, 0.18 $\mathrm{mmol}, 98 \%), R_{\mathrm{f}}=0.23$ (10:90 ether:light petroleum) (Found: $\mathrm{M}^{+}$ $+\mathrm{NH}_{4}, 280.2442$. $\mathrm{C}_{15} \mathrm{H}_{32} \mathrm{NOF}_{2}$ requires $\left.\mathrm{M}, 280.2446\right) ; v_{\max } / \mathrm{cm}^{-1}$ 3387, 2959, 2926, 2856, 1466, 1185, 1075, 1029, 964 and 922; $\delta_{\mathrm{H}}\left(400 \mathrm{MHz}, \mathrm{CDCl}_{3}\right) 0.89\left(3 \mathrm{H}, \mathrm{t}, J\right.$ J 6.6, 14- $\left.\mathrm{H}_{3}\right), 0.96(3 \mathrm{H}, \mathrm{t}, \mathrm{J}$ 7.0, 1- $\left.\mathrm{H}_{3}\right), 1.21-1.38\left(10 \mathrm{H}, \mathrm{m}, 5 \times \mathrm{CH}_{2}\right), 1.38-1.70\left(6 \mathrm{H}, \mathrm{m}, 3 \times \mathrm{CH}_{2}\right)$, $1.85(1 \mathrm{H}$, br. $\mathrm{s}, \mathrm{OH}), 2.04-2.19\left(2 \mathrm{H}, \mathrm{m}, 7-\mathrm{H}_{2}\right), 3.83(1 \mathrm{H}, \mathrm{q}, \mathrm{J} 9.6$, $4-\mathrm{H})$ and 5.23 and 5.44 (each $1 \mathrm{H}, \mathrm{s}, 6-\mathrm{CH}) ; \delta_{\mathrm{C}}\left(100 \mathrm{MHz} \mathrm{CDCl}_{3}\right)$ $13.8,14.1,18.8,22.6,27.8,29.2,29.3,29.4,30.5\left(t,{ }^{4} J_{C-F} 2.9\right)$, $31.8,31.9\left(\mathrm{t},{ }^{3} J_{C-F} 2.2\right), 72.3\left(t,{ }^{2} J_{C-F} 28.7\right), 115.5\left(t,{ }^{3} J_{C-F} 8.8\right), 121.8$ $\left(\mathrm{t},{ }^{1} J_{\mathrm{C}-\mathrm{F}} 247.5\right)$ and $142.9\left(\mathrm{t},{ }^{2} J_{\mathrm{C}-\mathrm{F}} 22.8\right) ; \delta_{\mathrm{F}}\left(470 \mathrm{MHz}, \mathrm{CDCl}_{3}\right)$ -112.29 and -111.60 (both $\left.d,{ }^{2} J_{F-F} 246.3\right) ; m / z$ (APCI) $280.2\left(\mathrm{M}^{+}+\right.$ $18,100 \%)$.

4,4-Difluoro-2-methyl-5-methylenetridecan-3-ol 57e. (33 mg, $0.126 \mathrm{mmol}, 68 \%), R_{\mathrm{f}}=0.39$ (10:90 ether:light petroleum) (Found: $\mathrm{M}^{+}+\mathrm{NH}_{4}, 280.2443 . \mathrm{C}_{15} \mathrm{H}_{32} \mathrm{NOF}_{2}$ requires $\mathrm{M}, 280.2446$ ); $v_{\max } / \mathrm{cm}^{-1} 3458,2958,2926,2856,1467,1264,1186,1077,1021$, 907 and $725 ; \delta_{\mathrm{H}}\left(400 \mathrm{MHz}, \mathrm{CDCl}_{3}\right) 0.89\left(3 \mathrm{H}, \mathrm{t}, J 6.8,13-\mathrm{H}_{3}\right), 0.99$ and 1.05 (each $3 \mathrm{H}, \mathrm{d}, J \mathrm{~J} .9,1-\mathrm{H}_{3}$ or $\left.2-\mathrm{CH}_{3}\right), 1.23-1.38(10 \mathrm{H}, \mathrm{m}, 5$ $\left.\times \mathrm{CH}_{2}\right), 1.51\left(2 \mathrm{H}, \mathrm{m}, 7-\mathrm{H}_{2}\right), 1.82(1 \mathrm{H}, \mathrm{br} . \mathrm{s}, \mathrm{OH}), 1.98(1 \mathrm{H}, \mathrm{m}, 2-$ $\mathrm{H}), 2.05-2.19\left(2 \mathrm{H}, \mathrm{m}, 6-\mathrm{H}_{2}\right), 3.66(1 \mathrm{H}, \mathrm{m}, 3-\mathrm{H})$ and 5.22 and 5.47 (each $1 \mathrm{H}, \mathrm{s}, 5-\mathrm{H}) ; \delta_{\mathrm{C}}\left(100 \mathrm{MHz}, \mathrm{CDCl}_{3}\right) 14.1,16.2\left(\mathrm{t},{ }^{4} J_{\mathrm{C}-\mathrm{F}} 2.2\right)$, $20.8\left(t,{ }^{4} J_{C-F} 1.5\right), 22.6,27.8,28.3,29.2,29.3,29.4,30.4\left(t,{ }^{3} J_{C-F}\right.$ 3.0), 31.8, $76.2\left(t,{ }^{2} J_{C-F} 27.3\right), 115.1\left(t,{ }^{3} J_{C-F} 9.6\right), 122.3\left(t,{ }^{1} J_{C-F}\right.$ 
248.2) and $143.4\left(t,{ }^{2} J_{C-F} 22.8\right) ; \delta_{F}\left(471 \mathrm{MHz}, \mathrm{CDCl}_{3}\right)-109.36$ and -108.49 (both d, ${ }^{2} J_{\mathrm{F}-\mathrm{F}} 248.2$ ); $\mathrm{m} / \mathrm{z}$ (APCI) $280.2\left(\mathrm{M}^{+}+18,100 \%\right)$.

4,4-Difluoro-2,2-dimethyl-5-methylenetridecan-3-ol 57f. (24 $\mathrm{mg}, 0.087 \mathrm{mmol}, 47 \%), R_{\mathrm{f}}=0.29$ (10:90 ether:light petroleum) (Found: $\mathrm{M}^{+}+\mathrm{NH}_{4}, 294.2602 . \mathrm{C}_{16} \mathrm{H}_{34} \mathrm{NOF}_{2}$ requires $\mathrm{M}, 294.2603$ ); $v_{\text {max }} / \mathrm{cm}^{-1} 3491,2957,2925,2856,1741,1714,1466,1368,1166$, 1057,1016 and 922; $\delta_{\mathrm{H}}\left(500 \mathrm{MHz}, \mathrm{CDCl}_{3}\right)$ 0.84-0.92 (3 H, m, 13$\left.\mathrm{H}_{3}\right), 1.07\left[9 \mathrm{H}, \mathrm{s}, \mathrm{C}\left(\mathrm{CH}_{3}\right)_{3}\right], 1.23-1.38\left(10 \mathrm{H}, \mathrm{m}, 5 \times \mathrm{CH}_{2}\right), 1.52(2 \mathrm{H}$, m, 7- $\left.\mathrm{H}_{2}\right), 1.83\left(1 \mathrm{H}\right.$, br. s, OH), $2.15\left(2 \mathrm{H}, \mathrm{t}, \mathrm{J} 7.9,6-\mathrm{H}_{2}\right), 3.52(1 \mathrm{H}$, $\left.\mathrm{dd},{ }^{3} J_{\mathrm{H}-\mathrm{F}} 19.9,6.3,3-\mathrm{H}\right), 5.21\left(1 \mathrm{H}, \mathrm{d},{ }^{4} J_{\mathrm{H}-\mathrm{F}} 1.6,5-\mathrm{CH}\right)$ and $5.47(1 \mathrm{H}$, d, $\left.{ }^{4} J_{\mathrm{H}-\mathrm{F}} 2.5,5-\mathrm{H}^{\prime}\right) ; \delta_{\mathrm{C}}\left(100 \mathrm{MHz}, \mathrm{CDCl}_{3}\right) 14.1,22.7,26.9\left(\mathrm{t},{ }^{4} J_{\mathrm{C}-\mathrm{F}}\right.$ 2.2), 27.8, 29.2, 29.4(2), 30.5 (dd, $\left.{ }^{3} J_{\mathrm{C}-\mathrm{F}} 3.7,2.2\right), 31.9,34.8,78.1$ (dd, $\left.{ }^{2} J_{\text {C-F }} 29.5,26.5\right), 114.8\left(d d,{ }^{3} J_{C-F} 10.3,8.1\right), 123.0\left(d d,{ }^{1} J_{C-F}\right.$ $253.4,249.0)$ and $144.7\left(\mathrm{t},{ }^{2} \mathrm{~J}_{\mathrm{C}-\mathrm{F}} 22.8\right) ; \delta_{\mathrm{F}}\left(471 \mathrm{MHz}, \mathrm{CDCl}_{3}\right)$ -111.59 and -100.68 (both $d,{ }^{2} J_{F-F} 249.9$ ); $m / z\left(E^{+}\right) 277.3\left(\mathrm{M}^{+}+\right.$ $1,20 \%)$.

1-tert-Butyldiphenylsilyloxy-4,4-difluoro-5-

methylenetridecan-3-ol 57g. (85 mg, $0.169 \mathrm{mmol}, 91 \%), R_{\mathrm{f}}=0.27$ (10:90 ether:light petroleum) (Found: $\mathrm{M}^{+}+\mathrm{H}, \quad 503.3138$. $\mathrm{C}_{30} \mathrm{H}_{45} \mathrm{O}_{2} \mathrm{~F}_{2} \mathrm{Si}$ requires $\left.\mathrm{M}, 503.3151\right) ; v_{\text {max }} / \mathrm{cm}^{-1} 3471,2956,2928$, $2856,1427,1106,1084,936,822$ and $737 ; \delta_{\mathrm{H}}\left(400 \mathrm{MHz}_{1} \mathrm{CDCl}_{3}\right)$ $0.90\left(3 \mathrm{H}, \mathrm{t}, \mathrm{J}\right.$ 8.6, $\left.13-\mathrm{H}_{3}\right), 1.06\left[9 \mathrm{H}, \mathrm{s}, \mathrm{SiC}\left(\mathrm{CH}_{3}\right)_{3}\right], 1.24-1.39(10 \mathrm{H}$, $\left.\mathrm{m}, 5 \times \mathrm{CH}_{2}\right), 1.53\left(2 \mathrm{H}, \mathrm{m}, 7-\mathrm{H}_{2}\right), 1.81$ and 1.88 (each $\left.1 \mathrm{H}, \mathrm{m}, 2-\mathrm{H}\right)$, 2.08-2.23 $\left(2 \mathrm{H}, \mathrm{m}, 6-\mathrm{H}_{2}\right), 3.09(1 \mathrm{H}, \mathrm{br} . \mathrm{s}, \mathrm{OH}), 3.86$ and 3.96 (each $1 \mathrm{H}, \mathrm{m}, 1-\mathrm{H}), 4.20(1 \mathrm{H}, \mathrm{m}, 3-\mathrm{H}), 5.25$ and 5.48 (each $1 \mathrm{H}, \mathrm{s}$, 5-CH), 7.37-7.49 (6 H, m, ArH) and 7.63-7.73 (4 H, m, ArH); $\delta_{c}$ (125 MHz, $\mathrm{CDCl}_{3}$ ) 14.1, 19.1, 22.7, 26.8, 27.8, 29.3, 29.4(2), 30.5, $31.7,31.9,61.9,71.3\left(\mathrm{t},{ }^{2} J_{\mathrm{C}-\mathrm{F}} 30.7\right), 115.4\left(\mathrm{t},{ }^{3} J_{\mathrm{C}-\mathrm{F}} 9.1\right), 121.4(\mathrm{dd}$, $\left.{ }^{1} J_{C-F} 248.4,245.7\right), 127.8,129.9,132.9,133.0,135.5(2)$ and 143.0 $\left(\mathrm{t},{ }^{2} J_{\mathrm{C}-\mathrm{F}} 22.7\right) ; \delta_{\mathrm{F}}\left(377 \mathrm{MHz}, \mathrm{CDCl}_{3}\right)-114.15$ and -110.23 (both $\mathrm{d}$, $\left.{ }^{2} J_{F-F} 247.3\right) ; m / z(A P C l) 503.3\left(M^{+}+1,100 \%\right)$.

\section{1-tert-Butyldiphenylsilyloxy-4,4-difluorohex-5-en-3-one} (58). N-Methylmorpholine- $N$-oxide $(52 \mathrm{mg}, 0.446 \mathrm{mmol}$ ) and TPAP ( $5 \mathrm{mg}, 0.015 \mathrm{mmol}$ ) were added to the alcohol 40 (0.116 g, $0.297 \mathrm{mmol})$ and $4 \AA \AA$ molecular sieves $(0.168 \mathrm{~g})$ in dry DCM $(2.0$ $\mathrm{mL}$ ) and the mixture stirred for $16 \mathrm{~h}$ then concentrated under reduced pressure. Chromatography of the residue (light petroleum to $2: 98$ ether:light petroleum) gave the title compound 58 (90 mg, $0.23 \mathrm{mmol}, 78 \%$ ) as a colourless oil, $R_{\mathrm{f}}=$ 0.43 (10:90 ether:light petroleum) (Found: $\mathrm{M}^{+}+\mathrm{Na}, 411.1568$. $\mathrm{C}_{22} \mathrm{H}_{26} \mathrm{O}_{2} \mathrm{~F}_{2} \mathrm{SiNa}$ requires $\left.\mathrm{M}, 411.1568\right) ; v_{\max } / \mathrm{cm}^{-1} 2957,2931$, 2857, 1746, 1427, 1107, 998, 956, 821 and 738; $\delta_{\mathrm{H}}(500 \mathrm{MHz}$, $\left.\mathrm{CDCl}_{3}\right) 1.05\left[9 \mathrm{H}, \mathrm{s}, \mathrm{SiC}\left(\mathrm{CH}_{3}\right)_{3}\right], 2.92\left(2 \mathrm{H}, \mathrm{t}, J 6.2,2-\mathrm{H}_{2}\right), 4.00(2 \mathrm{H}$, $\mathrm{t}, J$ 6.2, 1- $\left.\mathrm{H}_{2}\right), 5.66(1 \mathrm{H}, \mathrm{d}, J$ J 11.0, 6- H), $5.85(1 \mathrm{H}, \mathrm{dt}, J$ 17.4, 2.5, 6- $\left.\mathrm{H}^{\prime}\right), 6.01(1 \mathrm{H}, \mathrm{m}, 5-\mathrm{H}), 7.39-7.48(6 \mathrm{H}, \mathrm{m}, \mathrm{ArH})$ and 7.67-7.71 $(4 \mathrm{H}, \mathrm{m}, \mathrm{ArH}) ; \delta_{\mathrm{C}}\left(100 \mathrm{MHz}, \mathrm{CDCl}_{3}\right)$ 19.1, 26.7, 39.4, 58.2, 114.3 $\left(t,{ }^{1} J_{C-F} 251.2\right), 123.1\left(t,{ }^{3} J_{C-F} 9.6\right), 127.7,128.3\left(t,{ }^{2} J_{C-F} 25.1\right)$, $129.8,133.2,135.5$ and $198.2\left(t,{ }^{2} J_{C-F} 32.4\right) ; \delta_{F}\left(471 \mathrm{MHz} \mathrm{CDCl}_{3}\right)$ $-108.32 ; \mathrm{m} / z\left(\mathrm{ES}^{+}\right) 427\left(\mathrm{M}^{+}+39,25 \%\right), 411\left(\mathrm{M}^{+}+23,15\right)$ and $102(100)$.

Methyl (E)- and (Z)-3-(2-tert-butyldiphenylsilyloxyethyl)4,4-difluorohexa-2,5-dienoate (59) and (60). Methoxycarbonylmethylene(triphenyl)phosphorane $(0.28 \mathrm{~g}$, $0.844 \mathrm{mmol}$ ) was added to the ketone $58(0.27 \mathrm{~g}, 0.703 \mathrm{mmol})$ in dry toluene $(2.5 \mathrm{~mL})$ and the mixture heated at $70^{\circ} \mathrm{C}$ for $6 \mathrm{~h}$.
After concentration under reduced pressure, chromatography of the residue (light petroleum to $0.5: 99.5$ ether:light petroleum) gave the title compound 59 ( $0.26 \mathrm{~g}, 0.59 \mathrm{mmol}, 84 \%)$ as a colourless oil, $R_{f}=0.38$ (10:90 ether:light petroleum) (Found: $\mathrm{M}^{+}+\mathrm{Na}, 467.1841 . \mathrm{C}_{25} \mathrm{H}_{30} \mathrm{O}_{3} \mathrm{~F}_{2} \mathrm{SiNa}$ requires $\mathrm{M}$, 467.1824); $v_{\max } / \mathrm{cm}^{-1} 2953,2931,2857,1728,1428,1258,1199$, $1178,1106,1089,981,822$ and $739 ; \delta_{\mathrm{H}}\left(400 \mathrm{MHz}, \mathrm{CDCl}_{3}\right) 1.05[9$ $\left.\mathrm{H}, \mathrm{s}, \mathrm{SiC}\left(\mathrm{CH}_{3}\right)_{3}\right], 2.99\left(2 \mathrm{H}, \mathrm{t}, J 6.8,1^{\prime}-\mathrm{H}_{2}\right), 3.70\left(3 \mathrm{H}, \mathrm{s}, \mathrm{OCH}_{3}\right), 3.81$ $\left(2 \mathrm{H}, \mathrm{t}, \mathrm{J} 6.8,2^{\prime}-\mathrm{H}_{2}\right), 5.45(1 \mathrm{H}, \mathrm{d}, J \mathrm{~J} 10.8,6-\mathrm{H}), 5.63(1 \mathrm{H}, \mathrm{dt}, \mathrm{J} 17.4$, 2.2, 6- $\left.\mathrm{H}^{\prime}\right), 5.76-5.90(1 \mathrm{H}, \mathrm{m}, 5-\mathrm{H}), 6.21(1 \mathrm{H}, \mathrm{s}, 2-\mathrm{H}), 7.37-7.46(6$ $\mathrm{H}, \mathrm{m}, \mathrm{ArH})$ and 7.66-7.71 $(4 \mathrm{H}, \mathrm{m}, \mathrm{ArH}) ; \delta_{\mathrm{C}}\left(100 \mathrm{MHz}, \mathrm{CDCl}_{3}\right)$ 19.1, 26.8, $30.5\left(\mathrm{t},{ }^{3} J_{\mathrm{C}-\mathrm{F}} 1.5\right), 51.5,62.4,118.7\left(\mathrm{t},{ }^{1} J_{\mathrm{C}-\mathrm{F}} 243.1\right)$, $120.9\left(\mathrm{t},{ }^{3} J_{\mathrm{C}-\mathrm{F}} 9.6\right), 121.2\left(\mathrm{t},{ }^{3} J_{\mathrm{C}-\mathrm{F}} 8.8\right), 127.6,129.6,131.4\left(\mathrm{t},{ }^{2} J_{\mathrm{C}-\mathrm{F}}\right.$ 28.7), 133.7, 135.6, $149.4\left(\mathrm{t},{ }^{2} J_{\mathrm{C}-\mathrm{F}} 25.0\right)$ and $165.6 ; \delta_{\mathrm{F}}(471 \mathrm{MHz}$, $\left.\mathrm{CDCl}_{3}\right)-100.52 ; \mathrm{m} / z\left(\mathrm{ES}^{+}\right) 467.3\left(\mathrm{M}^{+}+23,100 \%\right)$. The second fraction was the title compound 60 ( $36 \mathrm{mg}, 0.081 \mathrm{mmol}, 11 \%$ ) as a colourless oil, $R_{\mathrm{f}}=0.27$ (10:90 ether:light petroleum) (Found: $\mathrm{M}^{+}+\mathrm{Na}, 467.1836 . \mathrm{C}_{25} \mathrm{H}_{30} \mathrm{O}_{3} \mathrm{~F}_{2} \mathrm{SiNa}$ requires $\left.\mathrm{M}, 467.1824\right)$; $v_{\max } / \mathrm{cm}^{-1} 2953,2931,2857,1735,1428,1240,1196,1159,1105$, 997, 822 and 737; $\delta_{\mathrm{H}}\left(400 \mathrm{MHz}, \mathrm{CDCl}_{3}\right) 1.06\left[9 \mathrm{H}, \mathrm{s}, \mathrm{SiC}\left(\mathrm{CH}_{3}\right)_{3}\right]$, $2.41\left(2 \mathrm{H}, \mathrm{td}, J 6.4,0.8,1^{\prime}-\mathrm{H}_{2}\right), 3.74\left(3 \mathrm{H}, \mathrm{s}, \mathrm{OCH}_{3}\right), 3.80(2 \mathrm{H}, \mathrm{t}, J$ $\left.6.4,2^{\prime}-H_{2}\right), 5.43(1 \mathrm{H}, \mathrm{d}, J 11.3,6-\mathrm{H}), 5.63(1 \mathrm{H}, \mathrm{dt}, J 17.3,2.8,6-$ $\left.\mathrm{H}^{\prime}\right), 5.98(1 \mathrm{H}, \mathrm{s}, 2-\mathrm{H}), 6.16(1 \mathrm{H}, \mathrm{m}, 5-\mathrm{H}), 7.36-7.47(6 \mathrm{H}, \mathrm{m}, \mathrm{ArH})$ and 7.63-7.69 $(4 \mathrm{H}, \mathrm{m}, \mathrm{ArH}) ; \delta_{\mathrm{C}}\left(100 \mathrm{MHz}, \mathrm{CDCl}_{3}\right)$ 19.1, 26.8, 34.6 $\left(t,{ }^{3} J_{C-F} 3.7\right), 51.8,61.8,118.7\left(t,{ }^{1} J_{C-F} 239.9\right), 120.1\left(t,{ }^{3} J_{C-F} 8.8\right)$, $122.9\left(t,{ }^{3} J_{C-F} 5.1\right), 127.7,129.7,132.0\left(t,{ }^{2} J_{C-F} 27.8\right), 133.4,135.6$, $142.8\left(\mathrm{t},{ }^{2} \mathrm{~J}_{\mathrm{C}-\mathrm{F}} 27.0\right)$ and $166.2 ; \delta_{\mathrm{F}}\left(377 \mathrm{MHz}, \mathrm{CDCl}_{3}\right)-95.73 ; \mathrm{m} / \mathrm{z}$ $\left(\mathrm{ES}^{+}\right) 467.3\left(\mathrm{M}^{+}+23,100 \%\right)$ and $462.4\left(\mathrm{M}^{+}+18,85\right)$.

\section{1-tert-Butyldiphenylsilyloxy-4,4-difluoro-5-}

methylenetridecan-3-one (61). Anhydrous DMSO (51 $\mu \mathrm{L}, 0.723$ $\mathrm{mmol}$ ) was added dropwise to oxalyl chloride (31 $\mu \mathrm{L}, 0.362$ $\mathrm{mmol})$ in dry DCM $(1.6 \mathrm{~mL})$ at $-78{ }^{\circ} \mathrm{C}$. After $30 \mathrm{~min}$, the alcohol $57 \mathrm{~g}(91 \mathrm{mg}, 0.18 \mathrm{mmol})$ in dry DCM $(1.0 \mathrm{~mL})$ was added. After a further $45 \mathrm{~min}$, triethylamine $(0.20 \mathrm{~mL}, 1.45 \mathrm{mmol})$ was added and the mixture was stirred at rt for $1 \mathrm{~h}$. Saturated aqueous ammonium chloride $(10 \mathrm{~mL})$ and $\mathrm{DCM}(10 \mathrm{~mL})$ were added and the aqueous phase was extracted into DCM $(3 \times 10 \mathrm{~mL})$. The organic extracts were washed with brine $(40 \mathrm{~mL})$, dried $\left(\mathrm{MgSO}_{4}\right)$ and concentrated under reduced pressure. Chromatography of the residue (0.25:99.75 ether:light petroleum) gave the title compound 61 (55 mg, $0.11 \mathrm{mmol}, 61 \%)$ as a colourless oil, $R_{\mathrm{f}}=$ 0.53 (5:95 ether:light petroleum) (Found: $\mathrm{M}^{+}+\mathrm{Na}, 523.2809$. $\mathrm{C}_{30} \mathrm{H}_{42} \mathrm{O}_{2} \mathrm{~F}_{2} \mathrm{SiNa}$ requires $\left.\mathrm{M}, 523.2820\right) ; v_{\max } / \mathrm{cm}^{-1} 2955,2928$, $2857,1749,1428,1106,927,822$ and $737 ; \delta_{\mathrm{H}}\left(500 \mathrm{MHz}_{2} \mathrm{CDCl}_{3}\right)$ $0.89\left(3 \mathrm{H}, \mathrm{t}, \mathrm{J}\right.$ 6.7, 13- $\left.\mathrm{H}_{3}\right), 1.03\left[9 \mathrm{H}, \mathrm{s}, \mathrm{SiC}\left(\mathrm{CH}_{3}\right)_{3}\right], 1.22-1.34(10 \mathrm{H}$, $\left.\mathrm{m}, 5 \times \mathrm{CH}_{2}\right), 1.47\left(2 \mathrm{H}, \mathrm{m}, 7-\mathrm{H}_{2}\right), 2.08\left(2 \mathrm{H}, \mathrm{t}, \mathrm{J}\right.$ 7.6, 6- $\left.\mathrm{H}_{2}\right), 2.88(2$ $\left.\mathrm{H}, \mathrm{t}, J 6.0,2-\mathrm{H}_{2}\right), 3.97\left(2 \mathrm{H}, \mathrm{t}, J 6.0,1-\mathrm{H}_{2}\right), 5.32$ and 5.55 (each 1 $\mathrm{H}, \mathrm{s}, 5-\mathrm{CH}), 7.37-7.47(6 \mathrm{H}, \mathrm{m}, \mathrm{ArH})$ and 7.65-7.70 (4 H, m, ArH); $\delta_{\mathrm{C}}\left(100 \mathrm{MHz}, \mathrm{CDCl}_{3}\right) 14.1,19.1,22.6,26.7,27.5,29.2,29.3,29.4$ $\left(\mathrm{t},{ }^{4} J_{\mathrm{C}-\mathrm{F}} 2.2\right), 31.8,39.3,58.0,116.1\left(\mathrm{t},{ }^{1} J_{\mathrm{C}-\mathrm{F}} 251.6\right), 117.1\left(\mathrm{t},{ }^{3} J_{\mathrm{C}-\mathrm{F}}\right.$ 8.8), 127.7, 129.7, 133.2, 135.5, $140.8\left(\mathrm{t},{ }^{2} \mathrm{~J}_{\mathrm{C}-\mathrm{F}} 21.9\right)$ and $198.3(\mathrm{t}$, $\left.{ }^{2} \mathrm{~J}_{\mathrm{C}-\mathrm{F}} 31.4\right) ; \delta_{\mathrm{F}}\left(471 \mathrm{MHz}, \mathrm{CDCl}_{3}\right)-109.74 ; \mathrm{m} / \mathrm{z}\left(\mathrm{ES}^{+}\right) 523.4\left(\mathrm{M}^{+}+\right.$ $23,100 \%)$.

Methyl (E)-3-(2-tert-butyldiphenylsilyloxyethyl)-4,4difluoro-5-methylenetridec-2-enoate (62). Methoxycarbonyl- 
methylidene(triphenyl)phosphorane $(37 \mathrm{mg}, 0.11 \mathrm{mmol}$ ) was added to the ketone $61(32 \mathrm{mg}, 0.064 \mathrm{mmol})$ in toluene $(0.23$ $\mathrm{mL}$ ) and the mixture heated at $70{ }^{\circ} \mathrm{C}$ for $11 \mathrm{~h}$. After concentration under reduced pressure, chromatography of the residue (0.25:99.75 ether:light petroleum) gave the title compound 62 (17 mg, $0.031 \mathrm{mmol}, 48 \%)$ as a colourless oil, $R_{\mathrm{f}}=$ 0.23 (5:95 ether:light petroleum) (Found: $\mathrm{M}^{+}+\mathrm{Na}, 579.3080$. $\mathrm{C}_{33} \mathrm{H}_{46} \mathrm{O}_{3} \mathrm{~F}_{2} \mathrm{SiNa}$ requires $\left.\mathrm{M}, 579.3082\right) ; v_{\max } / \mathrm{cm}^{-1} 2928,2856$, $1729,1428,1256,1200,1177,1105$ and $739 ; \delta_{\mathrm{H}}(500 \mathrm{MHz}$, $\left.\mathrm{CDCl}_{3}\right) 0.90\left(3 \mathrm{H}, \mathrm{t}, J 6.8,13-\mathrm{H}_{3}\right), 1.04\left[9 \mathrm{H}, \mathrm{s}, \mathrm{SiC}\left(\mathrm{CH}_{3}\right)_{3}\right], 1.21-$ $1.35\left(10 \mathrm{H}, \mathrm{m}, 5 \times \mathrm{CH}_{2}\right), 1.42\left(2 \mathrm{H}, \mathrm{m}, 7-\mathrm{H}_{2}\right), 1.94(2 \mathrm{H}, \mathrm{t}, \mathrm{J}$ 7.3, 6$\left.\mathrm{H}_{2}\right), 2.94\left(2 \mathrm{H}, \mathrm{t}, J \mathrm{~J} .7,1^{\prime}-\mathrm{H}_{2}\right), 3.70\left(3 \mathrm{H}, \mathrm{s}, \mathrm{OCH}_{3}\right), 3.76(2 \mathrm{H}, \mathrm{t}, J$ 7.7, 2' $\left.-\mathrm{H}_{2}\right), 5.17$ and 5.40 (each $\left.1 \mathrm{H}, \mathrm{s}, 5-\mathrm{CH}\right), 6.22(1 \mathrm{H}, \mathrm{t}, \mathrm{J}$ 1.8, 2$\mathrm{H})$, 7.35-7.47 (6 H, m, ArH) and 7.66-7.72 $(4 \mathrm{H}, \mathrm{m}, \mathrm{ArH}) ; \delta_{\mathrm{c}}(125$ $\left.\mathrm{MHz}, \mathrm{CDCl}_{3}\right)$ 14.1, 19.1, 22.6, 26.7, 27.5, 29.2(2), 29.4, 29.5, 30.7, $31.8,51.5,62.4,115.9\left(\mathrm{t},{ }^{3} J_{\mathrm{C}-\mathrm{F}} 8.1\right), 120.6\left(\mathrm{t},{ }^{1} J_{\mathrm{C}-\mathrm{F}} 243.7\right), 121.3(\mathrm{t}$, $\left.{ }^{3} J_{C-F} 9.0\right), 127.6,129.5,133.7,135.6,142.5\left(t,{ }^{2} J_{C-F} 24.4\right), 148.9(t$, $\left.{ }^{2} J_{\mathrm{C}-\mathrm{F}} 25.3\right)$ and $165.6 ; \delta_{\mathrm{F}}\left(377 \mathrm{MHz}, \mathrm{CDCl}_{3}\right)-101.98 ; \mathrm{m} / \mathrm{z}\left(\mathrm{ES}^{+}\right)$ $579.4\left(\mathrm{M}^{+}+23,100 \%\right)$.

Ethyl 5-tert-butyldiphenylsilyloxy-2,2-difluoro-3oxopentanoate (63). With rigorous exclusion of $\mathrm{O}_{2}, \mathrm{Sml}_{2}(0.1 \mathrm{M}$ in THF, $48 \mathrm{~mL}, 48 \mathrm{mmol}$ ) was added to the aldehyde $39(0.50 \mathrm{~g}$, $1.6 \mathrm{mmol}$ ) and ethyl 2-bromo-2,2-difluoroacetate $11(0.22 \mathrm{~mL}$, $1.76 \mathrm{mmol})$ in dry THF $(16 \mathrm{~mL})$ and the yellow mixture was stirred at $\mathrm{rt}$ for $10 \mathrm{~min}$. Aqueous hydrogen chloride $(1 \mathrm{M}, 50 \mathrm{~mL}$ ) was added slowly followed by ether $(50 \mathrm{~mL})$. The aqueous phase was extracted with ether $(3 \times 50 \mathrm{~mL})$ and the organic extracts were washed with saturated aqueous sodium thiosulfate (50 $\mathrm{mL})$ then brine $(250 \mathrm{~mL})$ and dried $\left(\mathrm{MgSO}_{4}\right)$. After concentration under reduced pressure, chromatography of the residue (0.5:99.5 to 10:90 ether:light petroleum) gave ethyl 5-tertbutyldiphenylsilyloxy-2,2-difluoro-3-hydroxypentanoate $(0.68 \mathrm{~g}$, $1.57 \mathrm{mmol}, 98 \%)$ as a colourless oil, $R_{\mathrm{f}}=0.09$ (10:90 ether:light petroleum) (Found: $\mathrm{M}^{+}+\mathrm{Na}, 459.1797 . \mathrm{C}_{23} \mathrm{H}_{30} \mathrm{O}_{4} \mathrm{~F}_{2} \mathrm{SiNa}$ requires $\mathrm{M}, 459.1779) ; v_{\max } / \mathrm{cm}^{-1} 3476,3071,2959,2931,2888,2858$, $1759,1472,1446,1307,1206,1106,822$ and $738 ; \delta_{H}(400 \mathrm{MHz}$, $\left.\mathrm{CDCl}_{3}\right) 1.06\left[9 \mathrm{H}, \mathrm{s}, \mathrm{SiC}\left(\mathrm{CH}_{3}\right)_{3}\right], 1.39\left(3 \mathrm{H}, \mathrm{t}, J 7.0, \mathrm{OCH}_{2} \mathrm{CH}_{3}\right), 1.87-$ $1.95\left(2 \mathrm{H}, \mathrm{m}, 4-\mathrm{H}_{2}\right), 3.42(1 \mathrm{H}$, br. s, 3-OH), 3.85-4.01 $(2 \mathrm{H}, \mathrm{m}, 5-$ $\left.\mathrm{H}_{2}\right), 4.39\left(2 \mathrm{H}, \mathrm{q}, \mathrm{J} 7.0, \mathrm{OCH}_{2} \mathrm{CH}_{3}\right), 4.44(1 \mathrm{H}, \mathrm{m}, 3-\mathrm{H}), 7.35-7.50(6$ $\mathrm{H}, \mathrm{m}, \mathrm{ArH})$ and 7.61-7.75 (4 H, m, ArH); $\delta_{\mathrm{C}}\left(125 \mathrm{MHz}, \mathrm{CDCl}_{3}\right)$ 13.9, 19.0, 26.8, 30.8, 61.7, 62.9, 70.9 (dd, $\left.{ }^{2} J_{\text {C-F }} 29.4,24.8\right), 114.6$ (dd, $\left.{ }^{1} J_{C-F} 262.6,252.5\right), 127.8,129.9(2), 132.7,132.8,135.5(2)$ and $163.6\left(\mathrm{dd},{ }^{2} \mathrm{~J}_{\mathrm{C}-\mathrm{F}} 33.1,31.2\right) ; \delta_{\mathrm{F}}\left(377 \mathrm{MHz}, \mathrm{CDCl}_{3}\right)-123.62$ and -114.47 (both $\left.d,{ }^{2} J_{F-F} 261.2\right) ; m / z\left(E^{+}\right) 459.3\left(\mathrm{M}^{+}+23,90 \%\right)$ and 102 (100).

$\mathrm{N}$-Methylmorpholine- $\mathrm{N}$-oxide $(83 \mathrm{mg}, 0.71 \mathrm{mmol}$ ) and TPAP ( $8 \mathrm{mg}, 0.024 \mathrm{mmol}$ ) were added to a suspension of this alcohol $(0.21 \mathrm{~g}, 0.47 \mathrm{mmol})$ and $4 \AA \AA$ molecular sieves $(0.27 \mathrm{~g})$ in DCM (2.7 $\mathrm{mL}$ ) and the mixture stirred at $\mathrm{rt}$ for $16 \mathrm{~h}$. After concentration under reduced pressure, chromatography of the residue (light petroleum to $0.25: 99.75$ ether:light petroleum) gave the title compound $63(0.13 \mathrm{~g}, 0.30 \mathrm{mmol}, 63 \%)$ as a colourless oil, $R_{\mathrm{f}}=$ 0.29 (10:90 ether:light petroleum) (Found: $\mathrm{M}^{+}+\mathrm{Na}, 457.1619$. $\mathrm{C}_{23} \mathrm{H}_{28} \mathrm{O}_{4} \mathrm{~F}_{2} \mathrm{SiNa}$ requires $\left.\mathrm{M}, 457.1617\right) ; v_{\max } / \mathrm{cm}^{-1} 3071,2957$, 2931, 2889, 2857, 1777, 1751, 1428, 1111, 822 and 739; $\delta_{H}(500$ $\left.\mathrm{MHz}, \mathrm{CDCl}_{3}\right) 1.04\left[9 \mathrm{H}, \mathrm{s}, \mathrm{SiC}\left(\mathrm{CH}_{3}\right)_{3}\right], 1.35\left(3 \mathrm{H}, \mathrm{t}, \mathrm{J}\right.$ 7.2, $\left.\mathrm{OCH}_{2} \mathrm{CH}_{3}\right)$, $2.99\left(2 \mathrm{H}, \mathrm{t}, J 6.0,4-\mathrm{H}_{2}\right), 4.00\left(2 \mathrm{H}, \mathrm{t}, \mathrm{J} 6.0,5-\mathrm{H}_{2}\right), 4.37(2 \mathrm{H}, \mathrm{q}, J$ 7.2, $\left.\mathrm{OCH}_{2} \mathrm{CH}_{3}\right), 7.38-7.48(6 \mathrm{H}, \mathrm{m}, \mathrm{ArH})$ and 7.66-7.71 $(4 \mathrm{H}, \mathrm{m}$, $\mathrm{ArH}) ; \delta_{\mathrm{C}}\left(100 \mathrm{MHz}, \mathrm{CDCl}_{3}\right) 13.8,19.1,26.6,39.6,57.8,63.7$, $108.1\left(\mathrm{t},{ }^{1} J_{\mathrm{C}-\mathrm{F}} 262.5\right), 127.7,129.8,133.1,135.5,161.2\left(\mathrm{t},{ }^{2} J_{\mathrm{C}-\mathrm{F}}\right.$ 30.6) and $195.5\left(\mathrm{t},{ }^{2} \mathrm{~J}_{\mathrm{C}-\mathrm{F}} 28.4\right) ; \delta_{\mathrm{F}}\left(471 \mathrm{MHz}, \mathrm{CDCl}_{3}\right)-114.16 ; \mathrm{m} / \mathrm{z}$ $\left(\mathrm{ES}^{+}\right) 457.2\left(\mathrm{M}^{+}+23,60 \%\right)$.

Methyl (E)- and (Z)-5-tert-butyldiphenylsilyloxy-3(ethoxycarbonyldifluoromethyl)pent-2-enoate (64) and (65). Methoxycarbonylmethylidene(triphenyl)phosphorane $(0.12 \mathrm{~g}$, $0.36 \mathrm{mmol}$ ) was added to the ketone $63(0.13 \mathrm{~g}, 0.30 \mathrm{mmol})$ in toluene $(1.10 \mathrm{~mL})$ and the mixture stirred at $90{ }^{\circ} \mathrm{C}$ for $16 \mathrm{~h}$. After concentration under reduced pressure, chromatography of the residue (light petroleum to 2:98 ether:light petroleum) gave the title compound $64(0.12 \mathrm{~g}, 0.24 \mathrm{mmol}, 82 \%)$ as a colourless oil, $R_{\mathrm{f}}$ $=0.20$ (10:90 ether:light petroleum) (Found: $\mathrm{M}^{+}+\mathrm{Na}, 513.1893$. $\mathrm{C}_{26} \mathrm{H}_{32} \mathrm{O}_{5} \mathrm{~F}_{2} \mathrm{SiNa}$ requires $\left.\mathrm{M}, 513.1885\right) ; v_{\text {max }} / \mathrm{cm}^{-1} 2955,2932$, $2858,1769,1731,1279,1262,1201,1181,1109,1065,823$ and 740; $\delta_{\mathrm{H}}\left(400 \mathrm{MHz}, \mathrm{CDCl}_{3}\right) 1.04\left[9 \mathrm{H}, \mathrm{s}, \mathrm{SiC}\left(\mathrm{CH}_{3}\right)_{3}\right], 1.29(3 \mathrm{H}, \mathrm{t}, J$ 7.3, $\left.\mathrm{OCH}_{2} \mathrm{CH}_{3}\right), 3.06\left(2 \mathrm{H}, \mathrm{t}, J \mathrm{~J} .2,4-\mathrm{H}_{2}\right), 3.70\left(3 \mathrm{H}, \mathrm{s}, \mathrm{OCH}_{3}\right), 3.78$ $\left(2 \mathrm{H}, \mathrm{t}, J 7.2,5-\mathrm{H}_{2}\right), 4.26\left(2 \mathrm{H}, \mathrm{q}, J 7.3, \mathrm{OCH}_{2} \mathrm{CH}_{3}\right), 6.33(1 \mathrm{H}, \mathrm{s}, 2-$ $\mathrm{H})$, 7.36-7.46 $(6 \mathrm{H}, \mathrm{m}, \mathrm{ArH})$ and 7.66-7.70 $(4 \mathrm{H}, \mathrm{m}, \mathrm{ArH}) ; \delta_{\mathrm{C}}(100$ $\left.\mathrm{MHz}, \mathrm{CDCl}_{3}\right) 13.8,19.1,26.7,30.1$ (t, $\left.{ }^{3} J_{\mathrm{C}-\mathrm{F}} 1.5\right), 51.6,62.0,63.4$, $113.1\left(\mathrm{t},{ }^{1} J_{\mathrm{C}-\mathrm{F}} 254.9\right), 123.3\left(\mathrm{t},{ }^{3} \mathrm{~J}_{\mathrm{C}-\mathrm{F}} 8.8\right), 127.6,129.6,133.5$, 135.5, $146.0\left(\mathrm{t},{ }^{2} \mathrm{~J}_{\mathrm{C}-\mathrm{F}} 22.1\right), 162.9\left(\mathrm{t},{ }^{2} \mathrm{~J}_{\mathrm{C}-\mathrm{F}} 33.9\right)$ and $165.0 ; \delta_{\mathrm{F}}(377$ $\left.\mathrm{MHz}, \mathrm{CDCl}_{3}\right)-107.08 ; \mathrm{m} / \mathrm{z}\left(\mathrm{ES}^{+}\right) 513.2\left(\mathrm{M}^{+}+23,80 \%\right), 491.2\left(\mathrm{M}^{+}\right.$ $+1,20)$ and $256.9(100)$. The second fraction was the title compound 65 (17 mg, $0.035 \mathrm{mmol}, 12 \%)$ as a colourless oil; $\delta_{\mathrm{H}}$ $\left(500 \mathrm{MHz}, \mathrm{CDCl}_{3}\right) 1.07\left[9 \mathrm{H}, \mathrm{s}, \mathrm{SiC}\left(\mathrm{CH}_{3}\right)_{3}\right], 1.31(3 \mathrm{H}, \mathrm{t}, J$ 7.0, $\left.\mathrm{OCH}_{2} \mathrm{CH}_{3}\right), 2.63\left(2 \mathrm{H}, \mathrm{t}, \mathrm{J} 6.4,4-\mathrm{H}_{2}\right), 3.72\left(3 \mathrm{H}, \mathrm{s}, \mathrm{OCH}_{3}\right), 3.86(2 \mathrm{H}$, $\mathrm{t}$, J 6.7, 5- $\left.\mathrm{H}_{2}\right), 4.32\left(2 \mathrm{H}, \mathrm{q}, J 7.3, \mathrm{OCH}_{2} \mathrm{CH}_{3}\right), 6.10(1 \mathrm{H}, \mathrm{s}, 2-\mathrm{H})$, 7.37-7.47 $(6 \mathrm{H}, \mathrm{m}, \mathrm{ArH})$ and 7.66-7.70 $(4 \mathrm{H}, \mathrm{m}, \mathrm{ArH}) ; \delta_{\mathrm{F}}(471$ $\left.\mathrm{MHz}, \mathrm{CDCl}_{3}\right)-100.24$.

Methyl (E)-3-(2-tert-butyldiphenylsilyloxyethyl)-4,4difluoro-5,6-dihydroxyhex-2-enoate (66). Acetonitrile $(0.71 \mathrm{~mL})$ and ruthenium(III) chloride hydrate $(16 \mathrm{mg}, 0.062 \mathrm{mmol})$ were added to the alkene $59(0.14 \mathrm{~g}, 0.31 \mathrm{mmol})$ in aqueous EtOAc $(1: 1,1.42 \mathrm{~mL})$. Sodium periodate $(0.066 \mathrm{~g}, 0.31 \mathrm{mmol})$ was added in ten equal portions over $5 \mathrm{~h}$ and the mixture stirred for a further $30 \mathrm{~min}$ before saturated aqueous sodium thiosulfate $(10 \mathrm{~mL})$ and EtOAc $(10 \mathrm{~mL})$ were added. The aqueous phase was extracted with EtOAc $(3 \times 10 \mathrm{~mL})$ and the organic extracts were washed with brine $(40 \mathrm{~mL})$, dried $\left(\mathrm{MgSO}_{4}\right)$ and concentrated under reduced pressure. Chromatography of the residue (1:99 to 50:50 ether:light petroleum) gave recovered alkene 59 (61 $\mathrm{mg}, 44 \%)$ followed by the title compound $66(65 \mathrm{mg}, 0.14$ $\mathrm{mmol}, 44 \%$ ) as a colourless oil, $R_{\mathrm{f}}=0.60$ (ether) (Found: $\mathrm{M}^{+}+\mathrm{Na}$, 501.1898. $\mathrm{C}_{25} \mathrm{H}_{32} \mathrm{O}_{5} \mathrm{~F}_{2} \mathrm{SiNa}$ requires $\left.\mathrm{M}, 501.1885\right) ; v_{\max } / \mathrm{cm}^{-1} 3401$, 2952, 2932, 2889, 2857, 1727, 1428, 1260, 1200, 1105, 1081, 1039,822 and 738; $\delta_{\mathrm{H}}\left(400 \mathrm{MHz}, \mathrm{CDCl}_{3}\right) 1.06\left[9 \mathrm{H}, \mathrm{s}, \mathrm{SiC}\left(\mathrm{CH}_{3}\right)_{3}\right]$, 2.85 and 3.07 (each $\left.1 \mathrm{H}, \mathrm{dt}, J 13.6,6.3,1^{\prime}-\mathrm{H}\right), 3.57(1 \mathrm{H}, \mathrm{br} . \mathrm{s}$, $\mathrm{OH}), 3.69\left(3 \mathrm{H}, \mathrm{s}, \mathrm{OCH}_{3}\right), 3.82\left(2 \mathrm{H}, \mathrm{m}, 6-\mathrm{H}_{2}\right), 3.91\left(2 \mathrm{H}, \mathrm{t}, \mathrm{J} 6.3,2^{\prime}-\right.$ $\left.\mathrm{H}_{2}\right), 4.00(1 \mathrm{H}, \mathrm{m}, 5-\mathrm{H}), 6.30(1 \mathrm{H}, \mathrm{d}, J 2.3,2-\mathrm{H}), 7.37-7.48(6 \mathrm{H}, \mathrm{m}$, $\mathrm{ArH})$ and 7.63-7.70 (4 H, m, $\mathrm{ArH}) ; \delta_{\mathrm{C}}\left(100 \mathrm{MHz} \mathrm{CDCl}_{3}\right) 19.1,26.8$, $30.3,51.6,60.6\left(\mathrm{dd},{ }^{3} J_{\mathrm{C}-\mathrm{F}} 4.4,1.5\right), 63.4,71.8\left(\mathrm{dd},{ }^{2} \mathrm{~J}_{\mathrm{C}-\mathrm{F}} 32.4,25.8\right)$, $120.6\left(\mathrm{dd},{ }^{1} J_{\mathrm{C}-\mathrm{F}} 254.1,247.5\right), 122.3$ (dd, $\left.{ }^{3} J_{\mathrm{C}-\mathrm{F}} 11.1,8.8\right), 127.8$, 
$129.9,132.8,132.9,135.5,135.6,149.0\left(t,{ }^{2} J_{C-F} 22.1\right)$ and 165.4; $\delta_{\mathrm{F}}\left(377 \mathrm{MHz}, \mathrm{CDCl}_{3}\right)-117.88$ and -107.56 (each d, $\left.{ }^{2} J_{\mathrm{F}-\mathrm{F}} 249.0\right)$; $\mathrm{m} / \mathrm{z}\left(\mathrm{ES}^{+}\right) 501.3\left(\mathrm{M}^{+}+23,100 \%\right)$.

\section{Methyl (E)-3-(2-tert-butyldiphenylsilyloxyethyl)-4,4-} difluoro-5-hydroxy-6,6-dimethylocta-2,7-dienoate (68). Solid sodium carbonate $(39 \mathrm{mg}, 0.37 \mathrm{mmol}$ ) and lead(IV) acetate (65 $\mathrm{mg}, 0.15 \mathrm{mmol}$ ) were added to the diol $66(59 \mathrm{mg}, 0.12 \mathrm{mmol})$ in DCM $(1.2 \mathrm{~mL})$. The mixture was stirred at $\mathrm{rt}$ for $30 \mathrm{~min}$, filtered and concentrated under reduced pressure to give the aldehyde 67 that was azeotroped with benzene. 1-Bromo-3methylbut-2-ene $(0.14 \mathrm{~mL}, 1.23 \mathrm{mmol})$ was added to this aldehyde in THF $(2.4 \mathrm{~mL})$ and the solution was added to a suspension of zinc dust ( $80 \mathrm{mg}, 1.23 \mathrm{mmol})$ and titanocene dichloride $(3 \mathrm{mg}, 0.012 \mathrm{mmol})$ in THF $(2.45 \mathrm{~mL})$ that had been pre-stirred for $5 \mathrm{~min}$. The mixture was stirred for $2.5 \mathrm{~h}$ before aqueous hydrogen chloride $(10 \%, 10 \mathrm{~mL})$ and ether $(10 \mathrm{~mL}$ were added. The aqueous phase was extracted with ether $(3 \times 30 \mathrm{~mL})$ and the organic extracts were washed with brine $(40 \mathrm{~mL})$, dried $\left(\mathrm{MgSO}_{4}\right)$ and concentrated under reduced pressure. Chromatography of the residue (10:90 ether:light petroleum) gave the title compound 68 (41 $\mathrm{mg}, 0.079 \mathrm{mmol}, 65 \%)$ as a colourless oil, $R_{\mathrm{f}}=0.40$ (30:70 ether:light petroleum) (Found: $\mathrm{M}^{+}$ $+\mathrm{Na}, 539.2392 . \mathrm{C}_{29} \mathrm{H}_{38} \mathrm{O}_{4} \mathrm{~F}_{2} \mathrm{SiNa}$ requires $\left.\mathrm{M}, 539.2405\right) ; v_{\text {max }} / \mathrm{cm}^{-1}$ $3495,3071,2957,2932,2889,2858,1727,1428,1261,1202$, 1110, 1072, 916, 823 and 740; $\delta_{\mathrm{H}}\left(400 \mathrm{MHz}, \mathrm{CDCl}_{3}\right) 1.05[9 \mathrm{H}, \mathrm{s}$, $\left.\mathrm{SiC}\left(\mathrm{CH}_{3}\right)_{3}\right], 1.16\left(3 \mathrm{H}, \mathrm{s}, 6-\mathrm{CH}_{3}\right), 1.18\left(3 \mathrm{H}, \mathrm{d}, J 2.0,6-\mathrm{CH}_{3}\right), 2.80(1$ $\mathrm{H}$, br. s, OH), 2.85 and 3.06 (each $1 \mathrm{H}, \mathrm{dt}, J 13.4,6.8,1^{\prime}-\mathrm{H}$ ), 3.60 $(1 \mathrm{H}, \mathrm{d}, \mathrm{J} 23.4,5-\mathrm{H}), 3.68\left(3 \mathrm{H}, \mathrm{s}, \mathrm{OCH}_{3}\right), 3.82-3.90\left(2 \mathrm{H}, \mathrm{m}, 2^{\prime}-\mathrm{H}_{2}\right)$, 5.04-5.11 ( $\left.2 \mathrm{H}, \mathrm{m}, 8-\mathrm{H}_{2}\right), 5.99(1 \mathrm{H}, \mathrm{ddq}, J 17.4,10.8,1.2,7-\mathrm{H})$, 6.23 (1 H, d, J 2.8, 2-H), 7.36-7.47 (6 H, m, ArH) and 7.65-7.70 (4 $\mathrm{H}, \mathrm{m}, \mathrm{ArH}) ; \delta_{\mathrm{C}}\left(125 \mathrm{MHz}, \mathrm{CDCl}_{3}\right) 19.1,24.1$ (t, $\left.{ }^{4} J_{\mathrm{C}-\mathrm{F}} 3.6\right), 24.2$, 26.8, 30.9, 41.0, 51.5, 63.3, 76.5 (dd, $\left.{ }^{2} J_{C-F} 30.9,26.4\right), 113.3$, $121.4\left(\mathrm{dd},{ }^{3} J_{\mathrm{C}-\mathrm{F}} 11.8,8.2\right), 122.7\left(\mathrm{dd},{ }^{1} J_{\mathrm{C}-\mathrm{F}} 259.3,249.3\right), 127.7$, 129.7(2), 133.1(2), 135.6(2), $144.1\left(\mathrm{~d},{ }^{4} J_{C-F} 1.8\right), 150.7\left(t,{ }^{2} J_{C-F}\right.$ 21.9) and 165.7; $\delta_{\mathrm{F}}\left(377 \mathrm{MHz}, \mathrm{CDCl}_{3}\right)-115.62$ and -99.51 (each d, $\left.{ }^{2} J_{F-F} 245.2\right) ; m / z\left(E S^{+}\right) 539.3\left(\mathrm{M}^{+}+23,100 \%\right)$.

Methyl (E)-3-(2-tert-butyldiphenylsilyloxyethyl)-4,4difluoro-6,6-dimethyl-5-oxo-octa-2,7-dienoate (69). NMethylmorpholine- $N$-oxide (11 mg, $0.09 \mathrm{mmol}$ ) and TPAP (1 mg, $0.003 \mathrm{mmol}$ ) were added to a stirred suspension of the alcohol 68 (31 mg, $0.06 \mathrm{mmol}$ ) and $4 \AA$ molecular sieves (36 mg) in DCM $(0.78 \mathrm{~mL})$ and the mixture stirred at $\mathrm{rt}$ for $30 \mathrm{~min}$ then concentrated under reduced pressure. Chromatography of the residue (2:98 to $10: 90$ ether:light petroleum) gave the title compound 69 (27 mg, $0.053 \mathrm{mmol}, 87 \%)$ as a colourless oil, $R_{\mathrm{f}}=$ 0.48 (10:90 ether:light petroleum) (Found: $\mathrm{M}^{+}+\mathrm{Na}, 537.2264$. $\mathrm{C}_{29} \mathrm{H}_{36} \mathrm{O}_{4} \mathrm{~F}_{2} \mathrm{SiNa}$ requires $\left.\mathrm{M}, 537.2249\right) ; v_{\max } / \mathrm{cm}^{-1} 2932,2857$, $1729,1428,1261,1201,1109,1039,919,823$ and $740 ; \delta_{H}(500$ $\left.\mathrm{MHz}, \mathrm{CDCl}_{3}\right) 1.03\left[9 \mathrm{H}, \mathrm{s}, \mathrm{SiC}\left(\mathrm{CH}_{3}\right)_{3}\right], 1.33\left(6 \mathrm{H}, \mathrm{s}, 2 \times 6-\mathrm{CH}_{3}\right), 2.96$ $\left(2 \mathrm{H}, \mathrm{t}, J \mathrm{~J} .3,1^{\prime}-\mathrm{H}_{2}\right), 3.69\left(3 \mathrm{H}, \mathrm{s}, \mathrm{OCH}_{3}\right), 3.74\left(1 \mathrm{H}, \mathrm{t}, J \mathrm{~J} .3,2^{\prime}-\mathrm{H}_{2}\right)$, $5.15(1 \mathrm{H}, \mathrm{d}, J 17.4,8-\mathrm{H}), 5.19\left(1 \mathrm{H}, \mathrm{d}, J 10.8,8-\mathrm{H}^{\prime}\right), 5.95(1 \mathrm{H}$, $\mathrm{ddt}, J$ 17.4, 10.8, 1.0, 7-H), $6.18(1 \mathrm{H}, \mathrm{t}, J$ 1.6, 2-H), 7.36-7.45 (6 $\mathrm{H}, \mathrm{m}, \mathrm{ArH})$ and 7.65-7.69 $(4 \mathrm{H}, \mathrm{m}, \mathrm{ArH}) ; \delta_{\mathrm{c}}\left(125 \mathrm{MHz}^{\mathrm{C}} \mathrm{CDCl}_{3}\right)$ 19.1, $24.0\left(t,{ }^{4} J_{C-F} 1.8\right), 26.7,30.5,49.7,51.6,62.1,116.1,116.7$ $\left(t,{ }^{1} J_{C-F} 257.5\right), 123.3\left(t,{ }^{3} J_{C-F} 9.9\right), 127.6,129.5,133.6,135.6$,
139.7, $146.7\left(\mathrm{t},{ }^{2} J_{\mathrm{C}-\mathrm{F}} 21.7\right), 165.0$ and $200.5\left(\mathrm{t},{ }^{2} J_{\mathrm{C}-\mathrm{F}} 28.9\right) ; \delta_{\mathrm{F}}(471$ $\left.\mathrm{MHz}, \mathrm{CDCl}_{3}\right)-102.04 ; \mathrm{m} / \mathrm{z}\left(\mathrm{ES}^{+}\right) 537\left(\mathrm{M}^{+}+23,100 \%\right), 437(20)$ and 259 (80).

\section{3,3-Difluoro-2-hydroxy-2-(3-methylbut-1-en-3-yl)-4-[(E)-} methoxycarbonylmethylene]tetrahydro-4H-pyran (70). Hydrogen fluoride.pyridine complex $(70 \% \mathrm{HF}, 0.2 \mathrm{~mL})$ was added rapidly to the ketone $69(34 \mathrm{mg}, 0.066 \mathrm{mmol})$ and pyridine $(0.39 \mathrm{~mL})$ in THF $(3.9 \mathrm{~mL})$ at $0{ }^{\circ} \mathrm{C}$ and the mixture stirred for $2.5 \mathrm{~h}$. Saturated aqueous sodium bicarbonate $(10 \mathrm{~mL})$ was added and the mixture was stirred at $\mathrm{rt}$ for $1 \mathrm{~h}$. After the addition of EtOAc $(10 \mathrm{~mL})$, the aqueous phase was extracted with EtOAc $(3 \times 10 \mathrm{~mL})$ and the organic extracts were washed with brine $(40 \mathrm{~mL})$, dried $\left(\mathrm{MgSO}_{4}\right)$ and concentrated under reduced pressure. Chromatography of the residue (10:90 ether:light petroleum) gave the title compound 70 (15 mg, 0.054 $\mathrm{mmol}, 82 \%)$ as a colourless oil which solidified on cooling, $R_{\mathrm{f}}=$ 0.31 (30:70 ether:light petroleum), m.p. $54-56{ }^{\circ} \mathrm{C}$ (Found: $\mathrm{C}$, 56.61; $\mathrm{H}, 6.64$. $\mathrm{C}_{13} \mathrm{H}_{18} \mathrm{O}_{4} \mathrm{~F}_{2}$ requires $\mathrm{C}, 56.50 ; \mathrm{H}, 6.60 \%$; Found: $\mathrm{M}^{+}$ $+\mathrm{Na}$, 299.1071. $\mathrm{C}_{13} \mathrm{H}_{18} \mathrm{O}_{4} \mathrm{~F}_{2} \mathrm{Na}$ requires $\left.\mathrm{M}, 299.1071\right) ; v_{\max } / \mathrm{cm}^{-1}$ $3515,2956,2918,2850,1729,1127$ and 1061: $\delta_{\mathrm{H}}(400 \mathrm{MHz}$, benzene- $\left.d_{6}\right) 1.28\left(3 \mathrm{H}, \mathrm{d}, J 4.0,3^{\prime}-\mathrm{CH}_{3}\right.$ or $\left.4^{\prime}-\mathrm{H}_{3}\right), 1.37(3 \mathrm{H}, \mathrm{d}, J$ $2.5,4^{\prime}-\mathrm{H}_{3}$ or $\left.3^{\prime}-\mathrm{CH}_{3}\right), 2.41(1 \mathrm{H}, \mathrm{dd}, J 13.4,6.6,5-\mathrm{H}), 2.46(1 \mathrm{H}, \mathrm{d}, J$ 3.5, OH), $3.24\left(3 \mathrm{H}, \mathrm{s}, \mathrm{OCH}_{3}\right), 3.41\left(1 \mathrm{H}, \mathrm{dd}, \mathrm{J} 11.1,6.6,5-\mathrm{H}^{\prime}\right), 3.76$ and 3.97 (each $1 \mathrm{H}, \mathrm{m}, 6-\mathrm{H}), 4.82-4.93\left(2 \mathrm{H}, \mathrm{m}, 1^{\prime}-\mathrm{H}_{2}\right), 6.06(1 \mathrm{H}$, $\left.\mathrm{dd}, J \mathrm{~J} .9,10.6,2^{\prime}-\mathrm{H}\right)$ and $6.44(1 \mathrm{H}, \mathrm{dd}, J \mathrm{~J} .0,2.3,4-\mathrm{CH}) ; \delta_{\mathrm{C}}(125$ $\mathrm{MHz}$, benzene- $\left.d_{6}\right) 21.8$ and 24.1 (each $\left.d,{ }^{4} J_{\mathrm{C}-\mathrm{F}} 6.3\right), 28.6\left(\mathrm{~d},{ }^{3} J_{\mathrm{C}-\mathrm{F}}\right.$ 2.7), 46.5, 51.3, 60.5, 99.1 (dd, $\left.{ }^{2} J_{C-F} 30.1,24.4\right), 115.4,117.4$ (dd, $\left.{ }^{3} J_{C-F} 13.5,6.3\right), 118.4\left(d d,{ }^{1} J_{C-F} 263.6,243.7\right), 144.5,148.8\left(d d,{ }^{2} J_{C-}\right.$ F $20.8,18.1)$ and $166.3 ; \delta_{F}\left(377 \mathrm{MHz}\right.$, benzene- $\left.d_{6}\right)-119.61$ and -105.38 (each d, $\left.{ }^{2} J_{F-F} 241.5\right) ; m / z\left(E S^{+}\right) 299.1\left(\mathrm{M}^{+}+23,100 \%\right)$.

(3R,5R,6R)-3,6-Bis(tert-butyldimethylsilyloxy)-5-(2trimethylsilylethoxy)methoxyheptanal (72). A stream of ozone was bubbled through the alkene $71(0.30 \mathrm{~g}, 0.58 \mathrm{mmol})$ in DCM $\left(6.3 \mathrm{~mL}\right.$ ) at $-78{ }^{\circ} \mathrm{C}$ for $5 \mathrm{~min}$ until a blue colour persisted. The system was purged with oxygen for $10 \mathrm{~min}$, triphenylphosphine $(0.31 \mathrm{~g}, 1.16 \mathrm{mmol})$ was added and the mixture was stirred at r.t. for 3 h. Chromatography of the residue (2:98 to $3: 97$ ether:light petroleum) gave the title compound $72(0.29 \mathrm{~g}, 0.56$ mmol, 96\%), as a colourless oil, $R_{\mathrm{f}}=0.23$ (10:90 ether:light petroleum) (Found: $\mathrm{M}^{+}+\mathrm{Na}, 543.3352 . \mathrm{C}_{25} \mathrm{H}_{56} \mathrm{O}_{5} \mathrm{Si}_{3} \mathrm{Na}$ requires M, 543.3333); $v_{\max } / \mathrm{cm}^{-1} 2954,2928,2887,2857,1726,1472$, $1434,1250,1102,1054,1033,810,774$ and $742 ; \delta_{\mathrm{H}}(400 \mathrm{MHz}$, $\left.\mathrm{CDCl}_{3}\right) 0.03\left(9 \mathrm{H}, \mathrm{s}, 3 \times \mathrm{SiCH}_{3}\right), 0.06,0.07,0.08$ and 0.10 (each 3 $\left.\mathrm{H}, \mathrm{s}, \mathrm{SiCH}_{3}\right), 0.88$ and 0.89 [each $9 \mathrm{H}, \mathrm{s}, \mathrm{SiC}\left(\mathrm{CH}_{3}\right)_{3}$ ] , 0.90-1.01 $(2 \mathrm{H}$, $\left.\mathrm{m}, \mathrm{SiCH}_{2}\right), 1.08\left(3 \mathrm{H}, \mathrm{d}, J\right.$ 6.3, 7- $\left.\mathrm{H}_{3}\right), 1.55(1 \mathrm{H}, \mathrm{m}, 4-\mathrm{H}), 1.93(1 \mathrm{H}$, $\mathrm{ddd}, J$ 14.4, 7.8, 2.8, 4- $\left.\mathrm{H}^{\prime}\right), 2.53(1 \mathrm{H}, \mathrm{ddd}, J$ 15.6, 5.0, 2.0, 2-H), $2.65(1 \mathrm{H}$, ddd, J 15.9, 5.6, 2.8, 2-H'), 3.49-3.57 (2 H, m, 5-H, $\mathrm{OHCHCH}_{2} \mathrm{Si}$ ), 3.67 (1 H, ddd, J 11.3, 9.6, 5.6, $\mathrm{OHCHCH}_{2} \mathrm{Si}$ ), 4.05 (1 $\mathrm{H}, \mathrm{m}, 6-\mathrm{H}), 4.35(1 \mathrm{H}, \mathrm{m}, 3-\mathrm{H}$ ), 4.69 and 4.73 (each $1 \mathrm{H}, \mathrm{d}, \mathrm{J}$ 6.9, $\mathrm{OHCHO})$ and $9.84(1 \mathrm{H}, \mathrm{t}, J 2.8,1-\mathrm{H}) ; \delta_{\mathrm{C}}\left(100 \mathrm{MHz} \mathrm{CDCl}_{3}\right)-4.8$, $-4.7,-4.4,-4.3,-1.5,17.0,17.9,18.0,18.1,25.8(2), 36.8,51.7$, $65.5,66.2,68.8,79.7,95.7$ and 202.3; $\mathrm{m} / \mathrm{z}\left(\mathrm{ES}^{-}\right) 557.4$ ([M + 37]', $50 \%)$ and $555.4([\mathrm{M}+35],, 100)$. 
(6S,8R,9R)-6,9-Bis(tert-butyldimethylsilyloxy)-3,3-difluoro8-(2-trimethylsilylethoxy)methoxydec-1-en-4-ol (73). Indium powder (49 $\mathrm{mg}, 0.42 \mathrm{mmol}$ ) and 3-bromo-3,3-difluoroprop-1ene 37 (66 $\mathrm{mg}, 0.42 \mathrm{mmol}$ ) were added to a vigorously stirred solution of the aldehyde $\mathbf{7 2}$ (from $73 \mathrm{mg}$ of alkene 71) in DMF $(0.71 \mathrm{~mL})$ and the stirring continued for $4 \mathrm{~h}$. Saturated aqueous ammonium chloride $(10 \mathrm{~mL})$ and EtOAc $(10 \mathrm{~mL})$ were added and the aqueous phase was extracted with EtOAc $(3 \times 10 \mathrm{~mL})$. The organic extracts were washed with brine $(40 \mathrm{~mL})$, dried $\left(\mathrm{MgSO}_{4}\right)$ and concentrated under reduced pressure. Chromatography of the residue (1:99 to 5:95 ether:light petroleum) gave the title compound 73 (56 mg, $0.094 \mathrm{mmol}, 66 \%$ from alkene 71), as a colourless oil, a 53:47 mixture in favour of the less polar diastereoisomer ( $\left.{ }^{19} \mathrm{~F} \quad \mathrm{NMR}\right), \quad R_{\mathrm{f}}=0.19 \quad(10: 90$ ether:light petroleum) (Found: $\mathrm{M}^{+}+\mathrm{Na}, 621.3619 . \mathrm{C}_{28} \mathrm{H}_{60} \mathrm{O}_{5} \mathrm{~F}_{2} \mathrm{Si}_{3} \mathrm{Na}$ requires $\mathrm{M}, 621.3614) ; v_{\max } / \mathrm{cm}^{-1} 2954,2929,2858,1472,1420,1250$, 1101, 1055, 1032 and 1004; $\mathrm{m} / \mathrm{z}\left(\mathrm{ES}^{+}\right) 621.4\left(\mathrm{M}^{+}+23,30 \%\right)$, $599.4\left(\mathrm{M}^{+}+1,20\right)$ and $481.3(100)$. Repeated chromatography gave a sample of the less polar diastereoisomer; $\delta_{\mathrm{H}}(400 \mathrm{MHz}$, $\left.\mathrm{CDCl}_{3}\right) 0.03\left(9 \mathrm{H}, \mathrm{s}, 3 \times \mathrm{SiCH}_{3}\right), 0.06,0.07,0.11$ and 0.12 (each 3 $\left.\mathrm{H}, \mathrm{s}, \mathrm{SiCH}_{3}\right), 0.89$ and 0.90 [each $9 \mathrm{H}, \mathrm{s}, \mathrm{SiC}\left(\mathrm{CH}_{3}\right)_{3}$ ], 0.91-0.97 $(2 \mathrm{H}$, m, $\left.\mathrm{SiCH}_{2}\right), 1.10\left(3 \mathrm{H}, \mathrm{d}, J\right.$ 6.0, 10- $\left.\mathrm{H}_{3}\right), 1.67(1 \mathrm{H}, \mathrm{m}, 7-\mathrm{H}), 1.75-1.89$ $\left(2 \mathrm{H}, \mathrm{m}, 5-\mathrm{H}_{2}\right), 1.98\left(1 \mathrm{H}, \mathrm{ddd}, \mathrm{J} 14.4,6.0,3.0,7-\mathrm{H}^{\prime}\right), 3.45(1 \mathrm{H}, \mathrm{m}$, 8-H), 3.53 and 3.66 (each $1 \mathrm{H}, \mathrm{m}, \mathrm{OHCHCH}_{2} \mathrm{Si}$ ), $3.84(2 \mathrm{H}, \mathrm{d}, \mathrm{J} 2.0$, 4-OH), $4.05(1 \mathrm{H}, \mathrm{m}, 9-\mathrm{H}), 4.16-4.27(2 \mathrm{H}, \mathrm{m}, 4-\mathrm{H}, 6-\mathrm{H}), 4.67$ and 4.71 (each $1 \mathrm{H}, \mathrm{d}, J$ 7.1, OHCHO), $5.52(1 \mathrm{H}, \mathrm{d}, J 11.1,1-\mathrm{H}), 5.71$ $\left(1 \mathrm{H}, \mathrm{m}, 1-\mathrm{H}^{\prime}\right)$ and $6.03(1 \mathrm{H}, \mathrm{m}, 2-\mathrm{H}) ; \delta_{\mathrm{C}}\left(125 \mathrm{MHz}, \mathrm{CDCl}_{3}\right)-4.8$, $-4.7(2),-4.5,-1.5,17.0,17.9,18.0,18.1,25.8,25.9,35.0,35.2$, 65.4, 69.0, 69.7, $70.7\left(\mathrm{t},{ }^{2} J_{\mathrm{C}-\mathrm{F}} 29.8\right), 80.0,95.6,119.9\left(\mathrm{dd},{ }^{1} J_{\mathrm{C}-\mathrm{F}}\right.$ $239.2,241.9), 120.6\left(t,{ }^{3} J_{C-F} 9.0\right)$ and $130.4\left(t,{ }^{2} J_{C-F} 25.3\right) ; \delta_{F}(377$ $\left.\mathrm{MHz}, \mathrm{CDCl}_{3}\right)-114.86\left(\mathrm{~d},{ }^{2} J_{\mathrm{F}-\mathrm{F}} 249.7\right)$ and -108.30 (d, ${ }^{2} J_{\mathrm{F}-\mathrm{F}} 248.3$ ). Further elution gave the more polar diastereoisomer; $\delta_{H}(400$ $\left.\mathrm{MHz}, \mathrm{CDCl}_{3}\right) 0.02\left(9 \mathrm{H}, \mathrm{s}, 3 \times \mathrm{SiCH}_{3}\right), 0.06,0.07,0.09$ and 0.10 (each $\left.3 \mathrm{H}, \mathrm{s}, \mathrm{SiCH}_{3}\right), 0.89$ and 0.90 [each $9 \mathrm{H}, \mathrm{s}, \mathrm{SiC}\left(\mathrm{CH}_{3}\right)_{3}$ ], 0.91$0.97\left(2 \mathrm{H}, \mathrm{m}, \mathrm{SiCH}_{2}\right), 1.08\left(3 \mathrm{H}, \mathrm{d}, J \mathrm{~J} 6.3,10-\mathrm{H}_{3}\right), 1.62-1.72(2 \mathrm{H}, \mathrm{m}$, 7-H, 5-H), 1.87-1.97 (2 H, m, 7- $\left.\mathrm{H}^{\prime}, 5-\mathrm{H}^{\prime}\right), 3.47-3.58(3 \mathrm{H}, \mathrm{m}, 8-\mathrm{H}$, $\left.\mathrm{OHCHCH}_{2} \mathrm{Si}, 4-\mathrm{OH}\right), 3.67\left(1 \mathrm{H}, \mathrm{m}, \mathrm{OHCHCH}_{2} \mathrm{Si}\right), 3.99-4.12(3 \mathrm{H}, \mathrm{m}$, 4-H, 6-H, 9-H), 4.69 and 4.73 (each $1 \mathrm{H}, \mathrm{d}, \mathrm{J}$ 6.8, OHCHO), 5.52 (1 $\mathrm{H}, \mathrm{d}, J 11.1,1-\mathrm{H}), 5.71\left(1 \mathrm{H}, \mathrm{d}, J 17.4,1-\mathrm{H}^{\prime}\right)$ and $6.02(1 \mathrm{H}, \mathrm{m}, 2-$ $\mathrm{H}) ; \delta_{\mathrm{C}}\left(100 \mathrm{MHz}, \mathrm{CDCl}_{3}\right)-4.8(2),-4.6,-4.3,-1.5,16.9,17.9$, 18.0, 18.1, 25.8(2), 36.0, $36.8\left(\mathrm{t},{ }^{3} J_{\mathrm{C}-\mathrm{F}} 2.2\right), 65.7,68.7,69.9,70.9$ $\left(t,{ }^{2} J_{C-F} 29.9\right), 80.0,95.3,119.9\left(d d,{ }^{1} J_{C-F} 239.9,242.1\right), 120.6(t$, $\left.{ }^{3} J_{\text {C-F }} 8.8\right)$ and $130.3\left(t,{ }^{2} J_{C-F} 25.5\right) ; \delta_{F}\left(377 \mathrm{MHz} \mathrm{CDCl}_{3}\right)-114.45$ and -108.07 (both $d,{ }^{2} J_{F-F} 248.3$ ).

(6R,8R,9R)-6,9-Bis(tert-butyldimethylsilyloxy)-3,3-difluoro8-(2-trimethylsilylethoxy)methoxydec-1-en-4-one (74). NMethylmorpholine- $N$-oxide $(19 \mathrm{mg}, 0.16 \mathrm{mmol}$ ) and TPAP (4 mg, $0.011 \mathrm{mmol}$ ) were added to a suspension of a mixture of the diastereoisomers of the alcohol $73(63 \mathrm{mg}, 0.105 \mathrm{mmol})$ and $4 \AA$ molecular sieves $(65 \mathrm{mg})$ in DCM $(1.1 \mathrm{~mL})$. The mixture stirred at $\mathrm{rt}$ for $16 \mathrm{~h}$ then concentrated under reduced pressure. Chromatography of the residue $0.5: 99.5$ to $5: 95$ ether:light petroleum) gave the title compound 74 as a colourless oil (47 $\mathrm{mg}, 0.079 \mathrm{mmol}, 75 \%), R_{\mathrm{f}}=0.59$ (10:90 ether:light petroleum) (Found: $\mathrm{M}^{+}+\mathrm{Na}$, 619.3461. $\mathrm{C}_{28} \mathrm{H}_{58} \mathrm{O}_{5} \mathrm{~F}_{2} \mathrm{Si}_{3} \mathrm{Na}$ requires $\mathrm{M}$, 619.3458); $v_{\max } / \mathrm{cm}^{-1} 2954,2929,2887,2858,1746,1472,1379$,
$1250,1099,1055,1033,1004,938,859,831,810$ and $774 ; \delta_{H}$ $\left(400 \mathrm{MHz}, \mathrm{CDCl}_{3}\right) 0.02\left(9 \mathrm{H}, \mathrm{s}, 3 \times \mathrm{SiCH}_{3}\right), 0.03,0.06(2)$ and 0.10 (each $3 \mathrm{H}, \mathrm{s}, \mathrm{SiCH}_{3}$ ), 0.85 and 0.88 [each $9 \mathrm{H}, \mathrm{s}, \mathrm{SiC}\left(\mathrm{CH}_{3}\right)_{3}$ ], 0.91$1.01\left(2 \mathrm{H}, \mathrm{m}, \mathrm{SiCH}_{2}\right), 1.07\left(3 \mathrm{H}, \mathrm{d}, \mathrm{J} 6.3,10-\mathrm{H}_{3}\right), 1.53(1 \mathrm{H}, \mathrm{m}, 7-\mathrm{H})$, $1.84\left(1 \mathrm{H}\right.$, ddd, J 14.4, 6.5, 2.8, 7-H'), 2.84-2.97 (2 H, m, 5- $\left.\mathrm{H}_{2}\right)$, 3.49-3.59 (2 H, m, 8- $\left.\mathrm{H}, \mathrm{OHCHCH}_{2} \mathrm{Si}\right), 3.66\left(1 \mathrm{H}, \mathrm{m}, \mathrm{OHCHCH}_{2} \mathrm{Si}\right)$, $4.03(1 \mathrm{H}, \mathrm{m}, 9-\mathrm{H}), 4.37(1 \mathrm{H}, \mathrm{m}, 6-\mathrm{H}), 4.67$ and 4.71 (each $1 \mathrm{H}, \mathrm{d}$, J 7.1, OHCHO), $5.64(1 \mathrm{H}, \mathrm{d}, J 10.7,1-\mathrm{H}), 5.83\left(1 \mathrm{H}, \mathrm{m}, 1-\mathrm{H}^{\prime}\right)$ and $5.97(1 \mathrm{H}, \mathrm{m}, 2-\mathrm{H}) ; \delta_{\mathrm{C}}\left(100 \mathrm{MHz}, \mathrm{CDCl}_{3}\right)-4.8,-4.7(2),-4.4,-1.5$, $17.1,17.9,18.0,18.1,25.8,25.9,36.8,45.2,65.5,66.0,68.9$, 79.7, 95.5, $114.2\left(t,{ }^{1} J_{C-F} 249.4\right), 122.9\left(t,{ }^{3} J_{C-F} 9.5\right), 128.4\left(t,{ }^{2} J_{C-F}\right.$ 25.1) and $198.1\left(t,{ }^{2} J_{\text {CF }} 31.4\right) ; \delta_{\mathrm{F}}\left(377 \mathrm{MHz}, \mathrm{CDCl}_{3}\right)-108.87$ and -108.03 (each $\left.d,{ }^{2} J_{F-F} 266.0\right) ; m / z\left(E^{+}\right) 619.4\left(\mathrm{M}^{+}+23,100 \%\right)$.

Methyl (5S,7R,8R,2E)-5,8-bis(tert-butyldimethylsilyloxy)-3(1,1-difluoropropenyl)-7-(2-trimethylsilylethoxy)methoxynon-

2-enoate (75). Methoxycarbonylmethylene(triphenyl)phosphorane $(0.12 \mathrm{~g}, 0.36 \mathrm{mmol})$ was added to the ketone $\mathbf{7 4}$ $(0.18 \mathrm{~g}, 0.30 \mathrm{mmol})$ in toluene $(1.5 \mathrm{~mL})$ and the mixture heated at $90{ }^{\circ} \mathrm{C}$ for $16 \mathrm{~h}$. After allowing to cool to $\mathrm{rt}$, the reaction mixture was concentrated under reduced pressure. Chromatography of the residue (1:99 to $2: 98$ ether:light petroleum) gave recovered starting material (18 mg, 10\%) followed by the title compound $75(0.124 \mathrm{~g}, 0.19 \mathrm{mmol}, 64 \%)$ as a clear oil, $R_{\mathrm{f}}=0.37$ (10:90 ether:light petroleum) (Found: $\mathrm{M}^{+}+$ $\mathrm{Na}, 675.3719$. $\mathrm{C}_{31} \mathrm{H}_{62} \mathrm{O}_{6} \mathrm{~F}_{2} \mathrm{Si}_{3} \mathrm{Na}$ requires $\left.\mathrm{M}, 675.3720\right) ; v_{\text {max }} / \mathrm{cm}^{-1}$ 2953, 2929, 2887, 2857, 1732, 1250, 1196, 1178, 1100, 1052, $1033,857,832,810$ and $774 ; \delta_{\mathrm{H}}\left(400 \mathrm{MHz}, \mathrm{CDCl}_{3}\right) 0.02(9 \mathrm{H}, \mathrm{s}, 3$ $\left.\times \mathrm{SiCH}_{3}\right), 0.05,0.06,0.07$ and 0.08 (each $\left.3 \mathrm{H}, \mathrm{s}, \mathrm{SiCH}_{3}\right), 0.87$ and 0.89 [each $9 \mathrm{H}, \mathrm{s}, \mathrm{SiC}\left(\mathrm{CH}_{3}\right)_{3}$ ], 0.90-1.01 $\left(2 \mathrm{H}, \mathrm{m}, \mathrm{SiCH}_{2}\right), 1.05(3 \mathrm{H}$, d, J 6.3, 9- $\left.\mathrm{H}_{3}\right), 1.45(1 \mathrm{H}, \mathrm{m}, 6-\mathrm{H}), 1.73\left(1 \mathrm{H}, \mathrm{dd}, J\right.$ 14.2, 8.3, 6- $\left.\mathrm{H}^{\prime}\right)$, $2.87(1 \mathrm{H}, \mathrm{dd}, J 12.8,6.5,4-\mathrm{H}), 2.98\left(1 \mathrm{H}, \mathrm{dd}, \mathrm{J} 12.8,7.6,4-\mathrm{H}^{\prime}\right)$, 3.47-3.56 (2 H, m, 7-H, OHCHCH $3.74\left(3 \mathrm{H}, \mathrm{s}, \mathrm{OCH}_{3}\right), 4.01(1 \mathrm{H}, \mathrm{m}, 8-\mathrm{H}), 4.12(1 \mathrm{H}, \mathrm{m}, 5-\mathrm{H}), 4.71(2$ $\left.\mathrm{H}, \mathrm{s}, \mathrm{OCH}_{2} \mathrm{O}\right), 5.53\left(1 \mathrm{H}, \mathrm{d}, J 11.0,3^{\prime}-\mathrm{H}\right), 5.71\left(1 \mathrm{H}, \mathrm{d}, J 17.1,3^{\prime}-\right.$ $\left.\mathrm{H}^{\prime}\right), 5.92\left(1 \mathrm{H}, \mathrm{m}, 2^{\prime}-\mathrm{H}\right)$ and $6.20(1 \mathrm{H}, \mathrm{s}, 2-\mathrm{H}) ; \delta_{\mathrm{C}}(100 \mathrm{MHz}$, $\left.\mathrm{CDCl}_{3}\right)-4.8(2),-4.6,-4.1,-1.5,17.3,17.9,18.0,18.1,25.9(2)$, $35.6,36.1,51.5,65.2,68.9,69.1,80.1,95.9,118.9\left(\mathrm{t},{ }^{1} J_{\mathrm{C}-\mathrm{F}} 240.7\right)$, $120.6\left(\mathrm{t},{ }^{3} J_{\mathrm{C}-\mathrm{F}} 8.8\right), 122.0\left(\mathrm{t},{ }^{3} \mathrm{~J}_{\mathrm{C}-\mathrm{F}} 8.0\right), 131.8\left(\mathrm{t},{ }^{2} J_{\mathrm{C}-\mathrm{F}} 28.4\right), 149.4(\mathrm{t}$, $\left.{ }^{2} J_{C-F} 24.8\right)$ and 165.8; $\delta_{F}\left(377 \mathrm{MHz} \mathrm{CDCl}_{3}\right)-99.44$ and -98.74 (each $\left.d,{ }^{2} J_{F-F} 252.4\right) ; m / z\left(E S^{+}\right) 675.4\left(M^{+}+23,50 \%\right)$ and 101.4 (100).

Methyl (5S,7R,8R,2E)-5,8-bis(tert-butyldimethylsilyloxy)-3(1,1-difluoro-2,3-dihydroxypropyl)-7-(2-

(trimethylsilylethoxy)methoxynon-2-enoate (76). Acetonitrile $(0.34 \mathrm{~mL})$ and ruthenium(III) chloride hydrate $(40 \mathrm{mg}, 0.016$ mmol) were added to the alkene $75(51 \mathrm{mg}, 0.078 \mathrm{mmol})$ in a mixture of EtOAc and water $(1: 1,0.34 \mathrm{~mL})$. Sodium periodate ( $20 \mathrm{mg}, 0.094 \mathrm{mmol}$ ) was then added in ten equal portions over $9 \mathrm{~h}$. After stirring for a further $30 \mathrm{~min}$, saturated aqueous sodium sulfite $(5 \mathrm{~mL})$ and EtOAc $(5 \mathrm{~mL})$ were added and the aqueous phase was extracted with EtOAc $(3 \times 5 \mathrm{~mL})$. The organic extracts were washed with brine $(20 \mathrm{~mL})$, dried $\left(\mathrm{MgSO}_{4}\right)$ and concentrated under reduced pressure. Chromatography of the residue (10:90 to 40:60 ether:light petroleum) gave recovered alkene 75 (20 mg, 39\%) followed by the title compound 76 (33 
$\mathrm{mg}, 0.048 \mathrm{mmol}, 61 \%)$, as a colourless oil, a 55:45 mixture of diastereoisomers $\left({ }^{19} \mathrm{~F} \quad \mathrm{NMR}\right), \quad R_{\mathrm{f}}=0.15 \quad$ (50:50 ether:light petroleum) (Found: $\mathrm{M}^{+}+\mathrm{Na}$, 709.3753. $\mathrm{C}_{31} \mathrm{H}_{64} \mathrm{O}_{8} \mathrm{~F}_{2} \mathrm{Si}_{3} \mathrm{Na}$ requires $M, 709.3775) ; v_{\text {max }} / \mathrm{cm}^{-1} 3394,2953,2929,2894,2857,1730$, $1472,1463,1378,1250,1101,1055,1033,834$ and 774; $\delta_{H}(500$ $\left.\mathrm{MHz}, \mathrm{CDCl}_{3}\right) 0.02\left(9 \mathrm{H}, \mathrm{s}, 3 \times \mathrm{SiCH}_{3}\right), 0.05,0.06(2), 0.07$ and 0.08 (each $\left.3 \mathrm{H}, \mathrm{s}, \mathrm{SiCH}_{3}\right), 0.88$ and 0.89 [each $9 \mathrm{H}, \mathrm{s}, \mathrm{SiC}\left(\mathrm{CH}_{3}\right)_{3}$ ], 0.90$1.01\left(2 \mathrm{H}, \mathrm{m}, \mathrm{SiCH}_{2}\right), 1.05\left(3 \mathrm{H}, \mathrm{d}, \mathrm{J} 6.3,9-\mathrm{H}_{3}\right), 1.51(1 \mathrm{H}, \mathrm{m}, 6-\mathrm{H})$, $1.77\left(0.55 \mathrm{H}\right.$, ddd, J 14.5, 7.9, 1.3, 6- $\left.\mathrm{H}^{\prime}\right), 1.85(0.45 \mathrm{H}$, ddd, J 14.5, 6.7, 1.6, 6- $\left.\mathrm{H}^{\prime}\right), 2.23(1 \mathrm{H}$, br. s, OH), $2.78(0.45 \mathrm{H}, \mathrm{dd}, J 12.9,6.3$, 4-H), $2.85(0.55 \mathrm{H}, \mathrm{dd}, J$ 13.6, 7.9, 4-H), $3.14(0.55 \mathrm{H}$, ddd, J 13.6, 6.0, 0.7, 4- $\left.\mathrm{H}^{\prime}\right), 3.20\left(0.45 \mathrm{H}, \mathrm{dd}, \mathrm{J} 12.9,8.2,4-\mathrm{H}^{\prime}\right), 3.46-3.56(2 \mathrm{H}$, $\left.\mathrm{m}, 7-\mathrm{H}, \mathrm{OHCHCH}_{2} \mathrm{Si}\right), 3.67\left(1 \mathrm{H}, \mathrm{m}, \mathrm{OHCHCH}_{2} \mathrm{Si}\right), 3.75(3 \mathrm{H}, \mathrm{s}$, $\left.\mathrm{OCH}_{3}\right)$, 3.78-3.88 (2 H, m, 2'-H, 3'-H), 3.97-4.06 (2 H, m, 8-H, 3'$\left.\mathrm{H}^{\prime}\right), 4.11(0.55 \mathrm{H}, \mathrm{m}, 5-\mathrm{H}), 4.24(0.45 \mathrm{H}, \mathrm{dt}, J 13.3,6.3,5-\mathrm{H}), 4.71$ $(1 \mathrm{H}, \mathrm{d}, J \mathrm{~J} .0, \mathrm{OHCHO}), 4.73(0.45 \mathrm{H}, \mathrm{d}, J \mathrm{~J} .0, \mathrm{OHCHO}), 4.75(0.55$ $\mathrm{H}, \mathrm{d}, J \mathrm{~J} .0, \mathrm{OHCHO})$ and $6.25(1 \mathrm{H}, \mathrm{s}, 2-\mathrm{H}) ; \delta_{\mathrm{C}}\left(125 \mathrm{MHz}, \mathrm{CDCl}_{3}\right)$ $-4.8,-4.7,-4.6,-4.1,-4.0,-1.5,17.1,17.4,18.0(2), 18.1$, $25.9(2), 35.7,36.0,36.5,51.5,60.7\left(t^{3} J_{C-F} 3.6\right), 60.9\left(d d,{ }^{3} J_{C-F} 5.4\right.$, 1.8), 65.4, 65.6, 69.0(2), 70.5, 72.5 (dd, $\left.{ }^{2} J_{C-F} 32.5,27.1\right), 73.1$ (dd, $\left.{ }^{2} J_{C-F} 32.5,25.3\right), 80.5,80.6,95.9,120.3\left(t,{ }^{1} J_{C-F} 248.8\right), 121.0(d d$, $\left.{ }^{1} J_{C-F} 253.3,244.2\right), 122.3$ and 123.6 (each dd, ${ }^{3} J_{C-F} 11.3,7.7$ ), $147.8\left(d d,{ }^{2} J_{C-F} 23.5,20.8\right), 149.5$ (dd, $\left.{ }^{2} J_{C-F} 24.4,19.9\right)$ and $165.6(2) ; \delta_{\mathrm{F}}\left(471 \mathrm{MHz}, \mathrm{CDCl}_{3}\right)-115.11$ (0.55 F, d, $\left.{ }^{2} J_{\mathrm{F}-\mathrm{F}} 249.9\right)$, -113.55 (0.45 F, d, $\left.{ }^{2} J_{F-F} 249.9\right),-107.39\left(0.55 \mathrm{~F}, \mathrm{~d},{ }^{2} J_{F-F} 249.9\right)$ and $-107.28\left(0.45 \mathrm{~F}, \mathrm{~d},{ }^{2} J_{\mathrm{F}-\mathrm{F}} 248.2\right) ; \mathrm{m} / \mathrm{z}\left(\mathrm{ES}^{+}\right) 709.4\left(\mathrm{M}^{+}+23\right.$, $70 \%), 611.2(50)$ and $569.3(100)$.

Methyl (5S,7R,8R,2E)-5,8-bis(tert-butyldimethylsilyloxy)-3(1,1-difluoro-2-hydroxy-3,3-dimethylpent-4-enyl)-7-(2-

trimethylsilylethoxy)methoxynon-2-enoate (77). Solid sodium carbonate $(60 \mathrm{mg}, 0.56 \mathrm{mmol})$ and lead(IV) acetate $(0.10 \mathrm{~g}, 0.22$ $\mathrm{mmol})$ were added to the diol $76(0.13 \mathrm{~g}, 0.186 \mathrm{mmol})$ in dry DCM $(1.9 \mathrm{~mL})$. After stirring at $\mathrm{rt}$ for $90 \mathrm{~min}$, the reaction mixture was filtered through Celite ${ }^{\circledR}$ and concentrated under reduced pressure to give an oil that was dissolved in THF (3.8 $\mathrm{mL})$. 1-Bromo-3-methylbut-2-ene $(0.22 \mathrm{~mL}, 1.86 \mathrm{mmol})$ was added and the solution added to a stirred suspension of zinc dust $(0.12 \mathrm{~g}, 1.86 \mathrm{mmol})$ and titanocene dichloride $(5 \mathrm{mg}, 0.02$ $\mathrm{mmol}$ ) in THF (3.8 mL). After stirring for $16 \mathrm{~h}$, aqueous hydrogen chloride $(10 \%, 20 \mathrm{~mL})$ and EtOAc $(20 \mathrm{~mL})$ were added. The aqueous phase was extracted with EtOAc $(3 \times 20 \mathrm{~mL})$ and the organic extracts were washed with brine $(60 \mathrm{~mL})$, dried $\left(\mathrm{MgSO}_{4}\right)$ and concentrated under reduced pressure. Chromatography of the residue (3:97 to $5: 95$ ether:light petroleum) gave the title compound 77 (94 mg, $0.13 \mathrm{mmol}, 70 \%$ ) as a colourless oil, a 50:50 mixture of diastereoisomers ( $\left.{ }^{19} \mathrm{~F} \mathrm{NMR}\right), R_{\mathrm{f}}=0.49$ (30:70 ether:light petroleum) (Found: $\mathrm{M}^{+}+\mathrm{Na}, 747.4294$. $\mathrm{C}_{35} \mathrm{H}_{70} \mathrm{O}_{7} \mathrm{~F}_{2} \mathrm{Si}_{3} \mathrm{Na}$ requires $\left.\mathrm{M}, 747.4295\right) ; v_{\max } / \mathrm{cm}^{-1} 3449,2954$, 2929, 2894, 2857, 1730, 1472, 1463, 1378, 1362, 1251, 1196, $1180,1151,1103,1071,1057,1033,835$ and $775 ; \delta_{H}(400 \mathrm{MHz}$, $\left.\mathrm{CDCl}_{3}\right) 0.02\left(9 \mathrm{H}, \mathrm{s}, 3 \times \mathrm{SiCH}_{3}\right), 0.06$ and 0.08 (each $6 \mathrm{H}, \mathrm{s}, 2 \times$ $\left.\mathrm{SiCH}_{3}\right), 0.88$ and 0.89 [each $9 \mathrm{H}, \mathrm{s}, \mathrm{SiC}\left(\mathrm{CH}_{3}\right)_{3}$ ], $0.90-0.98(2 \mathrm{H}, \mathrm{m}$, $\mathrm{SiCH}_{2}$ ), 1.03 and 1.05 (each $\left.1.5 \mathrm{H}, \mathrm{d}, J 6.1,9-\mathrm{H}_{3}\right), 1.19$ and 1.21 (each $3 \mathrm{H}, \mathrm{s}, 3^{\prime}-\mathrm{CH}_{3}$ ), 1.50 and 1.75 (each $\left.1 \mathrm{H}, \mathrm{m}, 6-\mathrm{H}\right), 2.68(0.5$ $\mathrm{H}$, br. s, OH), $2.74(0.5 \mathrm{H}$, dd, J 12.8, 6.0, 4-H), $2.85(1 \mathrm{H}, \mathrm{m}, \mathrm{OH}$, 4-H), $3.08\left(0.5 \mathrm{H}, \mathrm{dd}, J 12.8,7.0,4-\mathrm{H}^{\prime}\right), 3.18(0.5 \mathrm{H}, \mathrm{dd}, J 12.8$,
8.6, 4- $\left.\mathrm{H}^{\prime}\right), 3.44-3.56\left(3 \mathrm{H}, \mathrm{m}, 7-\mathrm{H}, 2^{\prime}-\mathrm{H}, \mathrm{OHCHCH} \mathrm{Si}^{2}\right), 3.68(1 \mathrm{H}$, $\left.\mathrm{m}, \mathrm{OHCHCH}_{2} \mathrm{Si}\right), 3.73\left(3 \mathrm{H}, \mathrm{s}, \mathrm{OCH}_{3}\right), 4.04(1.5 \mathrm{H}, \mathrm{m}, 8-\mathrm{H}, 5-\mathrm{H})$, $4.12(0.5 \mathrm{H}, \mathrm{m}, 5-\mathrm{H}), 4.71$ and 4.72 (each $1 \mathrm{H}, \mathrm{d}, J$ 7.0, $\mathrm{OHCHO}$ ), 5.05-5.12 $\left(2 \mathrm{H}, \mathrm{m}, 5^{\prime}-\mathrm{H}_{2}\right), 6.00\left(1 \mathrm{H}, \mathrm{m}, 4^{\prime}-\mathrm{H}\right)$ and 6.16 and 6.21 (each $0.5 \mathrm{H}, \mathrm{m}, 2-\mathrm{H}) ; \delta_{\mathrm{C}}\left(100 \mathrm{MHz} \mathrm{CDCl}_{3}\right)-4.8(3),-4.7,-4.6$, $-4.1,-4.0,-1.5,15.3,17.1,17.2,17.9,18.0,24.0(2), 24.2,24.4$, $25.8,25.9(2), 35.4,35.9,36.0,36.3,41.2,41.3,51.4(2), 65.2$, $65.3,65.8,68.8(2), 69.1,69.7,77.2,78.1$ (dd, $\left.{ }^{2} J_{C-F} 31.4,25.5\right)$, $78.2,80.4,80.7,95.9(2), 112.5,112.9,113.4,113.8,121.8\left(t,{ }^{3} J_{C-F}\right.$ 9.5), $122.4\left(\mathrm{dd},{ }^{1} J_{\mathrm{C}-\mathrm{F}} 253.8,250.9\right), 122.6\left(\mathrm{dd},{ }^{3} J_{\mathrm{C}-\mathrm{F}} 11.7,7.3\right)$, $122.8\left(\mathrm{dd},{ }^{1} J_{\mathrm{C}-\mathrm{F}} 257.4,247.2\right), 144.1,144.3,149.6\left(\mathrm{dd},{ }^{2} J_{\mathrm{C}-\mathrm{F}} 23.3\right.$, 21.1), 151.1 (dd, $\left.{ }^{2} J_{\mathrm{C}-\mathrm{F}} 24.8,21.2\right), 165.8$ and $165.9 ; \delta_{\mathrm{F}}(377 \mathrm{MHz}$, $\left.\mathrm{CDCl}_{3}\right)-113.53\left(0.5 \mathrm{~F}, \mathrm{~d}^{2} \mathrm{~J}_{\mathrm{F}-\mathrm{F}} 246.9\right),-112.33\left(0.5 \mathrm{~F}, \mathrm{~d},{ }^{2} J_{\mathrm{F}-\mathrm{F}}\right.$ 245.6), $-99.26\left(0.5 \mathrm{~F}, \mathrm{~d},{ }^{2} J_{F-F} 245.6\right)$ and $-98.71\left(0.5 \mathrm{~F}, \mathrm{~d},{ }^{2} J_{F-F}\right.$ 245.5); $m / z\left(\mathrm{ES}^{+}\right) 753.6(50 \%), 747.4\left(\mathrm{M}^{+}+23,50\right)$ and 608.4 (100).

Methyl (5S,7R,8R,2E)-5,8-bis(tert-butyldimethylsilyloxy)-3(1,1-difluoro-3,3-dimethyl-2-oxopent-4-enyl)-7-(2trimethylsilylethoxy)methoxynon-2-enoate (78). NMethylmorpholine- $N$-oxide (31 $\mathrm{mg}, 0.265 \mathrm{mmol}$ ) and TPAP (7 $\mathrm{mg}, 0.02 \mathrm{mmol}$ ) were added to a suspension of the alcohol 77 (96 mg, $0.13 \mathrm{mmol}$ ) and $4 \AA$ molecular sieves $(0.11 \mathrm{~g})$ in DCM $(0.89 \mathrm{~mL})$ and the mixture was stirred at $\mathrm{rt}$ for $16 \mathrm{~h}$ then concentrated under reduced pressure. Chromatography of the residue (2:98 to $3: 97$ ether:light petroleum) gave the title compound 78 as a colourless oil $(57 \mathrm{mg}, 0.079 \mathrm{mmol}, 60 \%), R_{\mathrm{f}}=$ 0.44 (10:90 ether:light petroleum) (Found: $\mathrm{M}^{+}+\mathrm{Na}, 745.4164$. $\mathrm{C}_{35} \mathrm{H}_{68} \mathrm{O}_{7} \mathrm{~F}_{2} \mathrm{Si}_{3} \mathrm{Na}$ requires $\left.\mathrm{M}, 745.4139\right) ; v_{\max } / \mathrm{cm}^{-1} 2953,2929$, 2887, 2857, 1731, 1472, 1362, 1250, 1197, 1180, 1101, 1054, $1033,832,809$ and 774; $\delta_{\mathrm{H}}\left(400 \mathrm{MHz}, \mathrm{CDCl}_{3}\right) 0.01(9 \mathrm{H}, \mathrm{s}, 3 \times$ $\left.\mathrm{SiCH}_{3}\right), 0.05(2)$ and $0.07(2)$ (each $\left.3 \mathrm{H}, \mathrm{s}, \mathrm{SiCH}_{3}\right), 0.86$ and 0.88 [each $9 \mathrm{H}, \mathrm{s}, \mathrm{SiC}\left(\mathrm{CH}_{3}\right)_{3}$ ], 0.91-0.97 $\left(2 \mathrm{H}, \mathrm{m}, \mathrm{SiCH}_{2}\right), 1.03(3 \mathrm{H}, \mathrm{d}, J$ 6.1, 9- $\left.\mathrm{H}_{3}\right), 1.35\left(6 \mathrm{H}, \mathrm{s}, 2 \times 3^{\prime}-\mathrm{CH}_{3}\right), 1.41(1 \mathrm{H}, \mathrm{m}, 6-\mathrm{H}), 1.70(1 \mathrm{H}$, $\left.\mathrm{dd}, J 14.2,8.6,6-\mathrm{H}^{\prime}\right), 2.88\left(2 \mathrm{H}, \mathrm{d}, J\right.$ J.1, 4- $\left.\mathrm{H}_{2}\right), 3.47-3.54(2 \mathrm{H}, \mathrm{m}$, $\left.7-\mathrm{H}, \mathrm{OHCHCH}_{2} \mathrm{Si}\right), 3.68\left(1 \mathrm{H}, \mathrm{m}, \mathrm{OHCHCH}_{2} \mathrm{Si}\right), 3.73\left(3 \mathrm{H}, \mathrm{s}, \mathrm{OCH}_{3}\right)$, $4.03(2 \mathrm{H}, \mathrm{m}, 5-\mathrm{H}, 8-\mathrm{H}), 4.70\left(2 \mathrm{H}, \mathrm{s}, \mathrm{OCH}_{2} \mathrm{O}\right), 5.17(1 \mathrm{H}, \mathrm{d}, \mathrm{J} 17.6$, $\left.5^{\prime}-\mathrm{H}\right), 5.22\left(1 \mathrm{H}, \mathrm{d}, J \mathrm{~J} 10.5,5^{\prime}-\mathrm{H}^{\prime}\right), 5.97\left(1 \mathrm{H}, \mathrm{dd}, J 17.4,10.5,4^{\prime}-\mathrm{H}\right)$ and $6.15(1 \mathrm{H}, \mathrm{s}, 2-\mathrm{H}) ; \delta_{\mathrm{C}}\left(125 \mathrm{MHz}, \mathrm{CDCl}_{3}\right)-4.8,-4.7,-4.6,-4.1$, $-1.5,17.2,17.9,18.0,18.1,24.1(2), 25.9(2), 35.7,36.0,49.8$, 51.6, 65.1, 69.0, 69.1, 80.1, 96.0, 116.1, 116.8 (t, $\left.{ }^{1} J_{C-F} 258.2\right)$, $124.0\left(\mathrm{t},{ }^{3} J_{\mathrm{C}-\mathrm{F}} 9.0\right), 139.8,146.8\left(\mathrm{t},{ }^{2} J_{\mathrm{C}-\mathrm{F}} 21.7\right), 165.2$ and $200.4(\mathrm{t}$, $\left.{ }^{2} J_{C-F} 28.9\right) ; \delta_{F}\left(377 \mathrm{MHz}, \mathrm{CDCl}_{3}\right)-101.24$ and -100.50 (each d, ${ }^{2} J_{\mathrm{F}-\mathrm{F}}$ 267.4); $m / z\left(\mathrm{ES}^{+}\right) 745.4\left(\mathrm{M}^{+}+23,100 \%\right)$ and 605.4 (100).

Methyl (5S,7R,8R,2E)-5,8-bis(tert-butyldimethylsilyloxy)-3(1,1-difluoro-3,3-dimethyl-2-oxopent-4-enyl)-7-hydroxynon-2enoate (79). Nitromethane $(0.038 \mathrm{~mL}, 0.70 \mathrm{mmol})$ was added to a suspension of magnesium bromide $(64 \mathrm{mg}, 0.35 \mathrm{mmol})$ in ether $(0.23 \mathrm{~mL})$ followed by the SEM-protected hydroxyketone $78(18 \mathrm{mg}, 0.025 \mathrm{mmol})$ in ether $(0.23 \mathrm{~mL})$, and the mixture stirred at $\mathrm{rt}$ for $2 \mathrm{~h}$. Water $(10 \mathrm{~mL})$ and EtOAc $(10 \mathrm{~mL})$ were added and the aqueous phase extracted with EtOAc $(3 \times 10 \mathrm{~mL})$. The organic extracts were washed with brine $(40 \mathrm{~mL})$, dried $\left(\mathrm{MgSO}_{4}\right)$ and concentrated under reduced pressure. Chromatography of the residue (3:97 ether:light petroleum) gave the title compound 79 (11 $\mathrm{mg}, 0.019 \mathrm{mmol}, 74 \%$ ) as a 
colourless oil, $R_{\mathrm{f}}=0.32$ (10:90 ether:light petroleum) (Found: $[\mathrm{M}-\mathrm{H}]^{-}$, 591.3346. $\mathrm{C}_{29} \mathrm{H}_{53} \mathrm{O}_{6} \mathrm{~F}_{2} \mathrm{Si}_{2}$ requires $\left.\mathrm{M}, 591.3349\right) ; v_{\max } / \mathrm{cm}^{-1}$ 2954, 2930, 2887, 2857, 1731, 1472, 1463, 1363, 1252, 1197, $1179,1071,1036,1005,953,917,872$ and $775 ; \delta_{H}(400 \mathrm{MHz}$, $\left.\mathrm{CDCl}_{3}\right)$ 0.03, 0.06, 0.07 and 0.09 (each $\left.3 \mathrm{H}, \mathrm{s}, \mathrm{SiCH}_{3}\right), 0.87(18 \mathrm{H}$, $\left.\mathrm{s}, 2 \times \mathrm{SiC}\left(\mathrm{CH}_{3}\right)_{3}\right], 1.14\left(3 \mathrm{H}, \mathrm{d}, \mathrm{J} 6.1,9-\mathrm{H}_{3}\right), 1.36\left(6 \mathrm{H}, \mathrm{s}, 2 \times 3^{\prime}-\mathrm{CH}_{3}\right)$, 1.41-1.55 ( $\left.2 \mathrm{H}, \mathrm{m}, 6-\mathrm{H}_{2}\right), 2.28(1 \mathrm{H}, \mathrm{s}, \mathrm{OH}), 2.83(1 \mathrm{H}, \mathrm{dd}, J$ 13.0, 6.4, 4-H), $3.02\left(1 \mathrm{H}, \mathrm{dd}, J\right.$ 12.5, 8.6, 4- $\left.\mathrm{H}^{\prime}\right), 3.50(1 \mathrm{H}, \mathrm{m}, 7-\mathrm{H}), 3.61$ $(1 \mathrm{H}, \mathrm{m}, 5-\mathrm{H}$ or $8-\mathrm{H}), 3.73\left(3 \mathrm{H}, \mathrm{s}, \mathrm{OCH}_{3}\right), 4.20(1 \mathrm{H}, \mathrm{m}, 8-\mathrm{H}$ or $5-$ H), $5.19\left(1 \mathrm{H}, \mathrm{d}, J 17.4,5^{\prime}-\mathrm{H}\right), 5.23\left(1 \mathrm{H}, \mathrm{d}, J\right.$ 10.8, 5'- $\left.\mathrm{H}^{\prime}\right), 5.98(1$ $\left.\mathrm{H}, \mathrm{dd}, J \mathrm{17.4}, 10.8,4^{\prime}-\mathrm{H}\right)$ and $6.14(1 \mathrm{H}, \mathrm{s}, 2-\mathrm{H}) ; \delta_{\mathrm{C}}(125 \mathrm{MHz}$, $\left.\mathrm{CDCl}_{3}\right)-5.0(2),-4.4,-4.2,17.9,18.0,20.1,24.0(2), 25.8,25.9$, $35.4,40.5,49.8\left(\mathrm{t},{ }^{3} \mathrm{~J}_{\mathrm{C}-\mathrm{F}} 1.8\right), 51.6,68.4,71.7,71.8,116.2,116.8$ $\left(t,{ }^{1} J_{C-F} 259.1\right), 123.8\left(t,{ }^{3} J_{C-F} 9.0\right), 139.7,147.4\left(t,{ }^{2} J_{C-F} 20.8\right), 165.2$ and $200.3\left(\mathrm{t},{ }^{2} J_{\mathrm{C}-\mathrm{F}} 28.9\right) ; \delta_{\mathrm{F}}\left(376 \mathrm{MHz}, \mathrm{CDCl}_{3}\right)-101.50$ and -100.00 (each $\left.d,{ }^{2} J_{F-F} 271.5\right) ; m / z\left(E S S^{+}\right) 615.3\left(\mathrm{M}^{+}+23,55 \%\right)$ and $593.3\left(\mathrm{M}^{+}+1,100\right)$.

Methyl $(5 S, 7 R, 8 R, 2 E)-5,8$-bis(tert-butyldimethylsilyloxy)-7butanoyloxy-3-(1,1-difluoro-3,3-dimethyl-2-oxopent-4-

enyl)non-2-enoate (80). 2,4,6-Trichlorobenzoyl chloride $(0.015$ $\mathrm{mL}, 0.098 \mathrm{mmol})$ and triethylamine $(0.03 \mathrm{~mL}, 0.22 \mathrm{mmol})$ were added to butanoic acid $(9 \mu \mathrm{L}, 0.1 \mathrm{mmol})$ in toluene $(0.25 \mathrm{~mL})$ and the solution stirred at $\mathrm{rt}$ for $1.5 \mathrm{~h}$. The alcohol $79(29 \mathrm{mg}$, $0.049 \mathrm{mmol}$ ) and DMAP (6 mg, $0.049 \mathrm{mmol})$ in toluene $(0.5 \mathrm{~mL})$ were added and the solution stirred at $\mathrm{rt}$ for $16 \mathrm{~h}$. Saturated aqueous ammonium chloride $(10 \mathrm{~mL})$ and ether $(10 \mathrm{~mL})$ were added and the aqueous phase extracted with ether $(3 \times 10 \mathrm{~mL})$. The organic extracts were washed with brine $(40 \mathrm{~mL})$, dried $\left(\mathrm{MgSO}_{4}\right)$ and concentrated under reduced pressure. Chromatography of the residue (1:99 to 2:98 ether:light petroleum) gave the title compound $80(26 \mathrm{mg}, 0.039 \mathrm{mmol}$, $80 \%)$ as a colourless oil, $R_{\mathrm{f}}=0.47$ (10:90 ether:light petroleum) (Found: $\mathrm{M}^{+}+\mathrm{H}, 663.3948 . \mathrm{C}_{33} \mathrm{H}_{61} \mathrm{O}_{7} \mathrm{~F}_{2} \mathrm{Si}_{2}$ requires $\mathrm{M}, 663.3924$ ); $v_{\max } / \mathrm{cm}^{-1} 2955,2930,2857,1731,1472,1463,1362,1251,1180$, $1103,1072,1036,939,917,872,835,809$ and $775 ; \delta_{H}(500$ $\left.\mathrm{MHz}, \mathrm{CDCl}_{3}\right) 0.03\left(3 \mathrm{H}, \mathrm{s}, \mathrm{SiCH}_{3}\right), 0.05\left(6 \mathrm{H}, \mathrm{s}, 2 \times \mathrm{SiCH}_{3}\right), 0.07(3$ $\left.\mathrm{H}, \mathrm{s}, \mathrm{SiCH}_{3}\right), 0.87$ and 0.88 [each $9 \mathrm{H}, \mathrm{s}, \mathrm{SiC}\left(\mathrm{CH}_{3}\right)_{3}$ ], $0.94(3 \mathrm{H}, \mathrm{t}, \mathrm{J}$ 7.3, $\left.\mathrm{CH}_{2} \mathrm{CH}_{3}\right), 0.99\left(3 \mathrm{H}, \mathrm{d}, J 6.3,9-\mathrm{H}_{3}\right), 1.36\left(6 \mathrm{H}, \mathrm{s}, 2 \times 3^{\prime}-\mathrm{CH}_{3}\right)$, $1.58(1 \mathrm{H}, \mathrm{m}, 6-\mathrm{H}), 1.63\left(2 \mathrm{H}\right.$, sext, J 7.3, $\left.\mathrm{CH}_{2} \mathrm{CH}_{3}\right), 1.72(1 \mathrm{H}, \mathrm{m}, 6-$ $\left.\mathrm{H}^{\prime}\right), 2.25\left[2 \mathrm{H}, \mathrm{t}, J 7.4, \mathrm{C}(\mathrm{O}) \mathrm{CH}_{2}\right], 2.80(1 \mathrm{H}, \mathrm{dd}, J 13.0,6.1,4-\mathrm{H})$, $2.92\left(1 \mathrm{H}, \mathrm{dd}, \mathrm{J} 13.0,8.5,4-\mathrm{H}^{\prime}\right), 3.75\left(3 \mathrm{H}, \mathrm{s}, \mathrm{OCH}_{3}\right), 3.1-3.96(2 \mathrm{H}$, m, 5-H, 8-H), 4.87 (1 H, ddd, J 10.5, 4.3, 1.4, 7-H), $5.18(1 \mathrm{H}, \mathrm{d}, J$ 17.4, 5'-H), $5.23\left(1 \mathrm{H}, \mathrm{d}, J\right.$ 10.7, 5' $\left.-\mathrm{H}^{\prime}\right), 5.97(1 \mathrm{H}, \mathrm{dd}, J$ 17.4, 10.6, $\left.4^{\prime}-\mathrm{H}\right)$ and $6.15(1 \mathrm{H}, \mathrm{s}, 2-\mathrm{H}) ; \delta_{\mathrm{C}}\left(125 \mathrm{MHz}, \mathrm{CDCl}_{3}\right)-5.1,-4.9,-4.8$, $-4.2,13.8,17.8,17.9(2), 18.3,24.0,24.1,25.8,25.9,34.9,35.7$, $36.4,49.8,51.7,67.5,67.9,73.5,116.2,116.7$ (t, $\left.{ }^{1} J_{C-F} 258.2\right)$, $124.0\left(t,{ }^{3} J_{C-F} 9.0\right), 139.7,146.6\left(t,{ }^{2} J_{C-F} 21.7\right), 165.2,173.0$ and $200.4\left(t,{ }^{2} J_{\text {CF }} 28.9\right) ; \delta_{F}\left(377 \mathrm{MHz}, \mathrm{CDCl}_{3}\right)-101.13$ and -100.29 (each $\left.d,{ }^{2} J_{F-F} 268.8\right) ; m / z\left(E^{+}\right) 685.9\left(M^{+}+23,100 \%\right)$ and 663.4 $\left(\mathrm{M}^{+}+1,70\right)$.

Methyl (5S,7R,8R,2E)-5,8-bis(tert-butyldimethylsilyloxy)-7(2-methylpropanoyloxy)-3-(1,1-difluoro-3,3-dimethyl-2oxopent-4-enyl)non-2-enoate (81). Following the procedure outlined for the synthesis of the ester 80, 2-methylpropanoic acid $(0.038 \mathrm{~mL}, 0.41 \mathrm{mmol})$ in dry toluene $(1.0 \mathrm{~mL}), 2,4,6$ - trichlorobenzoyl chloride $(0.063 \mathrm{~mL}, 0.41 \mathrm{mmol})$, triethylamine $(0.11 \mathrm{~mL}, 0.81 \mathrm{mmol})$, the alcohol $79(0.11 \mathrm{~g}, 0.20 \mathrm{mmol})$ and DMAP (25 mg, $0.20 \mathrm{mmol})$ in toluene $(2.0 \mathrm{~mL})$, after chromatography (1:99 to 2:98 ether:light petroleum), gave the title compound $81(0.125 \mathrm{~g}, 0.189 \mathrm{mmol}, 94 \%)$ as a colourless oil, $R_{\mathrm{f}}=0.54$ (10:90 ether:light petroleum), $[\alpha]_{\mathrm{D}}^{20}=0.1$ (c 1.0, $\mathrm{CHCl}_{3}$ ) (Found: $\mathrm{M}^{+}+\mathrm{H}, 663.3920 . \mathrm{C}_{33} \mathrm{H}_{61} \mathrm{O}_{7} \mathrm{~F}_{2} \mathrm{Si}_{2}$ requires $\mathrm{M}$, 663.3924); $v_{\max } / \mathrm{cm}^{-1} 2954,2929,2856,1731,1472,1251,1195$, $1154,1104,1070,1036,835,809$ and $775 ; \delta_{\mathrm{H}}\left(500 \mathrm{MHz}, \mathrm{CDCl}_{3}\right)$ $0.02,0.04(2)$ and 0.06 (each $3 \mathrm{H}, \mathrm{s}, \mathrm{SiCH}_{3}$ ), 0.86 and 0.87 [each 9 $\left.\mathrm{H}, \mathrm{s}, \mathrm{SiC}\left(\mathrm{CH}_{3}\right)_{3}\right], 0.99\left(3 \mathrm{H}, \mathrm{d}, \mathrm{J} 6.3,9-\mathrm{H}_{3}\right), 1.13$ and 1.14 (each $3 \mathrm{H}$, d, J 7.0, $\left.\mathrm{CHCH}_{3}\right), 1.35\left(6 \mathrm{H}, \mathrm{s}, 2 \times 3^{\prime}-\mathrm{CH}_{3}\right), 1.59(1 \mathrm{H}, \mathrm{ddd}, J$ 14.1, 10.7, 2.9, 6-H), $1.72\left(1 \mathrm{H}\right.$, ddd, J 14.5, 9.5, 1.7, 6- $\left.\mathrm{H}^{\prime}\right), 2.49$ (1 H, hept, J 7.0, 2"-H), $2.79(1 \mathrm{H}, \mathrm{dd}, J 13.1,5.8,4-\mathrm{H}), 2.90(1 \mathrm{H}, \mathrm{dd}, J$ 13.0, 8.6, 4- $\left.\mathrm{H}^{\prime}\right), 3.73\left(3 \mathrm{H}, \mathrm{s}, \mathrm{OCH}_{3}\right), 3.89-3.96(2 \mathrm{H}, \mathrm{m}, 5-\mathrm{H}, 8-\mathrm{H})$, $4.84\left(1 \mathrm{H}\right.$, ddd, J 10.7, 4.1, 1.6, 7-H), $5.17\left(1 \mathrm{H}, \mathrm{d}, J 17.4,5^{\prime}-\mathrm{H}\right)$, $5.21\left(1 \mathrm{H}, \mathrm{d}, J 10.6,5^{\prime}-\mathrm{H}^{\prime}\right), 5.96\left(1 \mathrm{H}, \mathrm{dd}, J 17.4,10.6,4^{\prime}-\mathrm{H}\right)$ and $6.14(1 \mathrm{H}, \mathrm{s}, 2-\mathrm{H}) ; \delta_{\mathrm{C}}\left(125 \mathrm{MHz} \mathrm{CDCl}_{3}\right)-4.9,-4.8,-4.6,-4.0$, $17.9,18.1,19.0,19.1,24.2,24.3,25.9,26.1,34.3,34.9,35.9$, 49.9, 51.8, 67.5, 68.0, 73.4, 116.4, $116.9\left(\mathrm{t},{ }^{1} \mathrm{~J}_{\mathrm{C}-\mathrm{F}} 260.0\right), 124.1(\mathrm{t}$, $\left.{ }^{3} J_{C-F} 9.2\right), 139.9,146.6\left(t,{ }^{2} J_{C-F} 21.8\right), 165.3,176.5$ and $200.6\left(t,{ }^{2} J_{C-}\right.$ F 29.1); $\delta_{\mathrm{F}}\left(376 \mathrm{MHz}, \mathrm{CDCl}_{3}\right)-101.1\left(\mathrm{~d},{ }^{2} J_{\mathrm{F}-\mathrm{F}} 268.0\right)$ and $-100.3(\mathrm{~d}$, $\left.{ }^{2} J_{F-F} 268.7\right) ; m / z\left(E S^{+}\right) 685.4\left(M^{+}+23,85 \%\right)$ and $663.4\left(\mathrm{M}^{+}+1\right.$, 100).

(2S,6S)-3,3-Difluoro-2-hydroxy-6-[(2R,3R)-3-hydroxy-2-(2trimethylsilylethoxy)methoxybutyl]-4-[(E)methoxycarbonylmethylidene]-2-(2-methylbut-3-en-2-

yl)tetrahydro-4H-pyran (82). Hydrogen fluoride-pyridine complex $(0.24 \mathrm{~mL})$ was added to the ketone $78(57 \mathrm{mg}, 0.079$ mmol) and pyridine $(0.4 \mathrm{~mL})$ in $\operatorname{THF}(4.0 \mathrm{~mL})$ at $0{ }^{\circ} \mathrm{C}$ and the mixture stirred for $16 \mathrm{~h}$. Saturated aqueous sodium hydrogen carbonate $(10 \mathrm{~mL})$ was added and the mixture stirred for $1 \mathrm{~h}$. Ethyl acetate $(10 \mathrm{~mL})$ was then added and the aqueous phase was extracted with EtOAc $(3 \times 10 \mathrm{~mL})$. The organic extracts were washed with brine $(40 \mathrm{~mL})$, dried $\left(\mathrm{MgSO}_{4}\right)$ and concentrated under reduced pressure. Chromatography of the residue (0.1:99.9 to 0.2:99.8 methanol:DCM) gave the title compound 82 (26 mg, $0.053 \mathrm{mmol}, 67 \%)$ as a colourless oil, $R_{\mathrm{f}}=0.41$ (4:96 methanol:DCM) (Found: $\mathrm{M}^{+}+\mathrm{Na}, 517.2435 . \mathrm{C}_{23} \mathrm{H}_{40} \mathrm{O}_{7} \mathrm{~F}_{2} \mathrm{SiNa}$ requires $M, 517.2409 ; v_{\max } / \mathrm{cm}^{-1} 3394,2953,1725,1674,1436$, $1370,1280,1250,1202,1178,1033,920,860$ and $836 ; \delta_{H}(500$ $\mathrm{MHz}$, benzene- $\left.d_{6}\right) 0.09\left(9 \mathrm{H}, \mathrm{s}, 3 \times \mathrm{SiCH}_{3}\right), 0.89-1.00(2 \mathrm{H}, \mathrm{m}$, $\mathrm{SiCH}_{2}$ ), $1.25\left(3 \mathrm{H}, \mathrm{d}, J\right.$ 6.0, 4"'- $\left.\mathrm{H}_{3}\right), 1.37$ and 1.42 (each $3 \mathrm{H}, \mathrm{s}, 1^{\prime}-$ $\mathrm{H}_{3}$ or $\left.2^{\prime}-\mathrm{CH}_{3}\right), 1.41-1.52\left(2 \mathrm{H}, \mathrm{m}, 1^{\prime \prime}-\mathrm{H}_{2}\right), 2.30(1 \mathrm{H}, \mathrm{t}, \mathrm{J} 13.1,5-\mathrm{H})$, $2.53(1 \mathrm{H}, \mathrm{s}, \mathrm{OH}), 3.28\left(3 \mathrm{H}, \mathrm{s}, \mathrm{OCH}_{3}\right), 3.45-3.53\left(3 \mathrm{H}, \mathrm{m}, 2^{\prime \prime}-\mathrm{H}, 3^{\prime \prime}-\right.$ $\left.\mathrm{H}, \mathrm{OHCHCH}_{2} \mathrm{Si}\right), 3.68\left(1 \mathrm{H}, \mathrm{m}, \mathrm{OHCHCH}_{2} \mathrm{Si}\right), 3.75(1 \mathrm{H}, \mathrm{d}, J$ J 3.5, $\mathrm{OH}), 4.18(1 \mathrm{H}, \mathrm{dt}, J$ 13.9, 2.2, 5-H'), $4.37(1 \mathrm{H}, \mathrm{br} . \mathrm{t}, \mathrm{J}$ 10.7, 6-H), 4.59 and 4.60 (each $1 \mathrm{H}, \mathrm{d}, J$ 7.3, OHCHO), $5.03(1 \mathrm{H}$, dd, J 10.7, 1.3, 4'-H), $5.07\left(1 \mathrm{H}, \mathrm{d}, J 17.7,1.3,4^{\prime}-\mathrm{H}^{\prime}\right), 6.29(1 \mathrm{H}, \mathrm{dd}, J 17.7$, $\left.10.7,3^{\prime}-\mathrm{H}\right)$ and $6.50\left(1 \mathrm{H}\right.$, dd, J 3.8, 1.9, 4-CH); $\delta_{\mathrm{C}}(125 \mathrm{MHz}$, benzene- $\left.d_{6}\right)-1.3,18.3,19.7,22.8,23.7,34.3,37.6,46.5,51.4$, 67.1, 67.3, 70.7, 85.6, 98.5, 99.4 (dd, $\left.{ }^{2} J_{C-F} 30.7,24.4\right), 114.0$, $117.5\left(\mathrm{dd},{ }^{3} J_{\mathrm{C}-\mathrm{F}} 13.5,6.3\right), 118.5\left(\mathrm{dd},{ }^{1} J_{\mathrm{C}-\mathrm{F}} 264.5,244.6\right), 144.8$, $149.0\left(\mathrm{dd},{ }^{2} J_{\mathrm{C}-\mathrm{F}} 20.8,18.1\right)$ and $166.4 ; \delta_{\mathrm{F}}\left(471 \mathrm{MHz}\right.$, benzene- $\left.d_{6}\right)$ -121.32 and -104.77 (each $\left.d,{ }^{2} J_{F-F} 242.9\right) ; m / z\left(E S^{+}\right) 517.3\left(\mathrm{M}^{+}+\right.$ $23,50 \%)$ and $355.1(100)$. 
(2S,6S)-3,3-Difluoro-2-hydroxy-6-[(2R,3R)-2,3-

dihydroxybutyl]-4-[(E)-methoxycarbonylmethylidene]-2-(2methylbut-3-en-2-yl)tetrahydro-4H-pyran (83). Nitromethane (0.064 mL, $1.189 \mathrm{mmol})$ was added to a suspension of magnesium bromide $(0.11 \mathrm{~g}, 0.59 \mathrm{mmol})$ in ether $(0.39 \mathrm{~mL})$ followed by the SEM-ether $82(21 \mathrm{mg}, 0.043 \mathrm{mmol})$ in ether $(0.4$ $\mathrm{mL})$ and the mixture was stirred at rt for $24 \mathrm{~h}$. Water $(10 \mathrm{~mL})$ and EtOAc $(10 \mathrm{~mL})$ were added and the aqueous phase was extracted with EtOAc $(3 \times 10 \mathrm{~mL})$. The organic extracts were washed with brine $(40 \mathrm{~mL})$, dried $\left(\mathrm{MgSO}_{4}\right)$ and concentrated under reduced pressure. Chromatography of the residue (1:99 to 4:96 methanol:DCM) gave the title compound $\mathbf{8 3}$ (11 mg, 0.03 $\mathrm{mmol}, 70 \%)$ as a colourless oil, $R_{\mathrm{f}}=0.29$ (4:96 methanol:DCM) (Found: [M-H]', 363.1635. $\mathrm{C}_{17} \mathrm{H}_{25} \mathrm{O}_{6} \mathrm{~F}_{2}$ requires $\mathrm{M}, 363.1619$ ); $v_{\max } / \mathrm{cm}^{-1} 3397,2979,2953,2926,1724,1674,1437,1370,1282$, 1204, 1177, 1119, 1066, 1032, 970, 920 and 882; $\delta_{\mathrm{H}}(400 \mathrm{MHz}$, benzene- $\left.d_{6}\right) 1.00\left(3 \mathrm{H}, \mathrm{d}, J 6.3,4^{\prime \prime}-\mathrm{H}_{3}\right), 1.31-1.48\left(2 \mathrm{H}, \mathrm{m}, \mathrm{1}^{\prime \prime}-\mathrm{H}_{2}\right)$, 1.39 and 1.45 (each $3 \mathrm{H}, \mathrm{s}, 1^{\prime}-\mathrm{H}_{3}$ or $\left.2^{\prime}-\mathrm{CH}_{3}\right), 2.29(1 \mathrm{H}, \mathrm{t}, J 13.1,5-$ H), $2.76\left(1 \mathrm{H}\right.$, br. s, OH), $3.29\left(3 \mathrm{H}, \mathrm{s}, \mathrm{OCH}_{3}\right), 3.29\left(1 \mathrm{H}, \mathrm{m}, 3^{\prime \prime}-\mathrm{H}\right)$, $3.58\left(1 \mathrm{H}, \mathrm{m}, 2^{\prime \prime}-\mathrm{H}\right), 4.14\left(1 \mathrm{H}, \mathrm{dt}, J \mathrm{~J}\right.$, $\left.4.1,2.0,5-\mathrm{H}^{\prime}\right), 4.32-4.43(2$ $\mathrm{H}, \mathrm{m}, 6-\mathrm{H}, \mathrm{OH}), 5.04-5.14\left(2 \mathrm{H}, \mathrm{m}, 4^{\prime}-\mathrm{H}_{2}\right), 6.36(1 \mathrm{H}, \mathrm{dd}, J 17.7$, $\left.10.8,3^{\prime}-\mathrm{H}\right)$ and $6.50(1 \mathrm{H}, \mathrm{dd}, J 3.6,1.8,4-\mathrm{CH}) ; \delta_{\mathrm{C}}(125 \mathrm{MHz}$, benzene- $\left.d_{6}\right)$ 19.9, 22.9(m), 23.9(m), 34.3, 39.4, 46.4, 51.5, 67.2, 71.6, 73.0, 99.5 (dd, $\left.{ }^{2} J_{C-F} 30.7,24.4\right), 113.9,117.6$ (dd, ${ }^{3} J_{C-F} 13.5$, 6.3), $118.4\left(\mathrm{dd},{ }^{1} J_{\mathrm{C}-\mathrm{F}} 264.5,244.6\right), 144.6,148.8\left(\mathrm{dd},{ }^{2} J_{\mathrm{C}-\mathrm{F}} 20.8\right.$, 18.1) and $166.7 ; \delta_{F}\left(377 \mathrm{MHz}\right.$, benzene- $\left.d_{6}\right)-121.53$ and -104.94 (each d, $\left.{ }^{2} J_{F-F} 242.9\right) ; m / z\left(E S^{-}\right) 401.2\left([M+37]^{-}, 30 \%\right)$ and 399.1 $\left([\mathrm{M}+35]^{-}, 100\right)$ and $363.2\left([\mathrm{M}-\mathrm{H}]^{-}, 80\right)$.

(2S,6S)-3,3-Difluoro-2-hydroxy-6-[(2R,3R)-2-butanoyloxy-3hydroxybutyl]- and (2S,6S)-3,3-difluoro-2-hydroxy-6-[(2R,3R)3-butanoyloxy-2-hydroxybutyl]-4-[(E)-

methoxycarbonylmethylidene)-2-(2-methylbut-3-en-2-

yl)tetrahydro-4H-pyrans (84) and (86). Hydrogen fluoridepyridine complex $(0.12 \mathrm{~mL})$ was added to the bis-silyl ether 80 (25 mg, $0.038 \mathrm{mmol}$ ) and pyridine $(0.2 \mathrm{~mL})$ in THF $(1.9 \mathrm{~mL})$ at 0 ${ }^{\circ} \mathrm{C}$ and the mixture stirred for $16 \mathrm{~h}$. Saturated aqueous sodium hydrogen carbonate $(10 \mathrm{~mL})$ was added and the mixture was stirred for $1 \mathrm{~h}$. Ethyl acetate $(10 \mathrm{~mL})$ was added and the aqueous phase was extracted with EtOAc $(3 \times 10 \mathrm{~mL})$. The organic extracts were washed with brine $(40 \mathrm{~mL})$, dried $\left(\mathrm{MgSO}_{4}\right)$ and concentrated under reduced pressure. Chromatography of the residue (40:60 ether:light petroleum) gave the title compound $84(7 \mathrm{mg}, 0.016 \mathrm{mmol}, 42 \%)$ as a colourless oil containing $c a .10 \%$ of the regioisomer $86, R_{\mathrm{f}}=0.41(4: 96$ methanol:DCM) (Found: $\mathrm{M}^{+}+\mathrm{Na}, 457.2015 . \mathrm{C}_{21} \mathrm{H}_{32} \mathrm{O}_{7} \mathrm{~F}_{2} \mathrm{Na}$ requires $M, 457.2014) ; v_{\max } / \mathrm{cm}^{-1} 3393,2972,2934,2879,1724$, 1674, 1436, 1368, 1280, 1237, 1178, 1065, 1034, 924 and 882; $\delta_{\mathrm{H}}\left(500 \mathrm{MHz}\right.$, benzene- $\left.d_{6}\right) 0.71\left(3 \mathrm{H}, \mathrm{t}, J 7.3, \mathrm{CH}_{2} \mathrm{CH}_{3}\right), 0.96(3 \mathrm{H}$, d, J 6.5, 4"'- $\left.\mathrm{H}_{3}\right), 1.37-1.45\left(5 \mathrm{H}, \mathrm{m}, 1^{\prime \prime}-\mathrm{H}_{2}, 1^{\prime}-\mathrm{H}_{3}\right), 1.49\left(3 \mathrm{H}, \mathrm{s}, \mathrm{2}^{\prime}-\right.$ $\left.\mathrm{CH}_{3}\right), 1.60-1.63\left(2 \mathrm{H}, \mathrm{m}, \mathrm{CH}_{2} \mathrm{CH}_{3}\right), 1.94[2 \mathrm{H}, \mathrm{td}, J$ 7.7, 4.3, $\mathrm{C}(\mathrm{O}) \mathrm{CH}_{2}$ ], $2.29(1 \mathrm{H}, \mathrm{t}, \mathrm{J} 13.1,5-\mathrm{H}), 3.21\left(3 \mathrm{H}, \mathrm{s}, \mathrm{OCH}_{3}\right), 3.28(1 \mathrm{H}$, $\mathrm{s}, \mathrm{OH}), 3.50\left(1 \mathrm{H}, \mathrm{m}, 3^{\prime \prime}-\mathrm{H}\right), 3.56(1 \mathrm{H}, \mathrm{m}, \mathrm{OH}), 4.10(1 \mathrm{H}, \mathrm{t}, J 10.4$, 6-H), $4.19\left(1 \mathrm{H}, \mathrm{dt}, J\right.$ 14.1, 2.2, 5- $\left.\mathrm{H}^{\prime}\right), 5.09(1 \mathrm{H}, \mathrm{dd}, J$ 17.7, 1.2, 4'$H), 5.14\left(1 \mathrm{H}, \mathrm{m}, 2^{\prime \prime}-\mathrm{H}\right), 5.19\left(1 \mathrm{H}, \mathrm{dd}, J \mathrm{~J}\right.$, $\left.7,1.2,4^{\prime}-\mathrm{H}^{\prime}\right)$ and 6.44$6.50\left(2 \mathrm{H}, \mathrm{m}, 4-\mathrm{CH}, 3^{\prime}-\mathrm{H}\right)$; $\delta_{\mathrm{C}}\left(125 \mathrm{MHz}\right.$, benzene- $\left.d_{6}\right)$ 14.0, 19.1,
19.7, 22.3(m), 24.0(m), 34.2, 36.5, 37.0, $46.6\left(\mathrm{t},{ }^{3} J_{\mathrm{C}-\mathrm{F}} 2.7\right), 51.4$, $66.7,69.3,74.5,99.7\left(\mathrm{dd},{ }^{2} J_{C-F} 31.6,24.4\right), 114.7,117.8\left(\mathrm{dd},{ }^{3} J_{C-F}\right.$ $13.5,6.3), 118.3\left(\mathrm{dd},{ }^{1} J_{\mathrm{C}-\mathrm{F}} 264.5,242.8\right), 144.7,148.7$ (dd, ${ }^{2} J_{C-F}$ $21.7,19.0), 166.4$ and $174.3 ; \delta_{F}\left(377 \mathrm{MHz}\right.$, benzene- $\left.d_{6}\right)-120.93$ and -105.01 (each d, $\left.{ }^{2} J_{\mathrm{F}-\mathrm{F}} 244.2\right) ; \mathrm{m} / \mathrm{z}\left(\mathrm{ES}^{+}\right) 457.4\left(\mathrm{M}^{+}+23,80 \%\right)$ and $417.4\left(\mathrm{M}^{+}-17,100\right)$. Further elution gave the title compound $86(7 \mathrm{mg}, 0.016 \mathrm{mmol}, 42 \%)$ as a colourless oil, $R_{\mathrm{f}}=$ 0.35 (4:96 methanol:DCM) (Found: $\mathrm{M}^{+}+\mathrm{Na}, 457.2015$. $\mathrm{C}_{21} \mathrm{H}_{32} \mathrm{O}_{7} \mathrm{~F}_{2} \mathrm{Na}$ requires $\left.\mathrm{M}, 457.2014\right) ; v_{\max } / \mathrm{cm}^{-1} 3435,2955$, $2935,2878,1724,1674,1457,1436,1369,1281,1245,1198$, $1177,1117,1064,1010,920,881$ and $823 ; \delta_{\mathrm{H}}(400 \mathrm{MHz}$, benzene- $\left.d_{6}\right) 0.80\left(3 \mathrm{H}, \mathrm{t}, J 7.4, \mathrm{CH}_{2} \mathrm{CH}_{3}\right), 0.95(1 \mathrm{H}, \mathrm{br} . \mathrm{s}, \mathrm{OH})$, $1.07\left(3 \mathrm{H}, \mathrm{d}, \mathrm{J} 6.6,4^{\prime \prime}-\mathrm{H}_{3}\right), 1.25-1.43\left(8 \mathrm{H}, \mathrm{m}, 1^{\prime \prime}-\mathrm{H}_{2}, 1^{\prime}-\mathrm{H}_{3}, 2^{\prime}-\mathrm{CH}_{3}\right)$, $1.54\left(2 \mathrm{H}\right.$, sext, J 7.6, $\left.\mathrm{CH}_{2} \mathrm{CH}_{3}\right), 2.06\left[2 \mathrm{H}, \mathrm{td}, J 7.6,3.0, \mathrm{C}(\mathrm{O}) \mathrm{CH}_{2}\right]$, $2.28(1 \mathrm{H}, \mathrm{t}, J \mathrm{13.7}, 5-\mathrm{H}), 3.27\left(3 \mathrm{H}, \mathrm{s}, \mathrm{OCH}_{3}\right), 3.56(1 \mathrm{H}, \mathrm{d}, J$ 3.2, $\mathrm{OH}), 3.80$ (1 H, dd, J 7.1, 3.9, 2"'-H), 4.19 (1 H, dt, J 14.2, 2.4, 5$\left.H^{\prime}\right), 4.39(1 \mathrm{H}, \mathrm{t}, J 10.5,6-\mathrm{H}), 4.85(1 \mathrm{H}$, pent, J 6.1, 3"'-H), 4.97$5.05\left(2 \mathrm{H}, \mathrm{m}, 4^{\prime}-\mathrm{H}_{2}\right), 6.23\left(1 \mathrm{H}, \mathrm{dd}, J \mathrm{~J} .6,10.7,3^{\prime}-\mathrm{H}\right)$ and $6.50(1$ $\mathrm{H}, \mathrm{dd}, J 3.7,2.0,4-\mathrm{CH}) ; \delta_{\mathrm{C}}\left(125 \mathrm{MHz}\right.$, benzene- $\left.d_{6}\right) 14.0,16.3$, 19.1, 22.7, 23.9(m), 34.3, 36.7, 38.8, 46.5, 51.5, 67.1, 70.5, 73.6, $99.3\left(\mathrm{dd},{ }^{2} J_{\mathrm{C}-\mathrm{F}} 30.7,24.4\right), 114.3,117.6\left(\mathrm{dd},{ }^{3} J_{\mathrm{C}-\mathrm{F}} 12.6,5.4\right), 118.4$ (dd, $\left.{ }^{1} J_{C-F} 263.6,244.6\right), 144.5,148.6\left(d d,{ }^{2} J_{C-F} 21.7,19.0\right), 166.5$ and $173.4 ; \delta_{\mathrm{F}}\left(377 \mathrm{MHz}\right.$, benzene- $\left.d_{6}\right)-121.41\left(\mathrm{~d},{ }^{2} \mathrm{~J}_{\mathrm{F}-\mathrm{F}} 242.9\right)$ and $-105.19\left(\mathrm{~d},{ }^{2} J_{F-F} 241.5\right) ; m / z\left(E^{+}\right) 457.4\left(\mathrm{M}^{+}+23,100 \%\right), 452.4$ $\left(\mathrm{M}^{+}+18,60\right)$ and $417.3\left(\mathrm{M}^{+}-17,45\right)$.

(2S,6S)-3,3-Difluoro-2-hydroxy-6-[(2R,3R)-3-hydroxy-2-(2methylpropanoyloxy)butyl]- and (2S,6S)-3,3-difluoro-2hydroxy-6-[(2R,3R)-2-hydroxy-3-(2-methylpropanoyloxy)butyl]-4-[(E)-methoxycarbonylmethylidene)-2-(2-methylbut-3en-2-yl)tetrahydro-4H-pyrans (85) and (87). Following the procedure outlined for the synthesis of the esters $\mathbf{8 4}$ and $\mathbf{8 6}$, the hydrogen fluoride-pyridine complex $(0.32 \mathrm{~mL})$, the bis-silyl ether $81(67 \mathrm{mg}, 0.101 \mathrm{mmol})$ and pyridine $(0.5 \mathrm{~mL})$ in dry THF (5.1 $\mathrm{mL})$, after chromatography (70:30 ether:light petroleum) gave the title compound 85 (10 $\mathrm{mg}, 0.023 \mathrm{mmol}, 23 \%)$ as a colourless oil, $R_{f}=0.34$ (70:30 ether:light petroleum); $[\alpha]_{D}{ }^{20}=-54$ (c 0.50, ether) (Found: $\mathrm{M}^{+}+\mathrm{Na}, 457.1993 . \mathrm{C}_{21} \mathrm{H}_{32} \mathrm{O}_{7} \mathrm{~F}_{2} \mathrm{Na}$ requires $\mathrm{M}$, $457.2014) ; v_{\max } / \mathrm{cm}^{-1} 3480,2977,1724,1674,1436,1369,1280$, $1200,1067,1035$ and $923 ; \delta_{H}\left(400 \mathrm{MHz}\right.$, benzene- $\left.d_{6}\right) 0.94(\mathrm{~d}, J$ $\left.6.5,4^{\prime \prime}-\mathrm{H}_{3}\right), 0.94$ and 0.96 (each $\left.3 \mathrm{H}, \mathrm{d}, J 6.9, \mathrm{CHCH}_{3}\right), 1.41(3 \mathrm{H}$, d, J 4.0, 1'- $\left.\mathrm{H}_{3}\right), 1.49\left(3 \mathrm{H}, \mathrm{s}, 2^{\prime}-\mathrm{CH}_{3}\right), 1.58-1.64\left(2 \mathrm{H}, \mathrm{m}, 1^{\prime \prime}-\mathrm{H}_{2}\right)$, 2.19-2.33 [2 H, m, C(O)CH, 5-H], $3.19(1 \mathrm{H}, \mathrm{d}, J 3.9, \mathrm{OH}), 3.20$ (3 $\left.\mathrm{H}, \mathrm{s}, \mathrm{OCH}_{3}\right), 3.47\left(1 \mathrm{H}, \mathrm{m}, 3^{\prime \prime}-\mathrm{H}\right), 4.08(1 \mathrm{H}, \mathrm{m}, 6-\mathrm{H}), 4.18(1 \mathrm{H}, \mathrm{dt}$, J 14.0, 2.4, 5- $\left.\mathrm{H}^{\prime}\right), 5.07\left(1 \mathrm{H}, \mathrm{dd}, J 17.6,1.4,4^{\prime}-\mathrm{H}\right), 5.13(1 \mathrm{H}, \mathrm{m}$, $\left.2^{\prime \prime}-\mathrm{H}\right), 5.19\left(1 \mathrm{H}, \mathrm{dd}, J \mathrm{~J}\right.$. $\left.5,1.3,4^{\prime}-\mathrm{H}^{\prime}\right)$ and 6.42-6.51 (2 H, m, 4$\left.\mathrm{CH}, 3^{\prime}-\mathrm{H}\right) ; \delta_{\mathrm{C}}\left(101 \mathrm{MHz}\right.$, benzene- $\left.d_{6}\right) 19.1,19.2,19.4,19.7$, 21.9(m), 23.7 (m), 33.9(m), 34,3, 36.5, $46.3\left(\mathrm{t},{ }^{3} J_{\mathrm{C}-\mathrm{F}} 2.5\right), 51.0$, $66.3,69.0,73.8,98.8\left(\mathrm{dd},{ }^{2} J_{\mathrm{C}-\mathrm{F}} 31.8,24.6\right), 114.5,117.4\left(\mathrm{dd},{ }^{3} J_{\mathrm{C}-\mathrm{F}}\right.$ $13.2,6.1), 118.0\left(\mathrm{dd},{ }^{1} J_{\mathrm{C}-\mathrm{F}} 267.1,245.7\right), 144.4,148.3\left(\mathrm{dd},{ }^{2} J_{C-F}\right.$ $21.4,18.8), 166.0$ and $177.4 ; \delta_{F}\left(376 \mathrm{MHz}\right.$, benzene- $\left.d_{6}\right)-120.89$ and -104.98 (each d, $\left.{ }^{2} J_{F-F} 243.6\right) ; m / z\left(E^{+}\right) 457.2\left(\mathrm{M}^{+}+23,10 \%\right)$ and 301.1 (100). Further elution gave the title compound 87 (10 $\mathrm{mg}, 0.023 \mathrm{mmol}, 23 \%)$ as a white solid, $R_{\mathrm{f}}=0.17$ (70:30 ether:light petroleum), m.p. $81^{\circ} \mathrm{C},[\alpha]_{D}^{20}=-49.3$ (c 0.85, ether) (Found: $\mathrm{M}^{+}+\mathrm{Na}, 457.1993 . \mathrm{C}_{21} \mathrm{H}_{32} \mathrm{O}_{7} \mathrm{~F}_{2} \mathrm{Na}$ requires $\mathrm{M}, 457.2014$ ); $v_{\max } / \mathrm{cm}^{-1} 3435,2979,1725,1674,1436,1370,1281,1245,1201$, 
$1178,1118,1065,1011,920$ and $882 ; \delta_{H}\left(400 \mathrm{MHz}\right.$, benzene- $\left.d_{6}\right)$ $1.05\left(3 \mathrm{H}, \mathrm{d}, J\right.$ J 7.0, $\left.\mathrm{CHCH}_{3}\right), 1.06\left(3 \mathrm{H}, \mathrm{d}, J\right.$ 6.4, 4"'- $\left.\mathrm{H}_{3}\right), 1.07(3 \mathrm{H}, \mathrm{d}$ J 7.0, $\left.\mathrm{CHCH}_{3}\right), 1.30-1.39\left(4 \mathrm{H}, \mathrm{m}, 1^{\prime \prime}-\mathrm{H}, 1^{\prime}-\mathrm{H}_{3}\right), 1.41\left(3 \mathrm{H}, \mathrm{s}, 2^{\prime}-\mathrm{CH}_{3}\right)$ $1.50\left(1 \mathrm{H}, \mathrm{ddd}, J 14.2,10.2,2.2,1^{\prime \prime}-\mathrm{H}^{\prime}\right), 2.27(1 \mathrm{H}, \mathrm{m}, 5-\mathrm{H}), 2.37$ [1 H, hept, J 7.0, $\mathrm{C}(\mathrm{O}) \mathrm{CH}$ ], $2.67(1 \mathrm{H}$ br. s, OH), $3.29(3 \mathrm{H}, \mathrm{s}$, $\left.\mathrm{OCH}_{3}\right), 3.79-3.88\left(2 \mathrm{H}, \mathrm{m}, 2^{\prime \prime}-\mathrm{H}, \mathrm{OH}\right), 4.16(1 \mathrm{H}, \mathrm{dt}, \mathrm{J} 14.1,2.5,5-$ $\left.H^{\prime}\right), 4.38(1 \mathrm{H}, \mathrm{m}, 6-\mathrm{H}), 4.84\left(1 \mathrm{H}, \mathrm{m}, 3^{\prime \prime}-\mathrm{H}\right), 5.00-5.09\left(2 \mathrm{H}, \mathrm{m}, 4^{\prime}-\right.$ $\left.\mathrm{H}_{2}\right), 6.27\left(1 \mathrm{H}, \mathrm{dd}, J \mathrm{~J} .6,10.8,3^{\prime}-\mathrm{H}\right)$ and $6.48(1 \mathrm{H}, \mathrm{dd}, J 3.9,2.0$, $4-\mathrm{CH}) ; \delta_{\mathrm{C}}\left(101 \mathrm{MHz}\right.$, benzene- $\left.d_{6}\right) 15.8,19.1,19.2,22.3(\mathrm{~m})$, 23.5(m), 33.9(m), 34.3, 38.4, 46.1, 51.1, 66.8, 70.2, 73.1, 98.9 (dd, $\left.{ }^{2} J_{C-F} 31.3,24.8\right), 114.1,117.3$ (dd, $\left.{ }^{3} J_{C-F} 13.1,6.1\right), 118.1$ (dd, $\left.{ }^{1} J_{C-F} 264.7,246.8\right), 144.1,148.2\left(d d,{ }^{2} J_{C-F} 21.4,18.7\right), 166.2$ and 176.5; $\delta_{\mathrm{F}}\left(376 \mathrm{MHz}\right.$, benzene- $d_{6}$ ) -121.36 and -105.02 (each $d$, $\left.{ }^{2} J_{F-F} 241.9\right) ; m / z\left(\mathrm{ES}^{+}\right) 457.2\left(\mathrm{M}^{+}+23,100 \%\right)$.

(3RS,7S,9R,10R)-7,10-Bis(tert-butyldimethylsilyloxy)-4,4difluoro-3-hydroxy-5-[(E)-methoxycarbonylmethylidene]-2,2dimethyl-9-(2-trimethylsilylethoxy)methoxyundecanal (90). Osmium tetroxide in tert-butanol $(2.5 \%, 0.37 \mathrm{~mL})$ and $N$ methylmorpholine- $N$-oxide ( $46 \mathrm{mg}, 0.40 \mathrm{mmol}$ ) were added to the diene 77 (0.19 g, $0.26 \mathrm{mmol})$ in tert-butanol-water (1:1, 2.63 $\mathrm{mL}$ ) and the mixture stirred at $\mathrm{rt}$ for $4 \mathrm{~h}$. Saturated aqueous sodium thiosulfate $(20 \mathrm{~mL})$ and EtOAc $(20 \mathrm{~mL})$ were added and the aqueous phase was extracted with EtOAc $(3 \times 20 \mathrm{~mL})$. The organic extracts were washed with brine $(80 \mathrm{~mL})$, dried $\left(\mathrm{MgSO}_{4}\right)$ and concentrated under reduced pressure to give the dihydroxylated intermediate (Found: $\mathrm{M}^{+}+\mathrm{H}, 759.4490$. $\mathrm{C}_{35} \mathrm{H}_{73} \mathrm{O}_{9} \mathrm{~F}_{2} \mathrm{Si}_{3}$ requires $\left.\mathrm{M}, 759.4525\right) ; \mathrm{m} / e\left(\mathrm{ES}^{+}\right) 781.6\left(\mathrm{M}^{+}+23\right.$, $100 \%)$. The residue was dissolved in DCM $(2.6 \mathrm{~mL})$ and sodium carbonate ( $84 \mathrm{mg}, 0.79 \mathrm{mmol}$ ) and lead(IV) acetate $(0.14 \mathrm{~g}, 0.32$ $\mathrm{mmol})$ were added. The reaction mixture was stirred at $\mathrm{rt}$ for 5 min, filtered through Celite ${ }^{\circledast}$ and concentrated under reduced pressure. Chromatography of the residue (10:90 to 20:80 ether:light petroleum) gave the title compound 90 ( $0.16 \mathrm{~g}, 0.22$ mmol, $82 \%)$ as a colourless oil, a 50:50 mixture of diastereoisomers $\left({ }^{19} \mathrm{~F}\right.$ NMR), $R_{\mathrm{f}}=0.48 \quad(40: 60$ ether:light petroleum), $[\alpha]_{D}^{27}=-18.8$ (c 2.0, ether) (Found: $\mathrm{M}^{+}+\mathrm{Na}$, 749.4049. $\mathrm{C}_{34} \mathrm{H}_{68} \mathrm{O}_{8} \mathrm{~F}_{2} \mathrm{Si}_{3} \mathrm{Na}$ requires $\left.\mathrm{M}, 749.4082\right) ; v_{\max } / \mathrm{cm}^{-1}$ 2953, 2929, 2857, 1728, 1472, 1362, 1250, 1180, 1101, 1055, 1031 and 832; $\delta_{\mathrm{H}}\left(400 \mathrm{MHz}, \mathrm{CDCl}_{3}\right) 0.05\left(9 \mathrm{H}, \mathrm{s}, 3 \times \mathrm{SiCH}_{3}\right), 0.05$, $0.06,0.07$ and 0.08 (each $\left.3 \mathrm{H}, \mathrm{s}, \mathrm{SiCH}_{3}\right), 0.87(2)$ and $0.88(2)$ [each $4.5 \mathrm{H}, \mathrm{s}, \mathrm{SiC}\left(\mathrm{CH}_{3}\right)_{3}$ ], 0.91-0.99 $\left(2 \mathrm{H}, \mathrm{m}, \mathrm{SiCH}_{2}\right), 1.02$ and 1.04 (each $\left.1.5 \mathrm{H}, \mathrm{d}, J 6.3,11-\mathrm{H}_{3}\right), 1.22\left(3 \mathrm{H}, \mathrm{s}, 2-\mathrm{CH}_{3}\right), 1.24$ and 1.26 (each $\left.1.5 \mathrm{H}, \mathrm{s}, 2-\mathrm{CH}_{3}\right), 1.51(1 \mathrm{H}, \mathrm{m}, 8-\mathrm{H}), 1.77(0.5 \mathrm{H}, \mathrm{ddd}, J$ 14.6, 8.1, 1.3, 8- $\left.\mathrm{H}^{\prime}\right), 1.86\left(0.5 \mathrm{H}\right.$, ddd, J 14.4, 6.0, 1.5, 8- $\left.\mathrm{H}^{\prime}\right), 2.65$ (0.5 H, dd, J 13.4, 6.3, 6-H), $2.80(0.5 \mathrm{H}, \mathrm{dd}, J$ 13.6, 8.0, 6-H), 3.17 $\left(0.5 \mathrm{H}, \mathrm{dd}, J 14.4,6.6,6-\mathrm{H}^{\prime}\right), 3.30\left(0.5 \mathrm{H}, \mathrm{dd}, J 13.1,8.8,6-\mathrm{H}^{\prime}\right)$, $3.46\left(1 \mathrm{H}, \mathrm{m}, \mathrm{OHCHCH}_{2} \mathrm{Si}\right), 3.53(1 \mathrm{H}, \mathrm{m}, 9-\mathrm{H}), 3.66(1 \mathrm{H}, \mathrm{m}$, $\mathrm{OHCHCH}_{2} \mathrm{Si}$ ), 3.73 and 3.74 (each $\left.1.5 \mathrm{H}, \mathrm{s}, \mathrm{OCH}_{3}\right), 3.93(1.5 \mathrm{H}, \mathrm{m}$, 3- $\mathrm{H}, \mathrm{OH}), 4.00-4.10(1.5 \mathrm{H}, \mathrm{m}, 7-\mathrm{H}, 10-\mathrm{H}), 4.25(0.5 \mathrm{H}, \mathrm{m}, 7-\mathrm{H})$, 4.72, 4.73, 4.74 and 4.76 (each $0.5 \mathrm{H}, \mathrm{d}, \mathrm{J} 6.8, \mathrm{OHCHO}$ ), 6.19 (0.5 $\mathrm{H}, \mathrm{s}, 5-\mathrm{CH}), 6.25(0.5 \mathrm{H}, \mathrm{d}, J 2.8,5-\mathrm{CH})$ and 9.55 and 9.56 (each $0.5 \mathrm{H}, \mathrm{s}, 1-\mathrm{H}) ; \delta_{\mathrm{C}}\left(100 \mathrm{MHz}, \mathrm{CDCl}_{3}\right)-4.8,-4.7(2),-4.6,-4.1$, $-4.0,-1.5,17.0,17.1,18.0,18.1,18.7\left(\mathrm{t},{ }^{4} \mathrm{~J}_{\mathrm{C}-\mathrm{F}} 2.0\right), 18.8\left(\mathrm{t},{ }^{4} \mathrm{~J}_{\mathrm{C}-\mathrm{F}}\right.$ 2.9), 19.7(m), 19.8(m), 25.8, 25.9(2), 35.3, 35.4, 35.7, 36.5, $49.4(2), 51.5(2), 65.4(2), 68.7,68.8,69.0,70.8,74.4\left(\mathrm{dd},{ }^{2} J_{\mathrm{C}-\mathrm{F}}\right.$ 33.0, 25.6), $76.0\left(\mathrm{dd},{ }^{2} J_{\text {C-F }} 33.7,24.9\right), 80.6,80.9,96.0,121.7(\mathrm{~m})$,
121.7 and 122.8 (each dd, $\left.{ }^{1} J_{C-F} 257.9,245.4\right), 123.3$ (dd, ${ }^{3} J_{C-F}$ $12.5,6.6), 148.4\left(\mathrm{dd},{ }^{2} J_{\mathrm{C}-\mathrm{F}} 24.2,21.3\right), 151.0$ (dd, ${ }^{2} J_{\mathrm{C}-\mathrm{F}} 25.7,20.5$ ), 165.7, 165.8, 204.1 $(\mathrm{m})$ and 204.3; $\delta_{\mathrm{F}}\left(377 \mathrm{MHz}, \mathrm{CDCl}_{3}\right)-113.49$, $-111.92,-99.55$ and -98.94 (each $0.5 \mathrm{~F}, \mathrm{~d},{ }^{2} J_{\mathrm{F}-\mathrm{F}} 246.9$ ); $\mathrm{m} / \mathrm{z}\left(\mathrm{ES}^{+}\right.$) $749.6\left(\mathrm{M}^{+}+23,100 \%\right)$ and $609.4(60)$.

Methyl $(5 S, 7 R, 8 R, 2 E)-5,8-b i s(t e r t-b u t y l d i m e t h y l s i l y l o x y)-3-$ [(2RS)-1,1-difluoro-2,4-dihydroxy-3,3-dimethylbutyl]-7-(2trimethylsilylethoxy)methoxynon-2-enoate (91). Sodium borohydride $(9 \mathrm{mg}, 0.23 \mathrm{mmol}$ ) was added to the aldehyde 90 $(0.14 \mathrm{~g}, 0.19 \mathrm{mmol})$ in $\mathrm{MeOH}(2.0 \mathrm{~mL})$ at $0{ }^{\circ} \mathrm{C}$ and the mixture stirred for $1 \mathrm{~h}$. Aqueous hydrogen chloride $(10 \%, 10 \mathrm{~mL})$ was added at $0{ }^{\circ} \mathrm{C}$ followed by EtOAc $(10 \mathrm{~mL})$. The aqueous phase was extracted with EtOAc $(3 \times 10 \mathrm{~mL})$ and the organic extracts were washed with brine $(40 \mathrm{~mL})$, dried $\left(\mathrm{MgSO}_{4}\right)$ and concentrated under reduced pressure. Chromatography of the residue (10:90 to $30: 70$ ether:light petroleum) gave the title compound 91 ( $0.12 \mathrm{~g}, 0.16 \mathrm{mmol}, 83 \%)$ as a colourless oil, a 50:50 mixture of diastereoisomers ( $\left.{ }^{19} \mathrm{~F} \mathrm{NMR}\right), R_{\mathrm{f}}=0.18(40: 60$ ether:light petroleum), $[\alpha]_{\mathrm{D}}{ }^{28}=-11.7$ (c 1.8, ether) (Found: $\mathrm{M}^{+}+$ $\mathrm{H}$, 729.4387. $\mathrm{C}_{34} \mathrm{H}_{71} \mathrm{O}_{8} \mathrm{~F}_{2} \mathrm{Si}_{3}$ requires $\left.\mathrm{M}, 729.4419\right) ; v_{\max } / \mathrm{cm}^{-1}$ $3415,2953,2929,2886,2857,1730,1472,1463,1250,1179$, $1151,1102,1055,1033$ and $834 ; \delta_{\mathrm{H}}\left(400 \mathrm{MHz} \mathrm{CDCl}_{3}\right) 0.01(9 \mathrm{H}$, $\left.\mathrm{s}, 3 \times \mathrm{SiCH}_{3}\right), 0.05,0.06,0.08$ and 0.09 (each $\left.3 \mathrm{H}, \mathrm{s}, \mathrm{SiCH}_{3}\right), 0.88$ and 0.89 [each $9 \mathrm{H}, \mathrm{s}, \mathrm{SiC}\left(\mathrm{CH}_{3}\right)_{3}$ ], 0.92-1.00 $\left(2 \mathrm{H}, \mathrm{m}, \mathrm{SiCH}_{2}\right), 1.02-$ $1.09\left(6 \mathrm{H}, \mathrm{m}, 3^{\prime}-\mathrm{CH}_{3}, 9-\mathrm{H}_{3}\right), 1.12$ and 1.14 (each $1.5 \mathrm{H}, \mathrm{d}, \mathrm{J}$ 2.6, 3'$\left.\mathrm{CH}_{3}\right), 1.51$ and 1.78 (each $\left.1 \mathrm{H}, \mathrm{m}, 6-\mathrm{H}\right), 2.72(0.5 \mathrm{H}, \mathrm{dd}, J$ 13.1, 6.0, 4-H), $2.86(0.5 \mathrm{H}, \mathrm{dd}, J 13.4,7.3,4-\mathrm{H}), 3.12(0.5 \mathrm{H}, \mathrm{dd}, J$ 13.4, $\left.6.8,4-\mathrm{H}^{\prime}\right), 3.26\left(0.5 \mathrm{H}, \mathrm{dd}, J 13.1,8.6,4-\mathrm{H}^{\prime}\right), 3.35$ and 3.45 (each $\left.1 \mathrm{H}, \mathrm{d}, \mathrm{J} 10.8,4^{\prime}-\mathrm{H}\right), 3.47-3.56\left(2 \mathrm{H}, \mathrm{m}, 2^{\prime}-\mathrm{H}, \mathrm{OHCHCH}_{2} \mathrm{Si}\right), 3.67$ (1 $\left.\mathrm{H}, \mathrm{m}, \mathrm{OHCHCH}_{2} \mathrm{Si}\right), 3.73\left(3 \mathrm{H}, \mathrm{s}, \mathrm{OCH}_{3}\right), 3.74(1 \mathrm{H}, \mathrm{m}, 5-\mathrm{H}), 3.81$ $(0.5 \mathrm{H}, \mathrm{d}, \mathrm{J} 3.8, \mathrm{OH}), 3.87(0.5 \mathrm{H}, \mathrm{d}, J 3.5, \mathrm{OH}), 3.99-4.04(1 \mathrm{H}, \mathrm{m}$, 8-H), 4.10 and 4.19 (each $0.5 \mathrm{H}, \mathrm{m}, 7-\mathrm{H}$ ), 4.71 and 4.73 (each 0.5 $\mathrm{H}, \mathrm{d}, \mathrm{J} 6.8, \mathrm{OHCHO}), 4.73\left(1 \mathrm{H}, \mathrm{s}, \mathrm{OCH}_{2} \mathrm{O}\right), 6.20(0.5 \mathrm{H}, \mathrm{s}, 2-\mathrm{H})$ and $6.26(0.5 \mathrm{H}, \mathrm{d}, J 2.6,2-\mathrm{H}) ; \delta_{\mathrm{C}}\left(100 \mathrm{MHz}, \mathrm{CDCl}_{3}\right)-4.8,-4.7(2)$, $-4.6,-4.1,-4.0,-1.5(2), 17.2,17.3,18.0,18.1,20.1\left(\mathrm{t},{ }^{4} J_{\mathrm{C}-\mathrm{F}} 4.4\right)$, $20.5\left(\mathrm{t},{ }^{4} J_{\text {C-F }}\right.$ 3.7), $22.9\left(\mathrm{~d},{ }^{4} J_{\mathrm{C}-\mathrm{F}} 5.1\right), 23.5\left(\mathrm{~d},{ }^{4} J_{\mathrm{C}-\mathrm{F}} 4.4\right), 26.0(3)$, 35.6, 35.7, 36.2, 36.3, 38.9, 39.2, 51.4, 65.3, 65.8, 68.9(2), 69.1, $70.1,72.0\left(\mathrm{~d},{ }^{5} J_{\mathrm{C}-\mathrm{F}} 2.2\right), 72.4\left(\mathrm{~d},{ }^{5} J_{\mathrm{C}-\mathrm{F}} 2.2\right), 75.6\left(\mathrm{dd},{ }^{2} J_{\mathrm{C}-\mathrm{F}} 31.5\right.$, 25.6), $78.4\left(d d,{ }^{2} J_{C-F} 32.2,25.6\right), 80.4(2), 95.8,95.9,121.8\left(t,{ }^{3} J_{C-F}\right.$ 9.5), 122.7 (dd, $\left.{ }^{3} J_{C-F} 11.7,6.6\right), 122.7$ and 123.4 (each dd, ${ }^{1} J_{C-F}$ 258.2, 245.8), 149.6 (dd, $\left.{ }^{2} J_{C-F} 23.5,21.3\right), 151.3$ (dd, ${ }^{2} J_{C-F} 24.9$, 20.5), 165.9 and 166.0; $\delta_{\mathrm{F}}\left(377 \mathrm{MHz}, \mathrm{CDCl}_{3}\right)-115.99,-114.44$, -99.57 and -99.30 (each $\left.0.5 \mathrm{~F}, \mathrm{~d},{ }^{2} J_{\mathrm{F}-\mathrm{F}} 244.2\right) ; \mathrm{m} / \mathrm{z}\left(\mathrm{ES}^{+}\right) 751.6$ $\left(\mathrm{M}^{+}+23,50 \%\right)$ and $611.5(100)$.

Methyl $(5 S, 7 R, 8 R, 2 E)-5,8-b i s(t e r t-b u t y l d i m e t h y l s i l y l o x y)-3-$ \{1,1-difluoro-3,3-dimethyl-4-[2-(2,2,2trichloroethoxycarbonylethoxy)benzoyloxy]-2-oxobutyl\}-7-(2trimethylsilylethoxymethoxy)non-2-enoate (93). 2,4,6Trichlorobenzoyl chloride $(0.018 \mathrm{~mL}, 0.11 \mathrm{mmol})$ and triethylamine $(0.034 \mathrm{~mL}, 0.24 \mathrm{mmol})$ were added to the carboxylic acid $98(38 \mathrm{mg}, 0.11 \mathrm{mmol})$ in toluene $(0.3 \mathrm{~mL})$ and the mixture stirred for $1.5 \mathrm{~h}$. The diol 91 (68 $\mathrm{mg}, 0.093 \mathrm{mmol}$ ) and DMAP (11 mg, $0.093 \mathrm{mmol}$ ) in toluene $(1.45 \mathrm{~mL}$ ) were added and the mixture stirred for $16 \mathrm{~h}$. Saturated aqueous ammonium chloride $(10 \mathrm{~mL})$ and ether $(10 \mathrm{~mL})$ were added and 
the aqueous phase was extracted with ether $(3 \times 10 \mathrm{~mL})$. The organic extracts were washed with brine $(40 \mathrm{~mL})$, dried $\left(\mathrm{MgSO}_{4}\right)$ and concentrated under reduced pressure to give the ester 92 $(0.10 \mathrm{~g})$ as a mixture of diastereoisomers used without further purification, $R_{\mathrm{f}}=0.38$ (40:60 ether:light petroleum).

This ester 92 was taken up in DCM $(1.6 \mathrm{~mL})$ and $4 \AA$ molecular sieves (75 mg), $\mathrm{N}$-methylmorpholine- $\mathrm{N}$-oxide $(22 \mathrm{mg}$, $0.17 \mathrm{mmol}$ ) and TPAP (5 mg, $0.013 \mathrm{mmol}$ ) were added. After stirring at $\mathrm{rt}$ for $16 \mathrm{~h}$, the reaction mixture was concentrated under reduced pressure. Chromatography of the residue that had been dry-loaded onto the column (5:95 to 10:90 ether:light petroleum) gave the title compound $93(56 \mathrm{mg}, 0.053 \mathrm{mmol}$, $57 \%$ from diol 91 ) as a colourless oil, $R_{\mathrm{f}}=0.18$ (20:80 ether:light petroleum), $[\alpha]_{D}^{29}=-11.4$ (c 3.6, ether); $v_{\max } / \mathrm{cm}^{-1} 2953,2929$, 2857, 1760, 1730, 1602, 1472, 1452, 1370, 1300, 1249, 1153, $1098,1076,1051,1032,933,833$ and $809 ; \delta_{\mathrm{H}}\left(400 \mathrm{MHz}, \mathrm{CDCl}_{3}\right)$ $0.01\left(9 \mathrm{H}, \mathrm{s}, 3 \times \mathrm{SiCH}_{3}\right), 0.03,0.04,0.05$ and 0.06 (each $3 \mathrm{H}, \mathrm{s}$, $\left.\mathrm{SiCH}_{3}\right), 0.85$ and 0.88 [each $9 \mathrm{H}, \mathrm{s}, \mathrm{SiC}\left(\mathrm{CH}_{3}\right)_{3}$ ], $0.90-0.99(2 \mathrm{H}, \mathrm{m}$, $\left.\mathrm{SiCH}_{2}\right), 1.03\left(3 \mathrm{H}, \mathrm{d}, \mathrm{J} 6.4,9-\mathrm{H}_{3}\right), 1.38\left(6 \mathrm{H}, \mathrm{s}, 2 \times 3^{\prime}-\mathrm{CH}_{3}\right), 1.42(1$ $\mathrm{H}, \mathrm{m}, 6-\mathrm{H}), 1.70\left(1 \mathrm{H}, \mathrm{dd}, \mathrm{J} 13.2,8.1,6-\mathrm{H}^{\prime}\right), 2.86-2.95(2 \mathrm{H}, \mathrm{m}, 4-$ $\left.\mathrm{H}_{2}\right), 3.03\left(2 \mathrm{H}, \mathrm{t}, J \mathrm{~J} .6, \mathrm{CH}_{2} \mathrm{CO}_{2} \mathrm{CH}_{2} \mathrm{CCl}_{3}\right), 3.46(1 \mathrm{H}, \mathrm{m}$, $\left.\mathrm{OHCHCH}_{2} \mathrm{Si}\right), 3.52(1 \mathrm{H}, \mathrm{m}, 7-\mathrm{H}), 3.63\left(3 \mathrm{H}, \mathrm{s}, \mathrm{OCH}_{3}\right), 3.68(1 \mathrm{H}$, ddd, J 11.8, 9.8, 5.4, $\left.\mathrm{OHCHCH}_{2} \mathrm{Si}\right), 3.98-4.10(2 \mathrm{H}, \mathrm{m}, 5-\mathrm{H}, 8-\mathrm{H})$, $4.37\left(2 \mathrm{H}, \mathrm{t}, J\right.$ J 6.4, $\left.\mathrm{OCH}_{2} \mathrm{CH}_{2} \mathrm{CO}_{2}\right), 4.41\left(2 \mathrm{H}, \mathrm{s}, 4^{\prime}-\mathrm{H}_{2}\right), 4.70(2 \mathrm{H}, \mathrm{s}$, $\left.\mathrm{OCH}_{2} \mathrm{O}\right), 4.80\left(2 \mathrm{H}, \mathrm{s}, \mathrm{CH}_{2} \mathrm{CCl}_{3}\right), 6.14(1 \mathrm{H}, \mathrm{s}, 2-\mathrm{H}), 6.97-7.01(2 \mathrm{H}$, $\mathrm{m}, \mathrm{ArH}), 7.47(1 \mathrm{H}, \mathrm{m}, \mathrm{ArH})$ and $7.68(1 \mathrm{H}, \mathrm{dd}, J \mathrm{7.6}, 1.2, \mathrm{ArH}) ; \delta_{\mathrm{C}}$ $\left(100 \mathrm{MHz}, \mathrm{CDCl}_{3}\right)-4.8,-4.7,-4.1,-1.5,17.1,17.9,18.1$, 21.9(m), 25.9(2), 34.0, 35.7, 36.0, 47.8, 51.5, 64.1, 65.1, 69.1(2), 69.7, 74.0, 80.2, 94.7, 96.0, 113.5, $116.9\left(\mathrm{t},{ }^{1} J_{\mathrm{C}-\mathrm{F}} 257.4\right), 119.9$, $120.8,124.0\left(\mathrm{t},{ }^{3} J_{\mathrm{C}-\mathrm{F}} 8.8\right), 125.5,131.7,133.7,146.3\left(\mathrm{t},{ }^{2} J_{\mathrm{C}-\mathrm{F}} 21.9\right)$, $158.1,165.0,165.2,169.3$ and $200.6\left(t,{ }^{2} J_{C-F} 29.9\right) ; \delta_{F}(377 \mathrm{MHz}$, $\mathrm{CDCl}_{3}$ ) -101.86 and -101.04 (each d, ${ }^{2} J_{\mathrm{F}-\mathrm{F}} 271.5$ ); $\mathrm{m} / \mathrm{z}\left(\mathrm{ES}^{+}\right) 1072$ $\left(\mathrm{M}^{+}+23,30 \%\right)$.

Methyl $\quad(5 S, 7 R, 8 R, 2 E)-5,8$-bis(tert-butyldimethylsilyloxy)-3\{1,1-difluoro-3,3-dimethyl-4-[2-(2,2,2trichloroethoxycarbonylethoxy)benzoyloxy]-2-oxobutyl\}-7hydroxynon-2-enoate (94). Nitromethane (0.14 mL, $2.52 \mathrm{mmol})$ was added to a suspension of magnesium bromide $(0.23 \mathrm{~g}, 1.26$ $\mathrm{mmol}$ ) in ether $(1.2 \mathrm{~mL})$ followed by the SEM-ether $93(95 \mathrm{mg}$, $0.09 \mathrm{mmol})$ in ether $(1.0 \mathrm{~mL})$. After stirring at $\mathrm{rt}$ for $15 \mathrm{~h}$, water $(10 \mathrm{~mL})$ and EtOAc $(10 \mathrm{~mL})$ were added and the aqueous phase was extracted with EtOAc $(3 \times 10 \mathrm{~mL})$. The organic extracts were washed with brine $(40 \mathrm{~mL})$, dried $\left(\mathrm{MgSO}_{4}\right)$ and concentrated under reduced pressure. Chromatography of the residue (10:90 ether:light petroleum) gave the title compound $94(54 \mathrm{mg}, 0.06$ $\mathrm{mmol}, 65 \%)$ as a colourless oil, $R_{\mathrm{f}}=0.33$ (40:60 ether:light petroleum), $[\alpha]_{D}^{30}=+0.50$ (c 2.0, ether); $v_{\max } / \mathrm{cm}^{-1} 2953,2929$, 2856, 1759, 1731, 1601, 1491, 1472, 1452, 1370, 1300, 1250, 1152, 1076 and 952; $\delta_{\mathrm{H}}\left(500 \mathrm{MHz}, \mathrm{CDCl}_{3}\right) 0.03,0.05,0.06$ and 0.09 (each $3 \mathrm{H}, \mathrm{s}, \mathrm{SiCH}_{3}$ ), 0.86 and 0.87 [each $9 \mathrm{H}, \mathrm{m}, \mathrm{SiC}\left(\mathrm{CH}_{3}\right)_{3}$ ], $1.13\left(3 \mathrm{H}, \mathrm{d}, \mathrm{J} 6.4,9-\mathrm{H}_{3}\right), 1.39\left(6 \mathrm{H}, \mathrm{s}, 2 \times 3^{\prime}-\mathrm{CH}_{3}\right), 1.45-1.53(2 \mathrm{H}$, m, 6- $\left.\mathrm{H}_{2}\right), 2.27(1 \mathrm{H}$, br. s, 7-OH), $2.87(1 \mathrm{H}, \mathrm{dd}, J 12.9,6.3,4-\mathrm{H})$, $2.99\left(1 \mathrm{H}, \mathrm{dd}, J \mathrm{12.3}, 8.2,4-\mathrm{H}^{\prime}\right), 3.02(2 \mathrm{H}, \mathrm{t}, J 6.3$, $\left.\mathrm{CH}_{2} \mathrm{CO}_{2} \mathrm{CH}_{2} \mathrm{CCl}_{3}\right), 3.49(1 \mathrm{H}, \mathrm{m}, 7-\mathrm{H}), 3.59(1 \mathrm{H}, \mathrm{m}, 8-\mathrm{H}), 3.65(3 \mathrm{H}$, $\left.\mathrm{s}, \mathrm{OCH}_{3}\right), 4.22(1 \mathrm{H}, \mathrm{m}, 5-\mathrm{H}), 4.38\left(2 \mathrm{H}, \mathrm{t}, \mathrm{J} 6.7, \mathrm{OCH}_{2} \mathrm{CH}_{2}\right), 4.42$ (2 $\left.\mathrm{H}, \mathrm{s}, 4^{\prime}-\mathrm{H}_{2}\right), 4.80\left(2 \mathrm{H}, \mathrm{s}, \mathrm{CH}_{2} \mathrm{CCl}_{3}\right), 6.14(1 \mathrm{H}, \mathrm{s}, 2-\mathrm{H}), 6.98-7.01(2$
$\mathrm{H}, \mathrm{m}, \mathrm{ArH}), 7.47(1 \mathrm{H}, \mathrm{m}, \mathrm{ArH})$ and $7.69(1 \mathrm{H}, \mathrm{dd}, \mathrm{J}$ 7.9, 1.9, ArH); $\delta_{\mathrm{C}}\left(100 \mathrm{MHz}, \mathrm{CDCl}_{3}\right)-5.0,-4.4,-4.3,17.9,20.1,21.9\left(\mathrm{~d},{ }^{4} J_{\mathrm{C}-\mathrm{F}} 2.2\right)$, 25.8(2), 34.0, 35.4, 40.6, 47.8, 51.6, 64.1, 68.5, 69.7, 71.7, 71.8, $74.0,94.7,113.5,116.9\left(\mathrm{t},{ }^{1} J_{\mathrm{C}-\mathrm{F}} 258.2\right), 119.9,120.8,123.8\left(\mathrm{t},{ }^{3} \mathrm{~J}_{\mathrm{C}-\mathrm{F}}\right.$ 9.5), 125.5, 131.7, 133.7, $146.8\left(t,{ }^{2} J_{C-F} 21.9\right), 158.1,164.9,165.2$, 169.3 and $200.5\left(\mathrm{t},{ }^{2} \mathrm{~J}_{\mathrm{C}-\mathrm{F}} 29.9\right) ; \delta_{\mathrm{F}}\left(471 \mathrm{MHz}, \mathrm{CDCl}_{3}\right)-102.07$ (d, $\left.{ }^{2} J_{F-F} 275.9\right)$ and $-100.55\left(d,{ }^{2} J_{F-F} 274.2\right) ; m / z\left(E S^{+}\right) 942\left(\mathrm{M}^{+}+23\right.$, $80 \%)$.

Zinc dust $(7 \mathrm{mg}$ ) and PPTS (14 mg, $0.055 \mathrm{mmol}$ ) were added to the ester $94(20 \mathrm{mg}, 0.022 \mathrm{mmol})$ in THF $(0.4 \mathrm{~mL})$ and the mixture heated under reflux for $30 \mathrm{~min}$. Saturated aqueous ammonium chloride $(10 \mathrm{~mL})$ was added and the mixture was extracted with EtOAc $(4 \times 10 \mathrm{~mL})$. The organic extracts were washed with brine $(40 \mathrm{~mL})$, dried $\left(\mathrm{MgSO}_{4}\right)$ and concentrated under reduced pressure to give the acid 95 . This acid was dissolved in benzene $(0.26 \mathrm{~mL})$. Pyridine $(5 \mu \mathrm{L}, 0.063 \mathrm{mmol})$ and 2,4,6-trichlorobenzoyl chloride ( $3 \mu \mathrm{L}, 0.016 \mathrm{mmol}$ ) were added and the mixture was stirred for $45 \mathrm{~min}$ at $\mathrm{rt}$ then added to DMAP (16 mg, $0.13 \mathrm{mmol}$ ) in benzene $(2.8 \mathrm{~mL}$ ). The mixture was heated at $60{ }^{\circ} \mathrm{C}$ for $16 \mathrm{~h}$ then cooled to rt. Saturated aqueous ammonium chloride $(10 \mathrm{~mL})$ was added and the mixture was extracted with EtOAc $(4 \times 10 \mathrm{~mL})$. The organic extracts were washed with brine $(40 \mathrm{~mL})$, dried $\left(\mathrm{MgSO}_{4}\right)$ and concentrated under reduced pressure to give an impure product provisionally identified as the acrylate $96(29 \mathrm{mg}) ; \delta_{\mathrm{H}}\left(400 \mathrm{MHz}, \mathrm{CDCl}_{3}\right) 0.05$ (12 $\mathrm{H}$, overlapping s, $\left.4 \times \mathrm{SiCH}_{3}\right), 1.15\left(3 \mathrm{H}, \mathrm{d}, J \mathrm{~J} .0,9-\mathrm{H}_{3}\right), 1.24[18$ $\left.\mathrm{H}, \mathrm{s}, 2 \times \mathrm{SiC}\left(\mathrm{CH}_{3}\right)_{3}\right], 1.33$ and 1.34 (each $\left.3 \mathrm{H}, \mathrm{s}, 3^{\prime}-\mathrm{CH}_{3}\right), 1.65(2 \mathrm{H}$, $\left.\mathrm{m}, 6-\mathrm{H}_{2}\right), 2.45\left(2 \mathrm{H}, \mathrm{m}, 4-\mathrm{H}_{2}\right), 3.47(1 \mathrm{H}, \mathrm{m}, 8-\mathrm{H}), 3.72(3 \mathrm{H}, \mathrm{s}$, $\left.\mathrm{OCH}_{3}\right), 3.85(1 \mathrm{H}, \mathrm{m}, 5-\mathrm{H}), 4.15(1 \mathrm{H}, \mathrm{d}, J$ 13.0, 4'-H), $4.27(1 \mathrm{H}, \mathrm{m}$, 7-H), $4.44\left(2 \mathrm{H}, \mathrm{m}, \mathrm{OH}, 4^{\prime}-\mathrm{H}^{\prime}\right), 6.19\left(1 \mathrm{H}, \mathrm{d}, J 11.0,3^{\prime \prime}-\mathrm{H}\right), 6.23(1$ $\mathrm{H}, \mathrm{m}, 2-\mathrm{H}), 6.40(1 \mathrm{H}, \mathrm{dd}, J$ 15.0, 11.0, 2"-H), $6.67(1 \mathrm{H}, \mathrm{d}, J$ 15.0, $\left.3^{\prime \prime}-\mathrm{H}^{\prime}\right), 7.16(1 \mathrm{H}, \mathrm{d}, J$ 6.0, ArH), $7.35(1 \mathrm{H}, \mathrm{t}, J$ 6.0, ArH), $7.61(1 \mathrm{H}$, $\mathrm{m}, \mathrm{ArH})$ and $7.96(1 \mathrm{H}, \mathrm{m}, \mathrm{ArH})$. When the macrocyclisation was attempted at $\mathrm{rt}$, the acrylate 96 was detected by TLC $10 \mathrm{~min}$ after adding the mixture to the DMAP.

2-(2,2,2-Trichloroethoxycarbonylethoxy)benzoic acid (98). (2,2,6,6-Tetramethylpiperidin-1yl)oxidanyl (TEMPO) (28 mg, $0.18 \mathrm{mmol})$ and $\mathrm{PhI}(\mathrm{OAc})_{2}(0.56 \mathrm{~g}, 1.728 \mathrm{mmol})$ were added to methyl 2-(3-hydroxypropoxy)benzoate $(0.125 \mathrm{~g}, 0.594 \mathrm{mmol})$ in MeCN $(12.0 \mathrm{~mL})$ and water $(1.9 \mathrm{~mL})$ and the mixture stirred at $\mathrm{rt}$ for $1 \mathrm{~h}$. 2-Methylbut-2-ene $(3.1 \mathrm{~mL}, 29.16 \mathrm{mmol})$, water $(0.96$ $\mathrm{mL}$ ) and $\mathrm{NaH}_{2} \mathrm{PO}_{4}(0.713 \mathrm{~g}, 5.94 \mathrm{mmol})$ were added. After cooling the mixture to $0{ }^{\circ} \mathrm{C}, \mathrm{NaClO}_{2}(0.43 \mathrm{~g}, 4.75 \mathrm{mmol})$ was added and the deep red solution was stirred for $1 \mathrm{~h}$. Saturated aqueous sodium thiosulfate $(20 \mathrm{~mL})$ and EtOAc $(20 \mathrm{~mL})$ were added and the aqueous phase was extracted into EtOAc $(3 \times 20$ $\mathrm{mL})$. The organic extracts were washed with brine $(80 \mathrm{~mL})$, dried $\left(\mathrm{MgSO}_{4}\right)$ and concentrated under reduced pressure. 2,2,2Trichloroethanol $(0.086 \mathrm{~mL}, 0.89 \mathrm{mmol})$, DCC $(0.185 \mathrm{~g}, 0.89$ mmol) and DMAP $(0.11 \mathrm{~g}, 0.89 \mathrm{mmol})$ were added to the residue in dry DCM $(3.1 \mathrm{~mL})$ and the mixture stirred at $\mathrm{rt}$ for 16 h. After concentration under reduced pressure, chromatography of the residue with dry-loading (2:98 to 5:95 EtOAc:light petroleum) gave the ester $97(0.16 \mathrm{~g}, 0.44 \mathrm{mmol}, 73 \%$ as a colourless oil, $R_{\mathrm{f}}=0.56$ (40:60 EtOAc:light petroleum) (Found: $\mathrm{M}^{+}+\mathrm{H}, 354.9900 . \mathrm{C}_{13} \mathrm{H}_{14} \mathrm{O}_{5}{ }^{35} \mathrm{Cl}_{3}$ requires 354.9907); $v_{\max } / \mathrm{cm}^{-1}$ 
2951, 1756, 1728, 1601, 1583, 1491, 1453, 1433, 1398, 1304, $1248,1153,1133,1083,1047,1026,912$ and 857; $\delta_{H}(400 \mathrm{MHz}$, $\left.\mathrm{CDCl}_{3}\right) 3.01\left(2 \mathrm{H}, \mathrm{t}, \mathrm{J}\right.$ 6.3, 1- $\left.\mathrm{H}_{2}\right), 3.85\left(3 \mathrm{H}, \mathrm{s}, \mathrm{OCH}_{3}\right), 4.38(2 \mathrm{H}, \mathrm{t}, \mathrm{J}$ 6.1, 2- $\left.\mathrm{H}_{2}\right), 4.79\left(2 \mathrm{H}, \mathrm{s}, \mathrm{CH}_{2} \mathrm{CCl}_{3}\right), 7.00(2 \mathrm{H}, \mathrm{t}, J \mathrm{~J} .8, \mathrm{ArH}), 7.45$ (1 $\mathrm{H}, \mathrm{dt}, J$ 7.3, 1.7, $\mathrm{ArH})$ and $7.77(1 \mathrm{H}, \mathrm{dd}, J$ 8.4, 2.0, $\mathrm{ArH}) ; \delta_{\mathrm{C}}(100$ $\mathrm{MHz}, \mathrm{CDCl}_{3}$ ) 34.2, 51.9, 64.2, 74.0, 94.6, 113.7, 120.7, 120.9, 131.6, 133.4, 157.7, 166.6 and 169.2; $\mathrm{m} / \mathrm{z}\left(\mathrm{ES}^{+}\right) 376.9\left(\mathrm{M}^{+}+23\right.$, $70 \%$ and $355.0\left(\mathrm{M}^{+}+1,100\right)$.

Sodium iodide $(0.265 \mathrm{~g}, 1.77 \mathrm{mmol})$ and trimethylsilyl chloride $(0.09 \mathrm{~mL}, 0.71 \mathrm{mmol})$ were added to this ester 97 (0.126 $\mathrm{g}, 0.354 \mathrm{mmol})$ in 1,2-dichloroethane $(3.6 \mathrm{~mL})$ and the solution heated at $100{ }^{\circ} \mathrm{C}$ for $15 \mathrm{~h}$ then cooled and concentrated under reduced pressure. Chromatography of the residue with dry-loading (5:95 to 20:80 EtOAc:light petroleum with $0.1 \%$ acetic acid) gave the title compound $98(90 \mathrm{mg}, 0.256 \mathrm{mmol}$, $72 \%)$ as a colourless oil, $R_{\mathrm{f}}=0.36$ (40:60 EtOAc:light petroleum) (Found: $\mathrm{M}^{+}+\mathrm{H}, 340.9749 . \mathrm{C}_{12} \mathrm{H}_{12} \mathrm{O}_{5}{ }^{35} \mathrm{Cl}_{3}$ requires $\mathrm{M}, 340.9750$ ); $\delta_{\mathrm{H}}\left(400 \mathrm{MHz}, \mathrm{CDCl}_{3}\right) 3.11\left(2 \mathrm{H}, \mathrm{t}, J\right.$ J 6.0, 1- $\left.\mathrm{H}_{2}\right), 4.57(2 \mathrm{H}, \mathrm{t}, \mathrm{J} 6.0,2-$ $\left.\mathrm{H}_{2}\right), 4.84\left(2 \mathrm{H}, \mathrm{s}, \mathrm{CH}_{2} \mathrm{CCl}_{3}\right), 7.08(1 \mathrm{H}, \mathrm{d}, J$ 8.3, $\mathrm{ArH}), 7.16(1 \mathrm{H}, \mathrm{t}, J$ 7.5, ArH), $7.58(1 \mathrm{H}, \mathrm{dt}, J$ 7.6, 1.8, ArH), $8.16(1 \mathrm{H}, \mathrm{dd}, J$ 7.8, 1.8, ArH) and $10.69\left(1 \mathrm{H}\right.$, br. s, $\left.\mathrm{CO}_{2} \mathrm{H}\right) ; \delta_{\mathrm{C}}\left(100 \mathrm{MHz} \mathrm{CDCl}_{3}\right) 33.8,64.7$, $74.2,94.4,112.6,118.2,122.6,133.9,135.0,156.9,165.7$ and 168.9; $m / z\left(\mathrm{ES}^{+}\right)$364.9, 362.9 (both $\left.\mathrm{M}^{+}+23,50 \%\right), 225.1$ (100).

\section{Acknowledgements}

We thank the EPSRC for support (C. E. R.).

\section{Notes and references}

1. (a) J. H. Sharp, M. K. Winson and J. S. Porter, Nat. Prod. Reports, 2007, 24, 659; (b) P. A. Wender, Products in Medicinal Chemistry, ed. S. Hanessian, Wiley-VCH Verlag GmbH \& Co., KgaA, Weinheim, Germany, 2014, 475; (c) H.-B. Yu, F. Yang, F. Sun, G.-Y. Ma, J.-H. Gan, W.-Z. Hu, B.-N. Han, W.-H. Jiao and H.-W. Lin, J. Nat Prod., 2014, 77, 2124.

2. (a) B.-F. Ruan and H.-L. Zhu, Curr. Med. Chem., 2012, 19, 2652; (b) K. Irie and R. C. Yanagita, Chem. Record, 2014, 14, 251.

3. (a) S. Ueno, R. C. Yanagita, K. Murakami, A. Murakami, H. Tokuda, N. Suzuki, T. Fujiwara and K. Irie, Biosci. Biotechnol. Biochem., 2012, 76, 1041; (b) D. E. Schaufelberger, M. P. Koleck, J. A. Beutler, A. M. Vatakis, A. B. Alvarado, P. Andrews, L. V. Marzo, G. M. Muschik, J. Roach, J. T. Ross et al., J. Nat. Prod., 1991, 54, 1265.

4. K. J. Hale and S. Manaviazar, Chem. Asian J., 2010, 5, 704.

5. (a) M. Kageyama, T. Tamura, M. H. Nantz, J. C. Roberts, P. Somfrai, D. C. Whritenour and S. Masamune, S. J. Am. Chem. Soc., 1990, 112, 7407; (b) D. A. Evans, P. H. Carter, E. M. Carreira, A. B. Charette, J. A. Prunet and M. Lautens, J. Am. Chem. Soc., 1999, 121, 7540; (c) K. Ohmori, Y. Ogawa, T. Obitsu, Y. Ishikawa, S Nishiyama and S. Yamamura, Angew. Chem. Int. Ed., 2000, 39, 2290; (d) S. Manaviazar, M. Frigerio, G. S. Bhatia, M. G. Hummersone, A. E. Aliev and K. J. Hale, Org. Lett., 2006, 8, 4477; (e) B. M. Trost and G. Dong, J. Am. Chem. Soc., 2010, 132, 16403; (f) G. E. Keck, Y. B. Poudel, T. J. Cummins, A. Rudra and J. A. Covel, J. Am.
Chem. Soc., 2011, 133, 744; (g) P. A. Wender and A. J. Schrier, J. Am. Chem. Soc., 2011, 133, 9228; (h) Y. Lu, S. K. Woo and M. J. Krische, J. Am. Chem. Soc., 2011, 133, 13876; (i) Y. Zhang, Q. Guo, X. Sun, J. Lu, Y. Cao, Q. Pu, Z. Chu, L. Gao and Z. Song, Angew. Chem. Int. Edn., 2018, 57, 942.

6. P. A. Wender, C. T. Hardman, S. Ho, M. S. Jeffreys, J. K. Maclaren, R. V. Quiroz, S. M. Ryckbosch, A. J. Shimizu, J. L. Sloane and M. C. Stevens, Science, 2017, 358. 218.

7. M. G. Kazanietz, N. E. Lewin, F. Gao, G. R. Pettit and P. M. Blumberg, Mol. Pharmacol., 1994, 46, 374.

8. P. A. Wender, C. M. Cribbs, K. F. Koehler, N. A. Sharkey, C. L. Herald, Y. Kamano, G. R. Pettit and P. M. Blumberg, Proc. Natl. Acad. Sci. USA., 1988, 85, 7197.

9. (a) P. A. Wender, J. L. Baryza, C. E. Bennett, F. C. Bi, S. E. Brenner, M. O. Clarke, J. C. Horan, C. Kan, E. Lacôte, B. Lippa, P. G. Nell and T. M. Turner, J. Am. Chem. Soc., 2002, 124, 13648; (b) P. A. Wender, B. A. Loy and A. J. Schrier, Isr. J. Chem., 2011, 51, 453; (c) P. A. Wender, J. L. Baryza, S. E. Brenner, B. A. DeChristopher, B. A. Loy, A. J. Schrier and V. A. Verma, Proc. Natl. Acad. Sci. USA., 2011, 108, 6721.

10. (a) G. E. Keck, W. Li, M. B. Kraft, N. Kedei, N. E. Lewin and P. M. Blumberg, Org. Lett., 2009, 11, 2277; (b) G. E. Keck, Y. B. Poudel, A. Rudra, J. C. Stephens, N. Kedei, N. E. Lewin, M. L. Peach and P. M. Blumberg, Angew. Chem. Int. Ed., 2010, 49, 4580; (c) G. E. Keck, Y. B. Poudel, A. Rudra, J. C. Stephens, N. Kedei, N. E. Lewin and P. M. Blumberg, Bioorg. Med. Chem. Lett., 2012, 22, 4084; (d) M. B. Kraft, Y. B. Poudel, N. Kedei, N. E. Lewin, M. L. Peach, P. M. Blumberg and G. E. Keck, J. Am. Chem. Soc., 2014, 136, 13202; (e) M. J. Krische, J. M. Ketcham, I. Volchkov, T.-Y. Chen, P. M. Blumberg, N. Kedei and N. E. Lewin, J. Am. Chem. Soc., 2016, 138, 13415.

11. (a) A. P. Green, S. Hardy, A. T. L. Lee and E. J. Thomas, Org. Biomol. Chem., 2017, 15, 9497; (b) A. P. Green, S. Hardy and E. J. Thomas, Org. Biomol. Chem., 2017, 15, 9475; (c) R. Dumeunier, T. Gregson, S. MacCormick, H. Omori and E. J. Thomas, Org. Biomol. Chem., 2017, 15, 2768; (d) M. Ball, A. Baron, B. Bradshaw, M. O'Brien, R. Dumeunier and E. J. Thomas, Org. Biomol. Chem., 2016, 14, 9650.

12. J. L. Roberts, J. Borgese, C. Chan, D. D. Keith and C.-C. Wei, Heterocycles, 1993, 35, 115.

13. (a) B. Linclau, A. J. Boydell, R. S. Timofte, K. J. Brown, V. Vinader and A. C. Weymouth-Wilson, Org. Biomol. Chem., 2009, 7, 803; (b) L. Bonnac, S. E. Lee, G. T. Giuffredi, L. M. Elphick, A. A. Anderson, E. S. Child, D. J. Mann and V. Gouverneur, Org. Biomol. Chem., 2010, 8, 1445.

14. B. A. Loy, A. B. Lesser, D. Staveness, K. L. Billingsley, L. Cegelski and P. A Wender, J. Am. Chem. Soc., 2015, 137, 3678.

15. P. R. Mears and E. J. Thomas, Tetrahedron Lett., 2015, 56, 3975.

16. P. R. Mears and E. J. Thomas, Tetrahedron Lett., 2015, 56, 3980.

17. (a) F. Olimpieri, S. Fustero, A. Volonterio and M. Zanda, Synthesis, 2010, 651;

18. J. M. Andrés, M. A. Martínez, P. Pedrosa and A. PérezEncabo, Synthesis, 1996, 1070.

19. S. Raghavan and K. V. Vinoth, Org. Biomol. Chem., 2013, 11, 2847. 
20. H. Sugiyama, F. Yokokawa and T. Shioiri, Tetrahedron, 2003 59, 6579.

21. C. Guo, X. Yue and F.-L. Qing, Synthesis, 2010, 1837.

22. (a) T. Taguchi, O. Kitagawa, T. Morikawa, T. Nishiwaki, H. Uehara, H. Endo and Y. Kobayashi, Tetrahedron Lett., 1986, 27, 6103; (b) K. Sato, M. Omote, A. Ando and I. Kumadaki, J. Fluorine Chem., 2004, 125, 509; (c) R. Surmont, G. Verniest, J. W. Thuring, G. Macdonald, F. Deroose and N. De Kimpe, J. Org. Chem., 2010, 75, 929; (d) K. Sato, R. Kawata, F. Ama, M. Omote, A. Ando and I Kumadaki, Chem.\& Pharm. Bull., 1999, 47, 1013.

23. (a) H. Wang, Y. Zhao, Y. Li and L. Lu, J. Org. Chem., 2006, 71, 3278; (b) T. Konno, T. Takehana, M. Mishima and T. Ishihara, J. Org. Chem., 2006, 71, 3545; (c) V. Bizet, X. Pennecoucke, J.-L. Renard and D. Cahard, J. Fluorine Chem., 2013, 152, 56; (d) J. A. Codelli, J. M. Baskin, N. Agard and C. R. Bertozzi, J. Am. Chem. Soc., 2008, 130, 11486; (e) N. Shimojuh, Y. Imura, K. Moriyama and H. Togo, Tetrahedron, 2011, 67, 951; (f) H. Nemoto, A. Satoh, K. Fukumoto and C. Kabuto, J. Org. Chem., 1995, 60, 594.

24. J.-S. Zheng, H.-N. Chang, F.-L. Wang and L. Liu, J. Am. Chem. Soc., 2011, 133, 11080.

25. (a) Z.-Y. Yang and D. J. Burton, J. Org. Chem., 1991, 56, 1037; (b) D. Seyferth, R. M. Simon, D. J. Sepelak and H. A. Klein, J. Org. Chem., 1980, 45, 2273; (c) D. Seyferth and K. R. Wursthorn, J. Organomet. Chem., 1979, 182, 455; (d) D. Seyferth, R. M. Simon, D. J. Sepelak and H. A. Klein, J. Am. Chem. Soc., 1983, 105, 4634; (e) Z.-Y. Yang and D. J. Burton, J. Fluorine Chem., 1989, 44, 339; (f) M. Kirihara, T. Takuwa, S. Takizawa and T. Momose, Tetrahedron Lett., 1997, 38, 2853; (g) M. Kirihara, T. Takuwa, S. Takizawa, T. Momose and H. Nemoto, Tetrahedron, 2000, 56, 8275.

26. (a) G. H. Posner, H. J. Kim, M. Kahraman, H. B. Jeon, B. C. Suh, H. Li, P. Dolan and T. W. Kensler, Bioorg. Med. Chem., 2005, 13, 5569; (b) X. Creary, J. Org. Chem., 1987, 52, 5020.

27. Q-Q. Min, Z. Yin, Z. Feng, W.-H. Guo and X. Zhang, J. Am. Chem. Soc., 2014, 136, 1230.

28. E. Van Hende, G. Verniest, R. Surmont and N. De Kimpe, Org. Lett., 2007, 9, 2935.

29. J. M. Concellón, H. Cuervo and R. Fernández-Fano, Tetrahedron, 2001, 57, 8983.

30. T. Eguchi, T. Aoyama and K. Kakinuma, Tetrahedron Lett., 1992, 33, 5545.

31. J. Fried and E. A. Hallinan, Tetrahedron Lett., 1984, 25, 2301.

32. (a) H. Wang, Y. Zhao, Y. Li and L. Lu, J. Org. Chem., 2006, 71, 3278; (b) X.-H. Xu, X.-L. Qiu, X. Zhang and F.-L. Qing, J. Org. Chem., 2006, 71, 2820.

33. (a) B. Plietker and M. Niggemann, Org. Lett., 2003, 5, 3353; (b) B. Plietker, Synthesis, 2005, 15, 2453; (c) A. P. Massey, V. S. Pore and B. G. Hazra, Synthesis, 2003, 3, 426.

34. (a) Y. Ding and G. Zhao, Tetrahedron Lett., 1992, 33, 8117; (b) L. M. Fleury, A D. Kosal, J. T. Masters and B. L. Ashfeld, J. Org. Chem., 2013, 78, 253.

35. M. Ball, T. Gregson, H. Omori and E. J. Thomas, Org. Biomol. Chem., 2017, 15, 2740.

36. P. A. Wender, Y. Nakagawa, K. E. Near and D. Staveness, Org. Lett. 2014, 16, 5136.
37. P. A. Wender and D. Staveness, Org. Lett. 2014, 16, 5140.

38. H. Zhang, S. Neimanis, L. A. Lopez-Garcia, J. M. Arenciba, S. Amon, A. Stroba, S. Zeuzem, E. Proschak, H. Stark, A. F. Bauer, K. Busschots, K. Busschots, T. J. Jorgensen, M. Engel, J. O. Schulze and R. M. Biondi, Chem. Biol., 2014, 21, 754.

39. A. F. Rudolf, T. Skovgaard, S. Knapp, L. J. Jensen and Berthelsen, PloS One, 2014, 9, 98800.

40. D. M. Foulkes, D. P. Byrne, W. Yeung, S. Shrestha, F. P. Bailey, S. Ferries, C. E. Eyers, K. Keeshan, C. Wells, D. H. Drewry, W. J. Zuercher, N. Kannan and P. A. Eyers, Sci. Signal, 2018, 11, 7951.

41. D. P. Byrne, M. Vonderach, S. Ferries, P. J. Brownridge, C. E. Eyers and P. A. Eyers, Biochem. J., 2016, 473, 3159.

42. H. W. Lee, L. Smith, G. R. Pettit and J. B. Smith, Mol. Pharmacol., 1997, 51, 439.

43. D. R. Alessi, FEBS Lett., 1997, 402, 121.

44. J. M. Murphy, Q. Zhang, S. N. Young, M. L. Reese, F. P. Bailey, P. A. Eyers, D. Ungureanu, H. Hammaren, O. Silvennoinen, L. N. Varghese, K. Chen, A. Tripaydonis, N. Jura, K. Fukuda, J. Qin, Z. Nimchuk, M. B. Mudgett, S. Olowe, C. L. Gee, L. Liu, R. J. Daly, G. Manning, J. J. Babon, and I. S. Lucet, Biochem. J., 2014, 457, 323. 